\title{
EARLY WILDFIRE DETECTION USING TEMPORAL FILTERING AND MULTI-BAND INFRARED ANALYSIS
}

\author{
A Thesis \\ presented to \\ the Faculty of California Polytechnic State University, \\ San Luis Obispo
}

\author{
In Partial Fulfillment \\ of the Requirements for the Degree \\ Master of Science in Engineering
}

by

Ansel John Boynton

June, 2013 
(C) 2013

Ansel John Boynton

\section{ALL RIGHTS RESERVED}

Page ii 
COMMITTEE MEMBERSHIP

TITLE:

AUTHOR:

DATE SUBMITTED:

COMMITTEE CHAIR:

COMMITTEE MEMBER:

COMMITTEE MEMBER:
Early Wildfire Detection Using Temporal Filtering and Multi-Band Infrared Analysis

Ansel John Boynton

June, 2013

Dr. John Saghri, Professor

Electrical Engineering Department

Dr. John Jacobs, Raytheon Professor of Practice, Electrical Engineering Department

Dr. Jane Zhang, Associate Professor, Electrical Engineering Department 


\begin{abstract}
Early Wildfire Detection using Temporal Filtering and Multi Band Infrared Analysis Ansel John Boynton
\end{abstract}

Every year wildfires threaten or destroy ecological habitats, man-made infrastructure and people's lives. Additionally millions of dollars are spent each year trying to prevent and control these fires. Ideally if a wildfire can be detected before it rages out of control it can be extinguished and avoid large scale devastation. Traditional manned fire lookout towers are neither cost effective nor particularly efficient at detecting wildfire. It is proposed that temporal filtering can be used to isolate the signals created at the beginnings of potential wildfires. Temporal filtering can remove any background image and any periodic signals created by the camera movement. Once typical signals are analyzed, digital filters can be designed to pass fire signals while blocking the unwanted signals. The temporal filter passes only fire signals and signals generated by moving objects. These objects can be distinguished from each other by analyzing the objects mid and long wave energy profile. This algorithm is tested on 17 data sources and its results analyzed.

Keywords: Forest Fires, Digital Image Processing, Digital Signal Processing, Cooled Mid Wave Infrared, Cooled Long Wave Infrared 


\section{ACKNOWLEDGMENTS}

I acknowledge the following people for their efforts and contributions to my thesis. It would not have been completed without them.

Special thanks to my thesis advisors, Dr. John Saghri and Dr. John Jacobs, for their guidance, patience, support and encouragement in my graduate study.

Additional thanks for Dr. Jane Zhang for agreeing to be on my committee

I thank Raytheon Employees: Steve Botts, Marc Bauer, Chris Tracy, and Dana Schneider for their support during the June 2011 Fire Test as well as Gary Hughes from FLIR for his help with IR cameras.

I thank all the previous students who worked on this project: Ryan Radjabi, Timothy Davenport, Daniel Kohler, George Moussa and Alex Aldama. This thesis could not be completed without the foundation they built.

I thank my supervisors and co-workers who pushed me to pursue my educational goals while in the Air Force. Without them I would not be in this position today.

I thank my parents, sister and friends for their encouragement and support in helping me complete my thesis. 


\section{Contents}

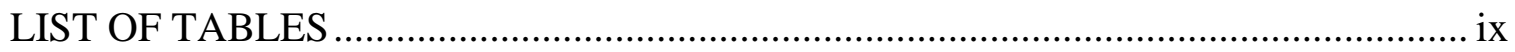

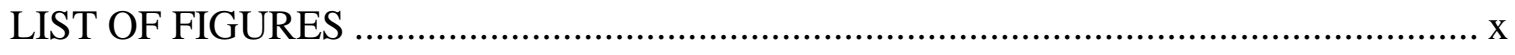

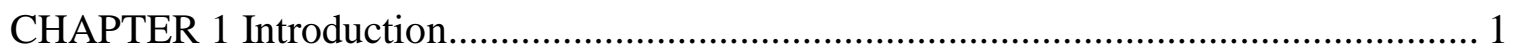

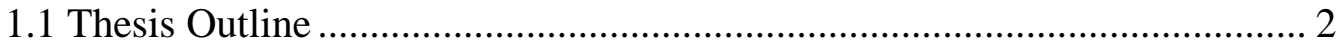

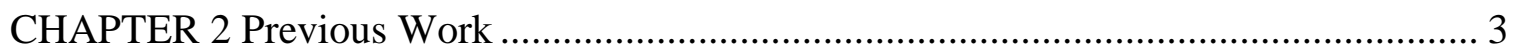

2.1 Current Implementations ................................................................... 3

2.2 Research preformed at Cal Poly ................................................................. 4

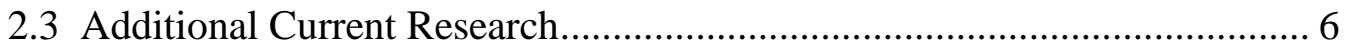

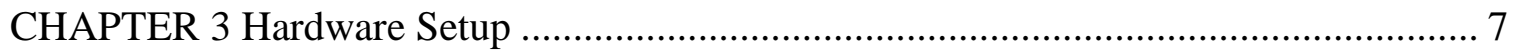

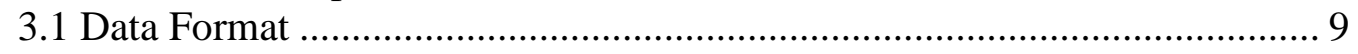

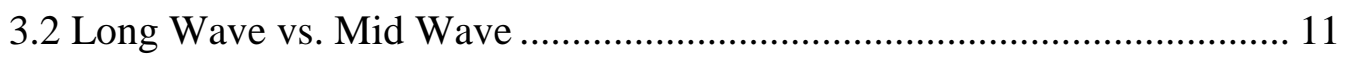

CHAPTER 4 Frequency Analysis of Various Signals.................................................... 14

4.1 Saturated Fire signal ............................................................................. 15

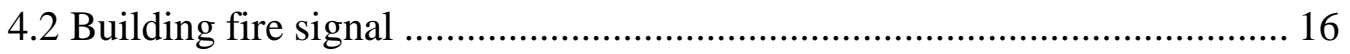

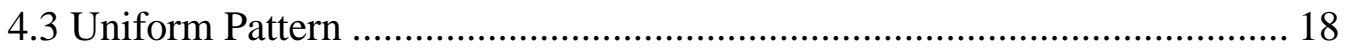

4.4 Textured Pixel Signal........................................................................ 20

4.5 Strong Edge Signal ................................................................................. 21

Page vi 
4.6 Moving Car Pixel

4.7 Conclusion of Frequency Analysis

CHAPTER 5 Principals Used in Design of Fire Detection ........................................ 26

5.1 FIR High Pass Filter Design ............................................................. 26

5.1.1 Creation of a Low Pass Prototype Filter ........................................... 26

5.1.2 Window Low Pass Prototype .......................................................... 27

5.1.3 Convert Low Pass Prototype to a High Pass Filter .............................. 27

5.2 IIR Filter Band Pass Design ......................................................... 28

5.2.1 Create Analog Low Pass Prototype .................................................. 28

5.2.2 Convert Analog Low Pass to Analog Band Pass .................................. 29

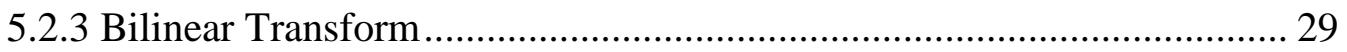

5.3 Gaussian Blurring Spatial Filter........................................................ 30

5.4 Connected Component Analysis ..................................................... 31

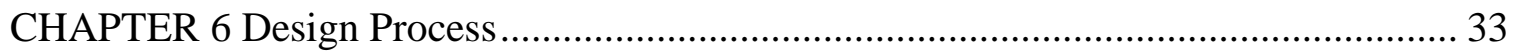

6.1 FIR High Pass Filter Implementation ................................................. 33

6.2 IIR Band Pass filter Implementation.................................................... 36

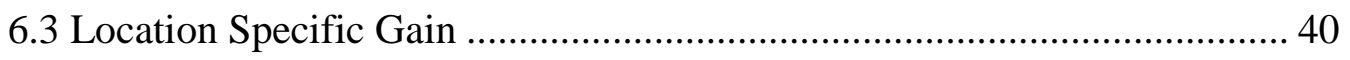

6.4 Spatial Filter and Connected Component Analysis ................................ 43 
6.5 Fire Detection by Features ......................................................................... 44

6.6 Time Averaging Filtering ……………………………......................... 47

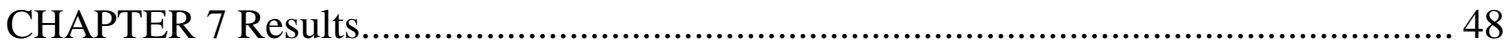

7.1 Charcoal1 Results ......................................................................... 50

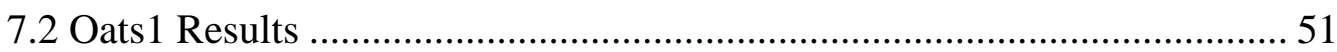

7.3 Examining False Positives ..................................................................... 52

7.3.1 Initial Movement Frames ..................................................................... 54

7.3.2 High Altitude False Positive ................................................................ 54

7.3.3 False Positive due to Street Light ........................................................ 55

CHAPTER 8 Proposed Implementation ...................................................................... 57

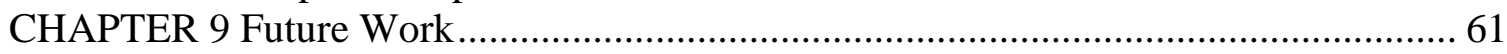

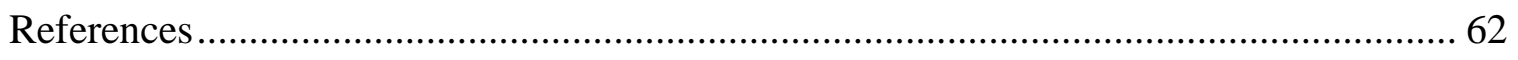

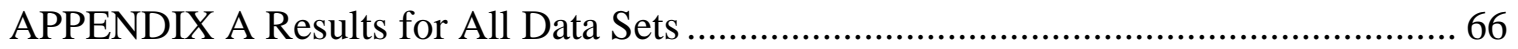

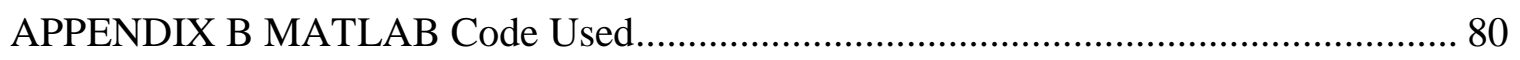




\section{LIST OF TABLES}

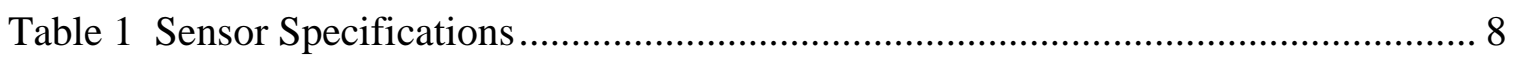

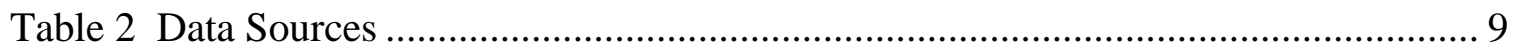

Table 3 Window Function Characteristics [20] ................................................... 27

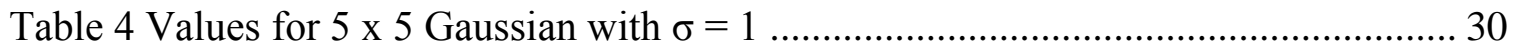

Table 5 FIR High Pass Filter Legend .................................................................. 34

Table 6 Performance of IIR Band Pass Filters......................................................... 38

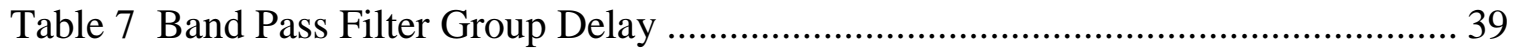

Table 8 Total Number of False Positives for Each Data Set ..................................... 53

Table 9 Algorithm Processing Time Requirements .................................................. 59

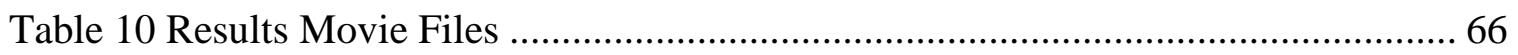

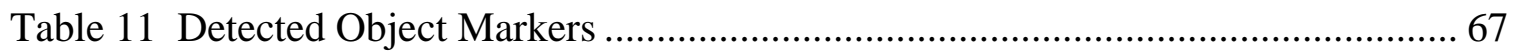




\section{LIST OF FIGURES}

Figure 1 Satellite Image of 2007 San Diego Wildfires ............................................... 1

Figure 2 Sensor Mast Configuration................................................................... 7

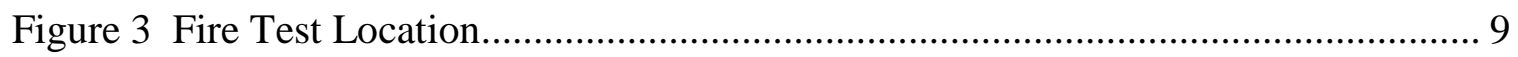

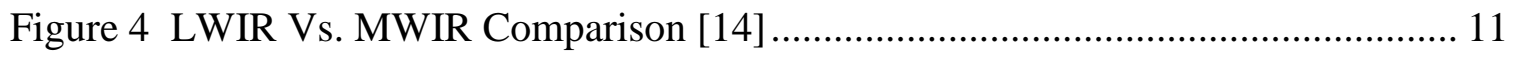

Figure 5 Spectral Energy form a Forest Fire [16] ................................................ 12

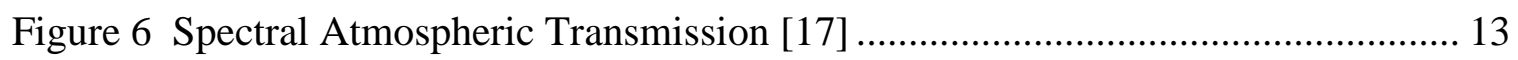

Figure 7 MWIR and LWIR Intensities for Center of Fire in Oats1 Data Set ................. 15

Figure 8 Temporal and Frequency Profile of a Saturated Fire Pixel ........................... 16

Figure 9 Temporal and Frequency Profile of Unsaturated Center Fire Pixel

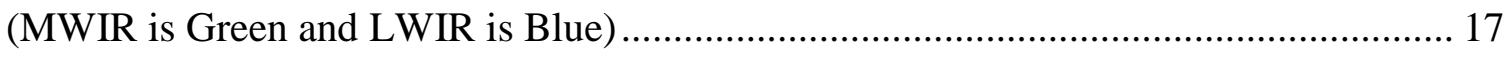

Figure 10 Frequency Profile of Pixels Moving Away From Center of Fire

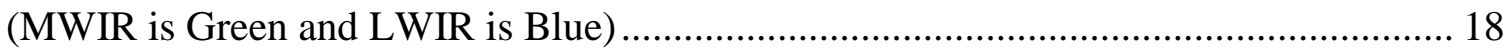

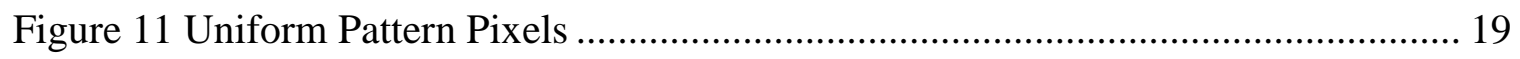

Figure 12 Frequency Profile of Uniform Location ................................................ 19

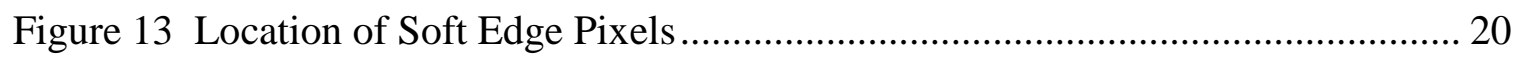

Figure 14 Frequency Profile of Textured Signals

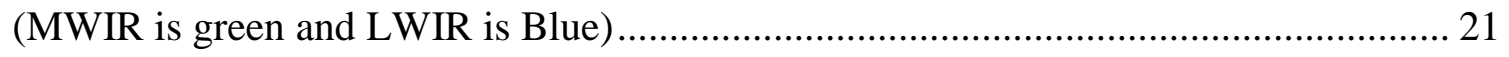

Figure 15 Location of Selected Strong Edge Pixels ................................................ 22

Figure 16 Frequency Profile of Strong Edge Signals

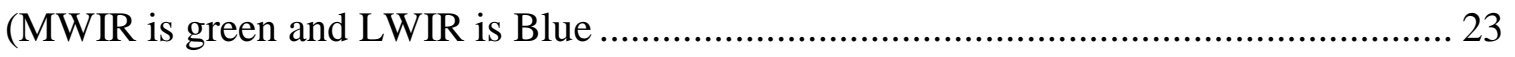


Figure 17 Frequency Profile of Moving Object Signals

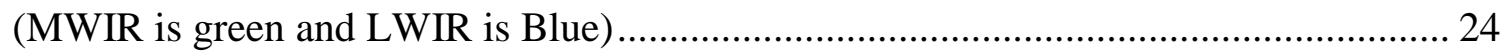

Figure 18 Location of Proposed Digital Filter Pass Bands ........................................ 25

Figure 19 FIR High Pass Filter Design Procedure ................................................... 26

Figure 20 IIR Band Pass Design Procedure ............................................................ 28

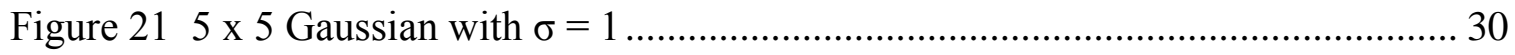

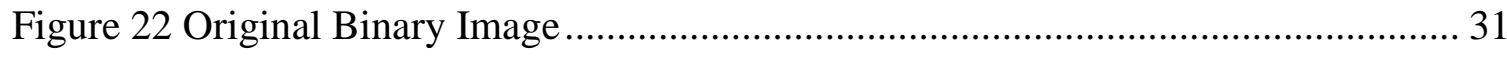

Figure 23 Results of First Pass and Final Results of Connected Component Analysis... 32

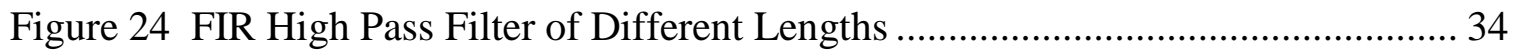

Figure 25 Final FIR High Pass Filter Desgin..................................................... 35

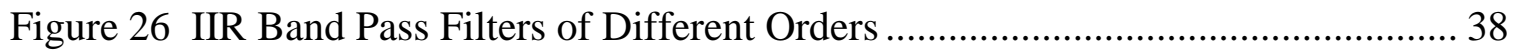

Figure 27 Group Delay of IIR Band Pass Filters .................................................. 39

Figure 28 Final Design of IIR Band Pass Filter...................................................... 40

Figure 29 Expected Values for frequencies in the Band Pass Region for

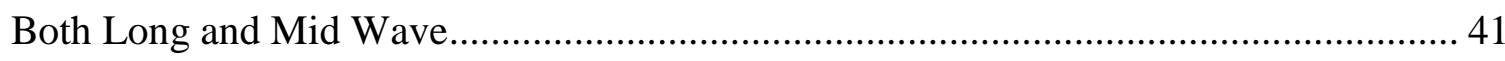

Figure 30 Corrected Values for Both Long and Mid Wave...................................... 42

Figure 31 Pixel Specific Gain for both Long and Mid Wave band pass filter................ 43

Figure 32 High Pass Original Image, Blurred Image and Binary Image ....................... 44

Figure 33 Decision Tree for Detected Objects ................................................. 44

Figure 34 Moving Object (High Pass on Right Band Pass on Left) ............................ 45

Figure 35 Long Wave vs. Mid Wave Power of a Small Fire....................................... 46

Figure 36 Long Wave Vs. Mid Wave Power of a Moving Object .............................. 47 
Figure 37 Strong Edge Frequencies for Stable Sample Rate (Left)

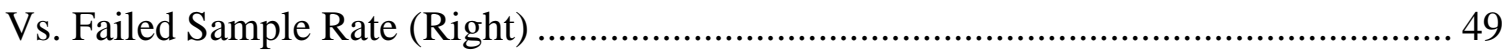

Figure 38 Results due to Failed Frame Rate .............................................................. 50

Figure 39 Charcoal1 Results Band Pass is Green and High Pass is Blue ........................ 51

Figure 40 Oats1 Results, Band Pass is Green and High Pass is Blue .............................. 52

Figure 41 Example of false positive due to movement.................................................. 54

Figure 42 False Positive due to High Altitude Source.................................................... 55

Figure 43 False Positive due to Street Light ................................................................ 56

Figure 44 Algorithm Block Diagram ..................................................................... 58 


\section{CHAPTER 1}

Introduction

Every year there thousands of wildfires cause damage across the United States. The United States spend on average 900 million dollars a year on fighting wildfires []. Due to southern California's hot dry summer it is especially vulnerable. According to Cal Fire in 2011 California alone there were 3,056 wildfires burning 51,889 acres of land costing the state of 100 million dollars [1]. The five largest California wildfires by acre took place in the southern half of the state. While most of these were away from populated areas it is not unheard of for a wildfire to cause significant damage to populated areas. The wildfires of late October of 2007 destroyed over 2,000 structures in San Diego County during the last quarter of 2007 [2]. Because of the fire's large size satellites in space could easily identify them. Figure 1 shows the progress of the San Diego fires on October 23, 2007 [3].

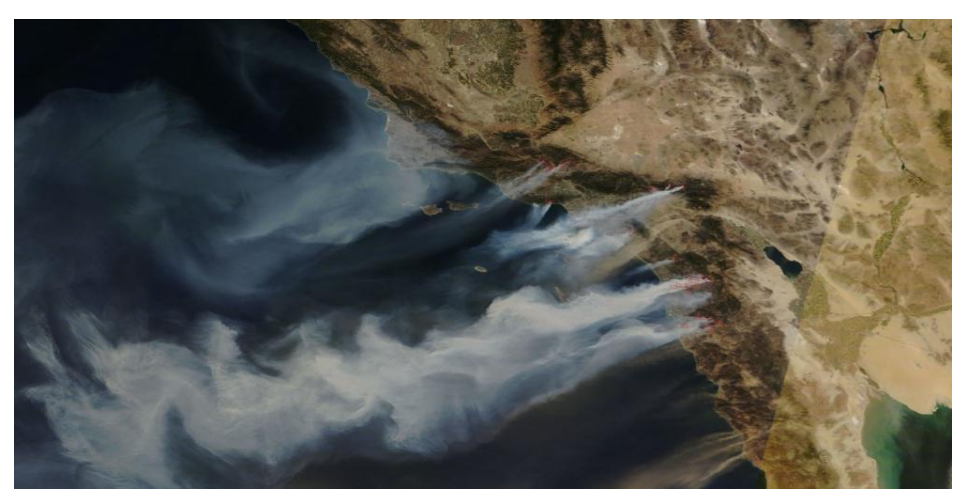

Figure 1 Satellite Image of 2007 San Diego Wildfires

A more cost effective way of fighting fires is to extinguish a small fire before it grows into a large one. If a fire is detected early enough, lives and property can be saved 
at a reduced cost. The goal of this project is to automatically detect fires remotely and as small as possible so fire fighters can extinguish them before they grow out of control.

\subsection{Thesis Outline}

This thesis is structured as follows. Chapter 2 discusses previous work performed both at Cal Poly and elsewhere implemented solutions. Chapter 3 discusses the hardware used to capture data throughout the test fires. Chapter 4 analyzes the data captured during the burns. Chapter 5 covers the theory behind the proposed solution. Chapter 6 follows the design process using the theory explained in chapter 5. Chapter 7 analyzes the recorded results from the test burns using the proposed algorithm. Chapter 8 briefly discusses possible implementations of the system. Finally Chapter 9 looks at future work. 


\section{CHAPTER 2}

Previous Work

There has been plenty of work done in the automatic detection of fires in recent years. Current implementations range from small scale, an indoor smoke detector to large scale, detection using satellite imagery. At Cal Poly forest fire detection has been an ongoing project mostly focused on daytime solutions identifying smoke. Finally Yasar Sahin discusses a very different proposed solution using active radar [5].

\subsection{Current Implementations}

The most traditional implementation of detecting forest fires is the manned fire watch tower. These towers are located in remote areas with a high fire risk. An individual watches the surrounding area looking for smoke from the start of a fire. This is an expensive and ineffective solution. Advanced sensors and computer vision algorithms can reduce cost and increase effectiveness.

The most common form of fire detection is the conventional smoke detector. These detectors introduced in the late 70 s are now in $95 \%$ percent of all homes. These detectors work by ionized particles from the smoke breaking an internal beam. These are very effective in internal home fires and Cal Fire states a working smoke detector improves a person's survivability by 50 percent [6]. These detectors only work in an enclosed space close to the fire. They are not a practical solution for identifying fires in large open areas. 
Multiple countries have optical based fire detection systems. Germany, South Africa, Canada, England and France all use systems based on detecting smoke in the visible range during the day and infrared data at night [7]. These systems generate many false positives due the atmospheric events and human disturbances. Constant human interaction is required the verify fire conditions. The desired system contains increased fire signals and fewer false positives.

Satellite imagery is probably the largest scale of all the fire detection techniques [8]. One of the satellites NASA current uses the TERRA and AQUA equipped with the MODIS (Moderate Resolution Imaging Spectroradiometer) for multi spectral analysis. Equipped to analyze 36 bands the MODIS sensor's supports a maximum ground resolution of 250 meters [9]. While this can be used to detect and track large fires, it does not provide high enough resolution to detect smaller fires. The fire generated in this testing never grew over 1 one squared meter and would never register on the MODIS sensor. Additionally these satellites are constantly in motion. It takes one to two days before the satellite can return the same spot. A fire can easily grow out of control between visits.

\subsection{Research preformed at Cal Poly}

Early fire detection is an ongoing project at Cal poly. Three previous theses designed algorithms to detect the presence of a forest fire. For the most part these efforts focused on detecting the smoke plume generated by the fire. Typically a smoke plume covers a much larger area than the actual heat of the fire. 
In 2012 Timothy Davenport proposed on texture analysis of data put through a principal component analysis (PCA) to detect smoke from a fire [10]. A PCA is performed on images from red, blue, green, mid wave infrared and long wave infrared frames. Frames from two different times are taken per band for a total of 10 images. The smoke then appears on one of the higher order principal components. Texture analysis is performed via Gray Level Co-Occurrence Matrix. The principal component containing the smoke will contain noise everywhere except the smoke plume and this smoke plume can then be extracted.

At the same time Daniel Kohler produced his results on just identifying a fire off cooled mid wave infrared data [11]. Kohler proposed to classify the edge of the fire by mean, variance and principal component metrics as the fire expanded and contracted. These changes due to the fire's growth increase the number of outlying values along the edge of the fire which can be identified by comparing a pixels variance over time to its medium or mean. The effect of the outliers also appears in the principal component analysis of the frames over time. This method is unique as it is the first method studied at Cal Poly which uses only infrared data and may work for 24 hour coverage.

Later in 2012 George Moussa proposed a different take in using a principal component analysis [12]. In Moussa's approach a principal component analysis was performed on the blue image over nine different time frames. The smoke plume is then extracted by using a combination of blurring, median filtering, adaptive thresholding and Gaussian mixture model. 
Alex Aldama is concurrently working on the same project fire detection project. His proposal also aims towards a 24 hour solution using mid and long wave infrared data. His proposal uses a neural network to classify pixels based on the difference in magnitude of the mid and long wave images. He also uses statistical information of each possible fire pixel's neighborhood to correctly classify the pixel.

\subsection{Additional Current Research}

Additional research has been done on an alternative form of fire detection. Instead of looking for the heat or smoke of the fire, the process explained in Early Forest Fire Detection Using Radio-Acoustic Sounding System [5] uses the change in frequency of a sound wave to detect heated air. As a sound wave travels through the air its frequency changes if the air temperature increases. A radar system can then identify this change in frequency. Of all the systems discussed this is the only active system. The radar component adds considerable cost to the system. The simulated results look to identify a fire with a resolution of 25 square meters. This is still much larger than the fires identified in this test but a much smaller fire than satellite imagery could ever detect. 


\section{CHAPTER 3}

\section{Hardware Setup}

The data used for this analysis was captured on June 18th, 2011 at Raytheon Vision Systems (RVS) in Goleta, California. Data was captured using four different cameras: a visible light camera, a cooled mid-wave IR camera (CMWIR), a cooled long-wave IR (CLWIR) camera and an uncooled long-wave IR camera (UCLWIR). Figure 2 shows the configuration of the cameras on the mast.
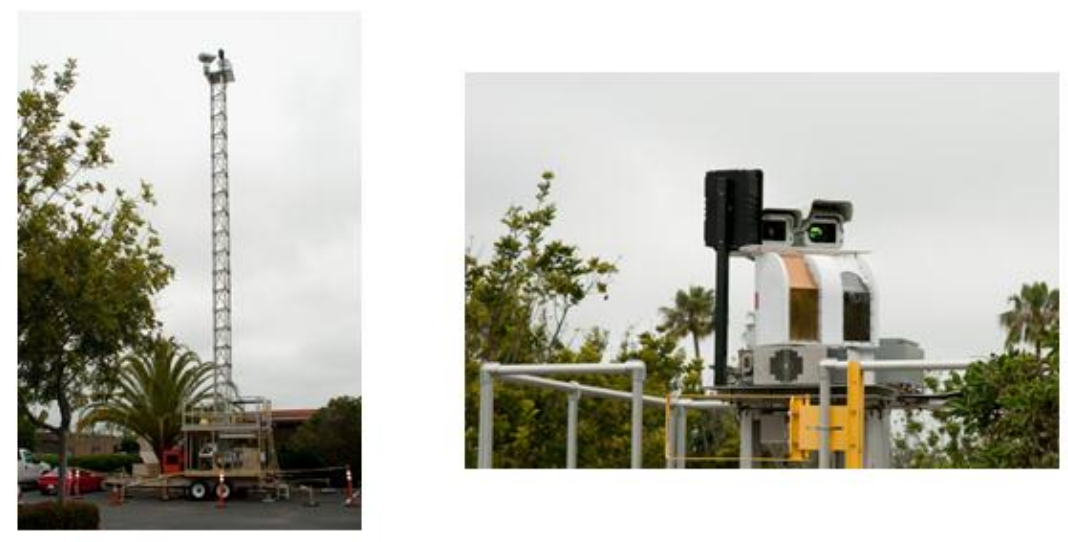

Figure 2 Sensor Mast Configuration

This thesis aims to work towards a solution which can be implemented during both the day and night so only the CMWIR camera and CLWIR camera were used. The visible light camera is not sensitive enough during the low light conditions of night and the UCLWIR showed no advantages versus the cooled long-wave camera. Additionally the cooled mid wave and long wave were collocated so their pixels line up one for one; no additional registration is required to align frames. Table 1 below shows the specifications of the CMWIR and CLWIR sensors. Because the uncooled long wave 
infrared source is never used, for the rest of this thesis mid wave or MWIR refers to the cooled mid wave infrared source and long wave or LWIR refers to the cooled long wave infrared source.

Table 1 Sensor Specifications

\begin{tabular}{|l|l|l|}
\hline Band & CMWIR & CLWIR \\
\hline Sensor Resolution & $640 \times 480$ & $640 \times 480$ \\
\hline Pixel Size & $20 \mu$ & $20 \mu$ \\
\hline Focal length & $100 \mathrm{~mm}$ & $100 \mathrm{~mm}$ \\
\hline Aperture & F2.2 & F2.3 \\
\hline Spectral Band & $3.8-5 \mu$ & $8-12 \mu$ \\
\hline Bits & 14 & 14 \\
\hline Technology & HgCdTe & HgCdTe \\
\hline Frames Per Second & 30 & 30 \\
\hline
\end{tabular}

All four cameras were mounted on a tower 50 feet high and 875 yards $(0.8 \mathrm{~km})$ away from the source of the fire. Figure 3 shows the positioning of the camera mast and fire location with the locations surroundings. The map was downloaded from Google Earth. 


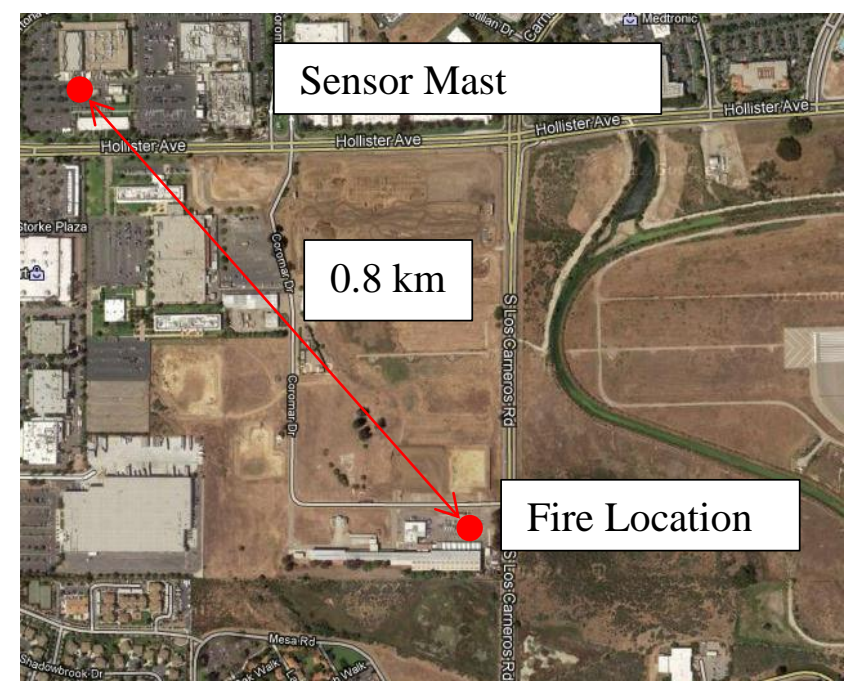

Figure 3 Fire Test Location

\subsection{Data Format}

A total of 17 recordings were taken in both MWIR and LWIR on June 17, 2011.

Table 1 shows all the different data sets in the order they were taken. The MWIR and LWIR data is combined in one raw file. These raw files were previously broken down into individual frames [10]. For the rest of this thesis all data generated from one raw file is considered a data set.

Table 2 Data Sources

\begin{tabular}{|c|c|c|}
\hline Data Set & Raw Movie File & $\begin{array}{l}\text { Number of } \\
\text { Frames }\end{array}$ \\
\hline Setup1 & $\begin{array}{l}\text { '2011-06-18_101158_LW-MW- } \\
\text { 14bit_setup.raw' }\end{array}$ & 3753 \\
\hline Charcoall & $\begin{array}{l}\text { '2011-06-18_102125_LW-MW- } \\
\text { 14bit_charcoal1.raw' }\end{array}$ & 4000 \\
\hline Oats1 & $\begin{array}{l}\text { '2011-06-18_102454_LW-MW- } \\
\text { 14bit_oats1.raw' }\end{array}$ & 8000 \\
\hline Oats2 & '2011-06-18_102925_LW-MW- & 395 \\
\hline
\end{tabular}

Page 9 


\begin{tabular}{|c|c|c|}
\hline & 14bit_oats2.raw' & \\
\hline Oats2b & $\begin{array}{l}\text { '2011-06-18_102949_LW-MW- } \\
\text { 14bit_oats2.raw' }\end{array}$ & 8000 \\
\hline Oats3 & $\begin{array}{l}\text { '2011-06-18_103820_LW-MW- } \\
\text { 14bit_oats3.raw' }\end{array}$ & 8000 \\
\hline Pine_needles & $\begin{array}{l}\text { '2011-06-18_104857_LW-MW- } \\
\text { l4bit pine needles.raw' }\end{array}$ & 14934 \\
\hline Pine_cones & $\begin{array}{l}\text { '2011-06-18_105906_LW-MW- } \\
\text { 14bit_pine_cones.râ'' }\end{array}$ & 8000 \\
\hline Palm & $\begin{array}{l}\text { '2011-06-18_110557_LW-MW- } \\
\text { 14bit_palm.raw' }\end{array}$ & 8000 \\
\hline Thistle & $\begin{array}{l}\text { '2011-06-18_110912_LW-MW- } \\
\text { 14bit_thistle.raw' }\end{array}$ & 6670 \\
\hline Wet_leaves & $\begin{array}{l}\text { '2011-06-18_111644_LW-MW- } \\
\text { 14bit_wet_leaves.raw' }\end{array}$ & 6063 \\
\hline Wet_dry1 & $\begin{array}{l}\text { '2011-06-18_112306_LW-MW- } \\
\text { 14bit_wet_dry1.raw' }\end{array}$ & 6117 \\
\hline Wet_dry2 & $\begin{array}{l}\text { '2011-06-18_112757 LW-MW- } \\
\text { 14bit_wet_dry2.raw' }\end{array}$ & 6364 \\
\hline Rosemary & $\begin{array}{l}\text { '2011-06-18_113916_LW-MW- } \\
\text { 14bit_rosemary.raw' }\end{array}$ & 5609 \\
\hline Applewood1 & $\begin{array}{l}\text { '2011-06-18_114605_LW-MW- } \\
\text { 14bit_applewood1.raw' }\end{array}$ & 6100 \\
\hline Applewood2 & $\begin{array}{l}\text { '2011-06-18_115209_LW-MW- } \\
\text { 14bit_applewood2.raw' }\end{array}$ & 6100 \\
\hline Extinguish & $\begin{array}{l}\text { '2011-06-18_121233_LW-MW- } \\
\text { 14bit_extinguish.raw' }\end{array}$ & 6100 \\
\hline
\end{tabular}

Each raw movie file was broken down into one raw image file for each MWIR and LWIR frame. Each MIWIR and LWIR frame held 14 bits of infrared data in a 16 bit frame. Humans require about 6 bits of grey information for an image to appear smooth while 8 bits of grey information is considered a high quality image [13]. The human eye cannot detect small changes in 14 bit information. This expanded bit depth can detect 
small changes that people cannot perceive. These small changes can lead to frequencies that would go undetected in a lower bit depth system.

\subsection{Long Wave vs. Mid Wave}

The two data sources used in this algorithm is the mid wave infrared and long wave infrared. Both data sets were taken from the same camera so the pixels line up one for one between the two data sources. Both bands can be used to remotely detect fire but both do this in a slightly different way. LWIR does a better job of directly measuring heat of the fire while MWIR energy is released from excited $\mathrm{CO}_{2}$ molecules. Heated $\mathrm{CO}_{2}$ is a product of any fire burning an organic fuel. Figure 4 shows a good example of the difference between a LWIR and MWIR image at the source of a fire, in the case of a butane cigarette lighter [14].

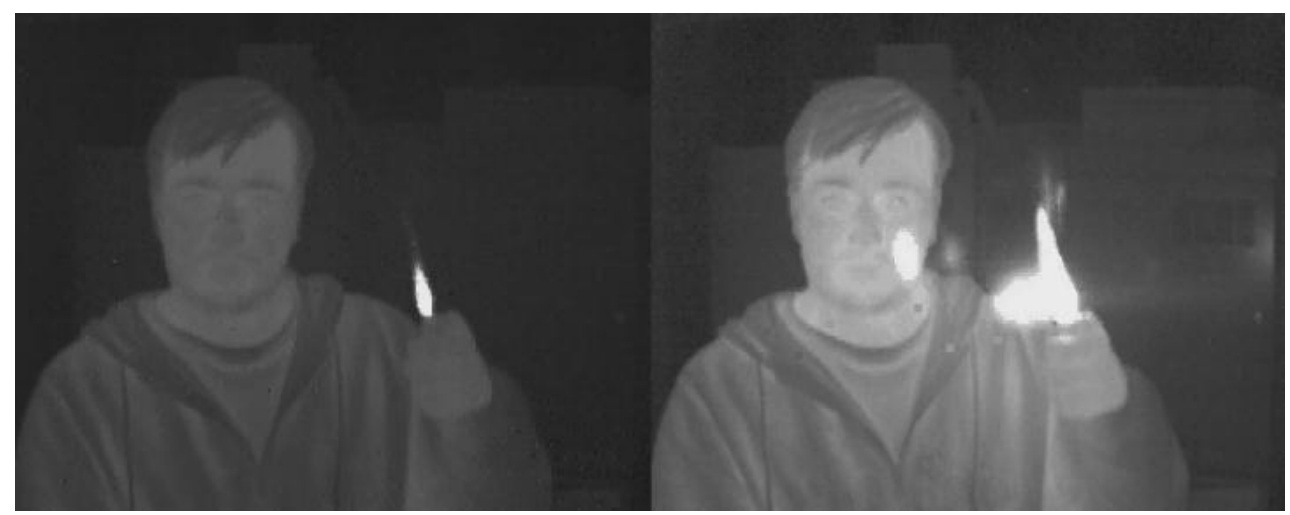

Figure 4 LWIR Vs. MWIR Comparison [14]

The image on the left, the LWIR image, shows a smaller but well defined hot spot at the center of the fire. The image on the right, the MWIR image, shows a larger but 
blurrier image of the fire. The fire has released excited $\mathrm{CO}_{2}$ which glows in the image. This heated air then acts as a light source illumination the person and his clothes behind him. The excited $\mathrm{CO}_{2}$ in the air also releases mid wave energy back to the sensor. This $\mathrm{CO}_{2}$ emission also plays a part in wildfire as $\mathrm{CO}_{2}$ is the largest chemical product of burning organic matter from a wild fire [15]. In 2006 a group led by Han Sun preformed a hyper spectral analysis using a land based and a satellite based sensor on an active forest fire to determine which spectral frequencies best identified a fire [16]. Figure 5 shows their results with the bandwidths of the MWIR and LWIR cameras used highlighted. The light grey line displays the fire measurements from a land sensor while the darker line displays the measurements from a sensor located on a satellite.

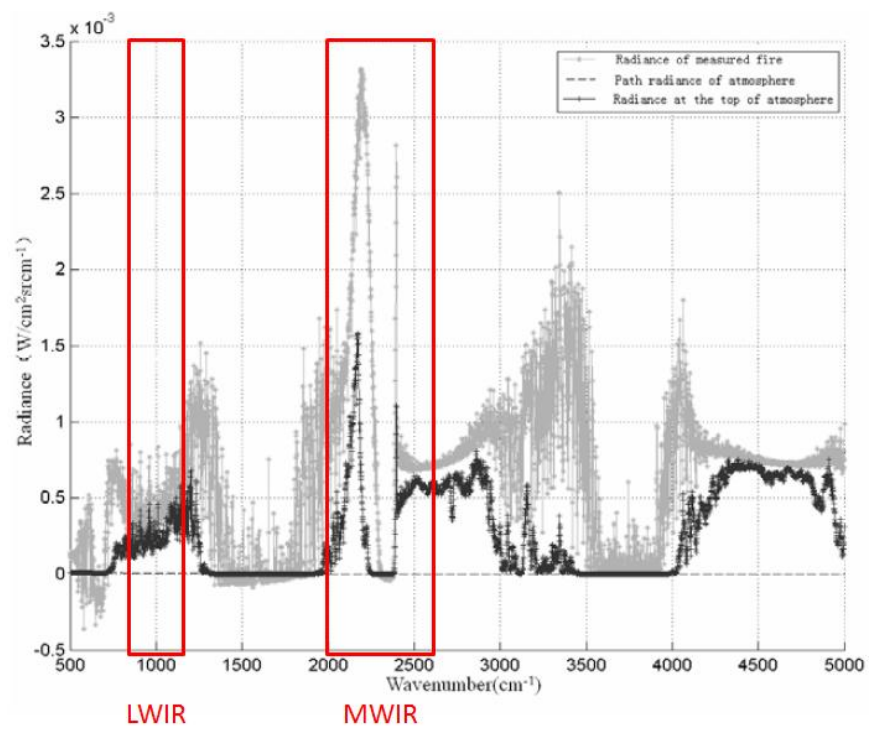

Figure 5 Spectral Energy form a Forest Fire [16]

In the case of a large forest fire the MWIR range appears to contain the most power but a large piece is missing from the center of the MWIR band. This experiment is not looking for a large fire but the beginnings of a small one. A small fire contains much 
less power in both ranges which causes the transmission medium to play a much larger role.

The $\mathrm{CO}_{2}$ in the air proves to be a larger factor as the camera is moved further and further away from the source. Figure 6 is a cutout of a larger Raytheon poster that shows how well the different bands transmit through the air [17]. The highlighted areas show the two data sources used.

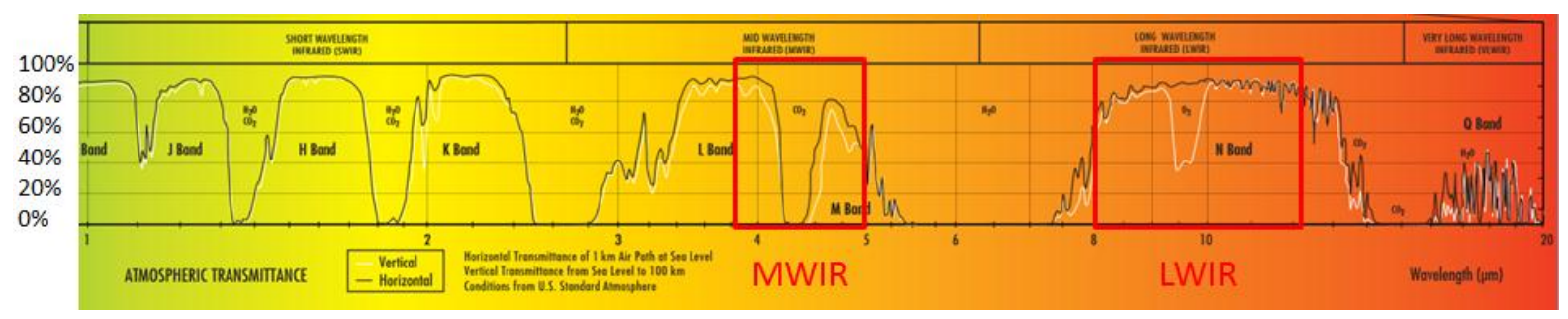

Figure 6 Spectral Atmospheric Transmission [17]

The black line shows the percent transmitted along the ground at sea level for 1 $\mathrm{km}$ or slightly further than our test range of $0.8 \mathrm{~km}$. At this distance almost $100 \%$ of the center MWIR energy gets absorbed by the atmosphere. This explains the missing piece for Figure 5. Once absorbed some of the energy of the MWIR signal will actually be retransmitted by the $\mathrm{CO}_{2}$ in the air. This retransmission causes the MWIR image to blur in both the spatial and temporal domains. The LWIR energy on the other hand receives around $90 \%$ of its original energy transmitted across its entire band. 


\section{CHAPTER 4}

Frequency Analysis of Various Signals

In order to understand which frequencies need to be passed or blocked various signals need to be analyzed. In this chapter the following signal types are analyzed: a building fire signal, a saturated fire signal, a strong edge signal, a uniform surface signal, a textured surface signal and a moving object signal. A textured surface is defined as a pixel whose neighbors have a small degree of intensity variation. In all cases the DC signals or unchanging image contains much more power than any frequency. Because of this every frequency plot cuts off the top of the DC power to display the frequency information with greater resolution. All signals except the fire signals and the moving object signals are analyzed over the entire 8000 frames of the oats 1 data set. Every pixel location is labeled by its row and column in parentheses in the following format: (row, column). The frequency magnitude display by the Discrete Fourier Transform (DFT) plot depends on the total number of samples so fire and moving object powers cannot be compared against the periodic signals analyzed over 8000 frames. The intensity value a pixel containing the desired signal is recorded for each frame of the data set. A DFT is performed on this recording to display the desired frequency information. When possible, more than one pixel is analyzed, multiple pixels' frequency responses are averaged together to create a better representation of the dominate frequency information. To start with we will investigate the two types of fire signals.

The fire signal can be broken into two different categories: a building fire signal and a saturated fire signal. Neither of these "fire" signals actually views the fire itself. 
The barbeque and a small hill block the line of sight from the fire to the sensor. The signals under analysis actually view the heat plume generated by the fire. Figure 7 below show the time based signal of the center of the heat plume, pixel $(367,351)$ of long wave data.
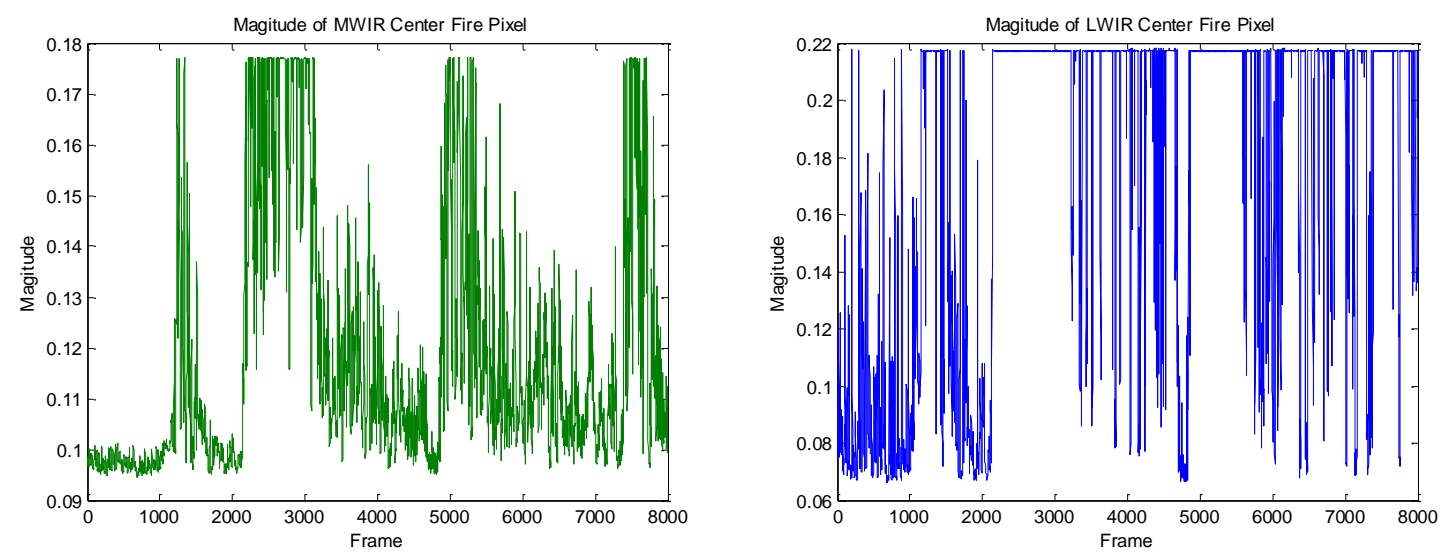

Figure 7 MWIR and LWIR Intensities for Center of Fire in Oats1 Data Set

It is noticed that for large sections of long wave band the data appears clipped. The mid wave shows similar clipping but for much shorter periods of time. These sections need analysis to see if the signals are in fact clipped by the sensor or if at some point the environment limits the intensity of the fire. When looking at the early stages of the fire signal, a building fire, frames 1 through 1085 show an unsaturated signal while frames 2400 through 3050 show a saturated fire signal.

\subsection{Saturated Fire signal}

The center fire signal's frequency was analyzed using a DFT from frames 2400 to 3050 in the long wave band. These 650 frames create the frequency spectrum shown in 
Figure 8. The results reveal that almost the entire signal is a constant value with nearly uniform power for all other frequencies.
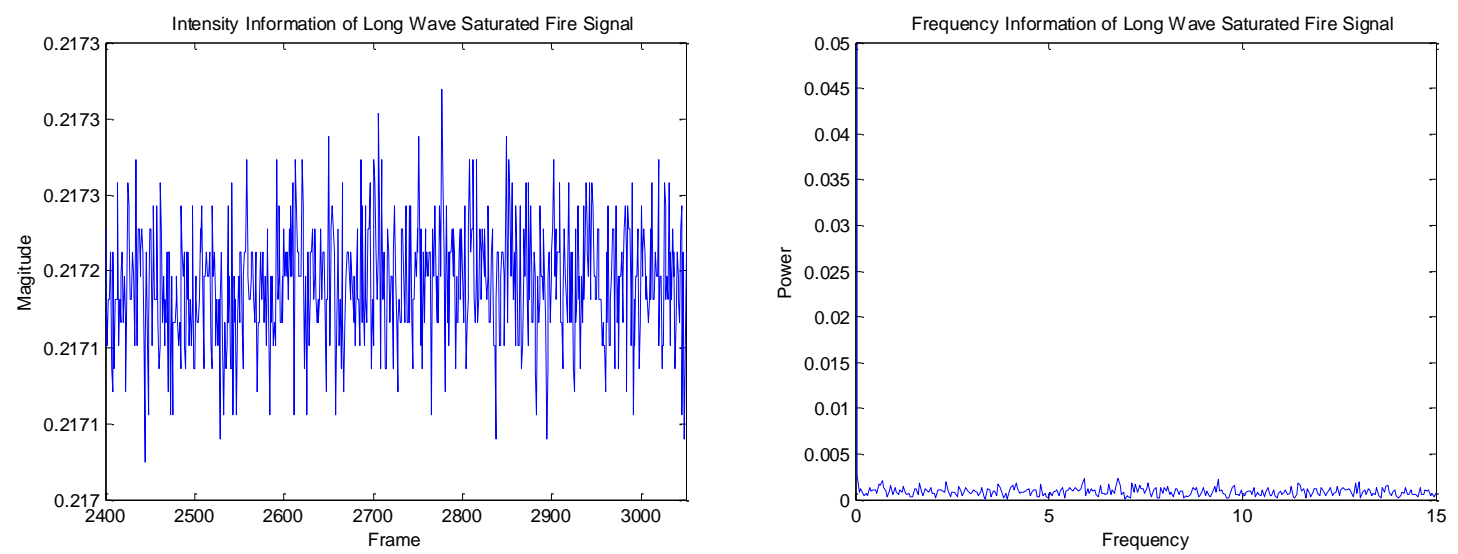

Figure 8 Temporal and Frequency Profile of a Saturated Fire Pixel

This means the pixel is a constant intensity value with a small amount of uniform white noise [18]. This implies that the sensor is in fact saturated and doesn't contain any useful frequency information. This does not negatively affect the algorithm because when the fire grows to this size a simple magnitude decision can detect the fire.

\subsection{Building fire signal}

The most important signal to look for is a building fire signal. This signal occurs when a pixel sees a fire but the fire is not strong enough to saturate the pixel. Frames 1 through 1085 of the oats 1 data display a good example of this type of signal. Figure 9 shows the time signal and frequency information of an unsaturated fire signal in both MWIR and LWIR. A DFT generates the frequency information. 

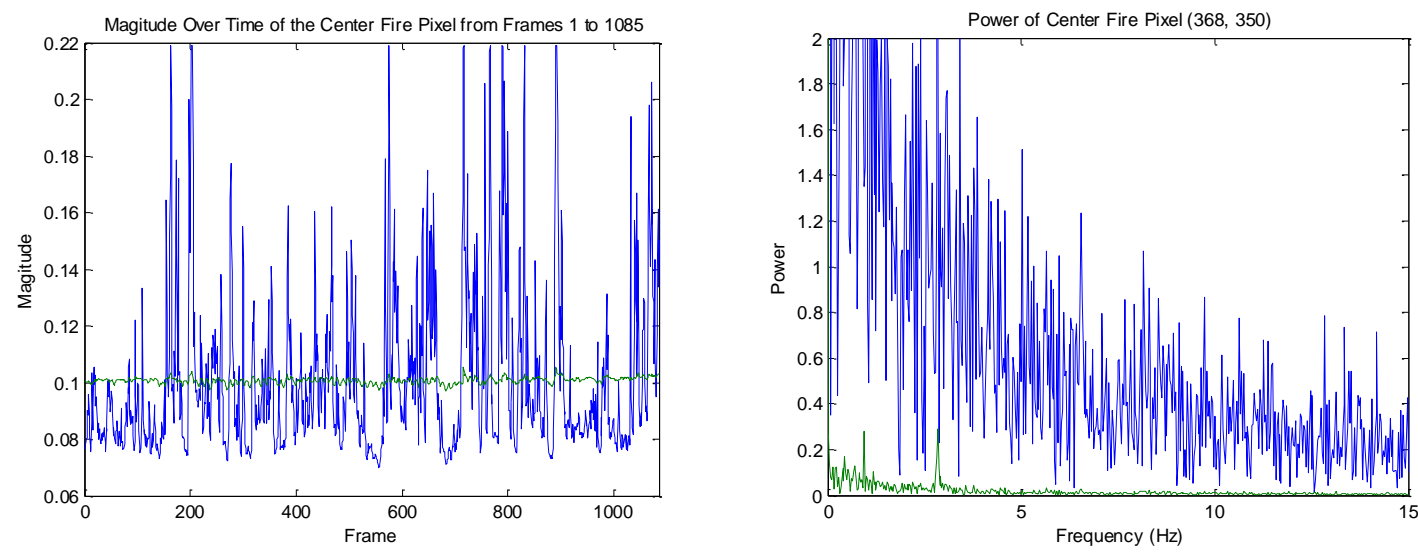

Figure 9 Temporal and Frequency Profile of Unsaturated Center Fire Pixel (MWIR is Green and LWIR is Blue)

In general the long wave fire signal is spread across the entire frequency spectrum with more power in the lower frequencies. The mid wave data generates a much smaller response. The mid wave data shows a much more constant value over the building fire frames. The mid wave data does show slightly more information in the lower frequencies. Next it is important to see how the signal changes further and further away from the center of the fire, pixel $(367,351)$. Figure 10 shows the frequency information looking further and further away from the center fire pixel measured by their city block [13] distance from the center fire pixel. The center fire pixel is also reshown on the same scale as the other plots for reference. 

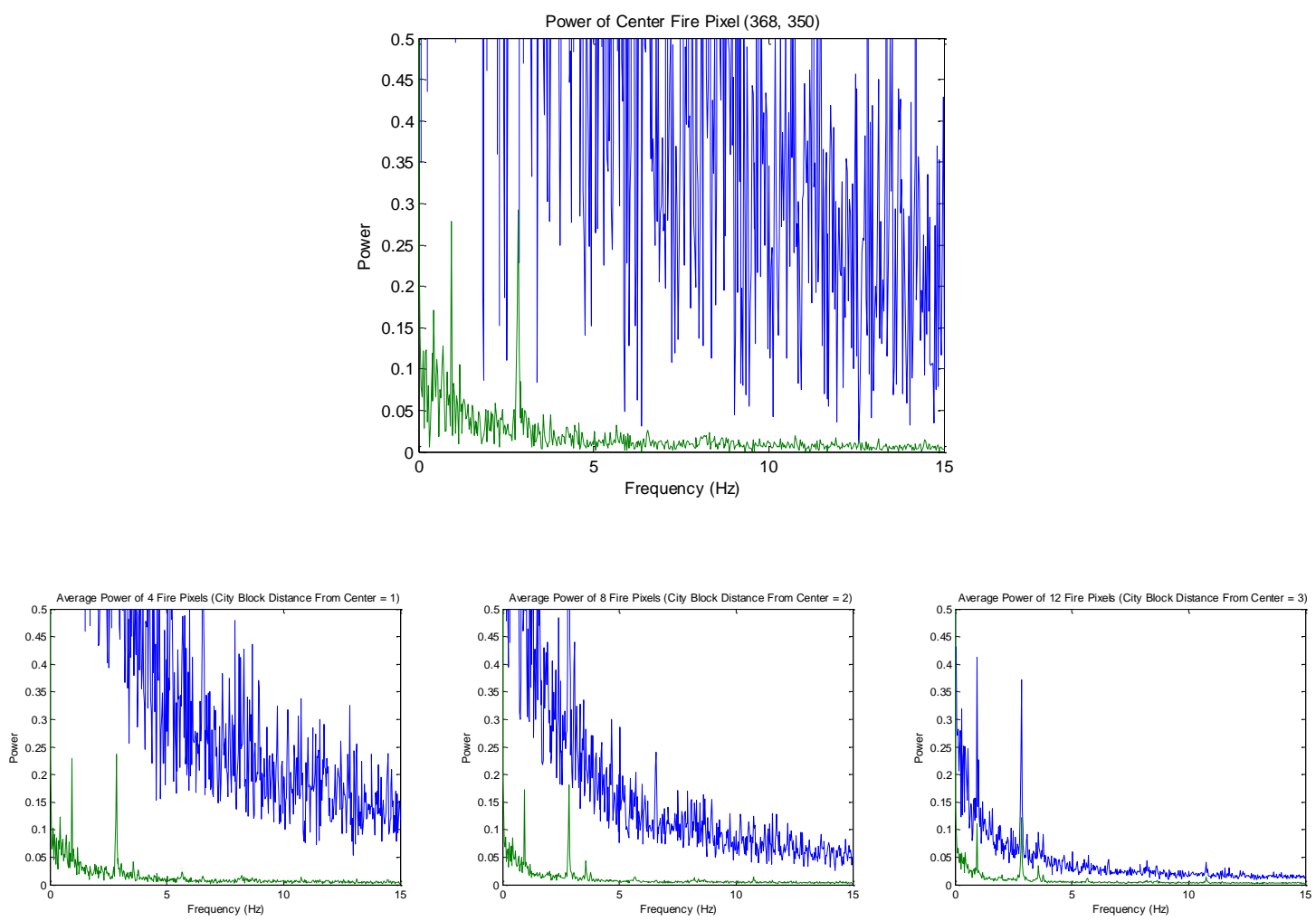

Figure 10 Frequency Profile of Pixels Moving Away From Center of Fire (MWIR is Green and LWIR is Blue)

In the long wave data the further away from the center of the fire the fire signal becomes smaller and smaller. The high frequencies disappear first and the lower frequencies remain present further away. Spikes at $.95 \mathrm{~Hz}$ and $2.84 \mathrm{~Hz}$ appear in both the long and mid wave data sets. This chapter identifies these signals later. The next three categories of non-fire pixels to look at are a uniform pattern, a textured pattern and a sharp edge pattern.

\subsection{Uniform Pattern}

The first non-fire pixel type to identify is one whose surrounding pixels are uniform. This pixel has the least amount of distortion due to the movement of the 
camera. Figure 11 shows 20 pixels near row 316 displaying the center of one of the building's hot roof. A DFT analyzes the frequency information for these pixels.

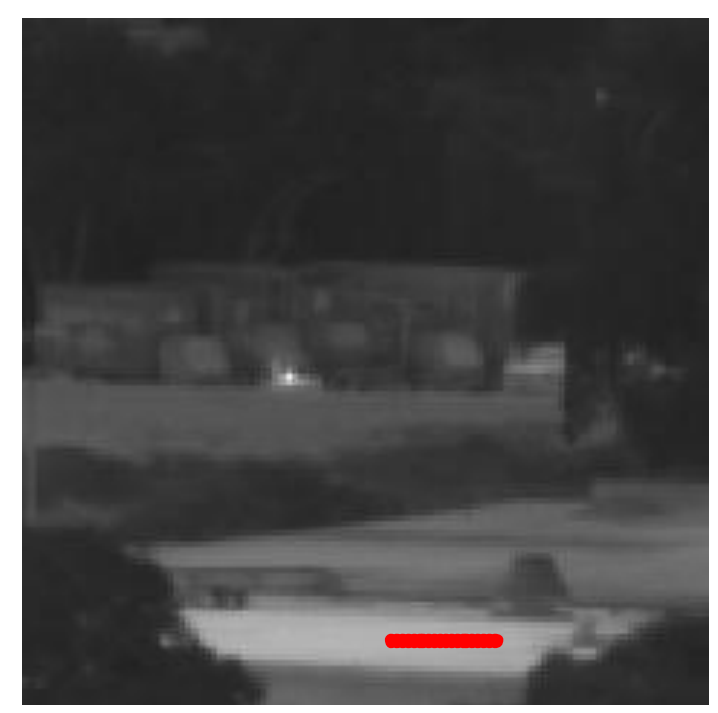

Figure 11 Uniform Pattern Pixels

Figure 12 shows the frequency results. As expected the frequency information displays a large DC component with a little bit of white noise. Two small frequencies do appear present in the spectrum. These frequencies are identified later in this section.

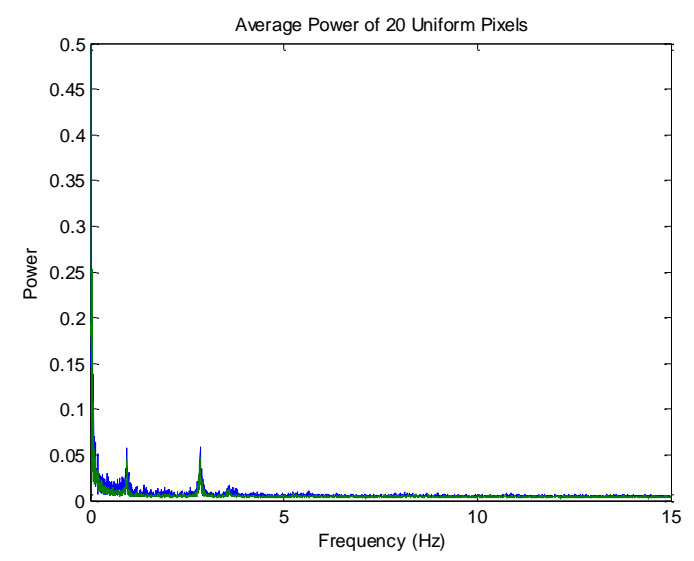

Figure 12 Frequency Profile of Uniform Location 
Next we look at a pixel near a sharp edge.

\subsection{Textured Pixel Signal}

Another type of signal to look for is a pixel looking at an area where the surrounding pixels have similar but not constant values. A good example of a textured pixel which generates a textured signal is the part of a frame looking at a tree. The pixels centered on pixel $(410,315)$ were analyzed as a textured surface. Figure 13 shows the selected region.

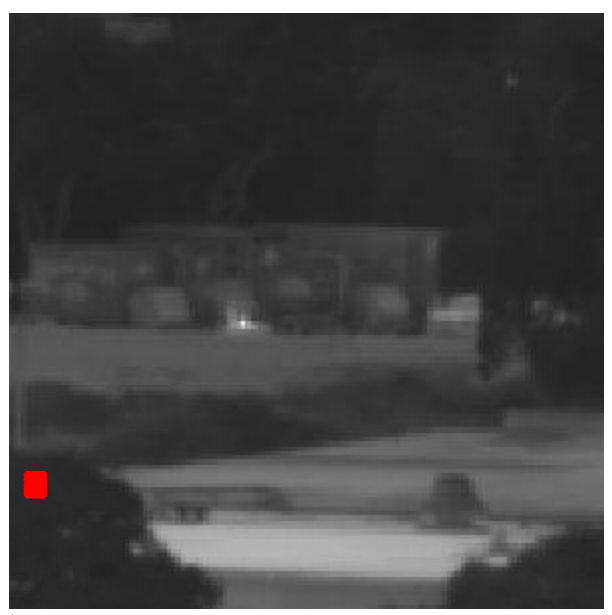

Figure 13 Location of Soft Edge Pixels

Figure 14 shows the frequency information of a textured surface. The plot on the right is set to the same scale as the other plots. 

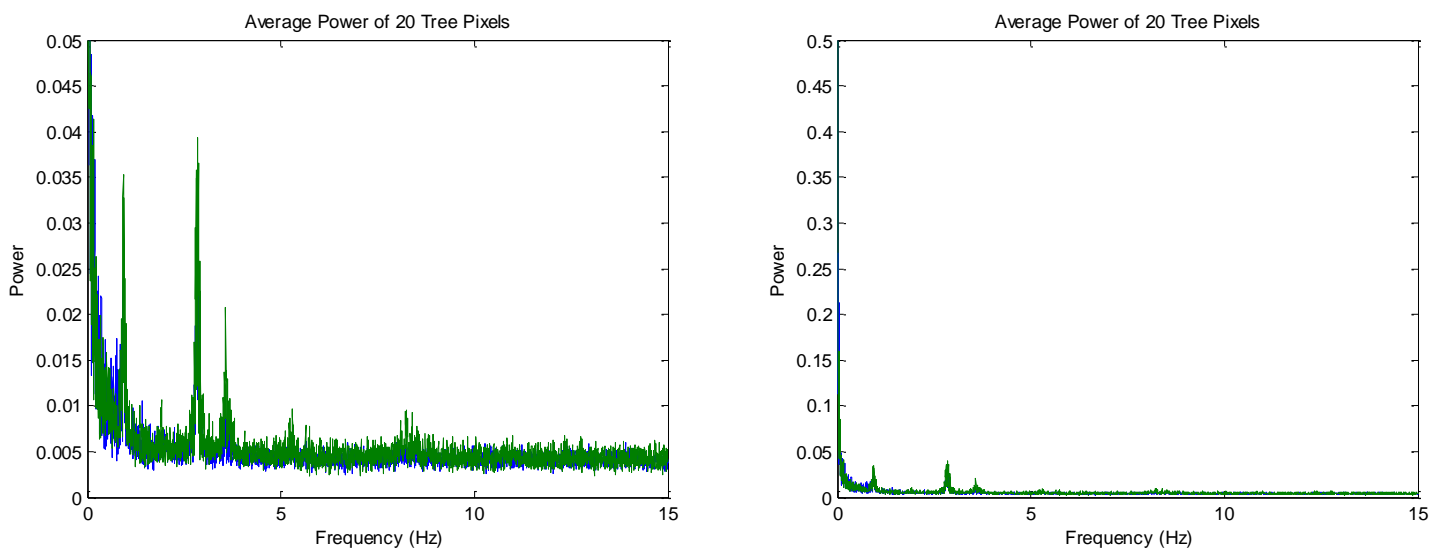

Figure 14 Frequency Profile of Textured Signals (MWIR is green and LWIR is Blue)

The textured edge's frequency appears very similar to that of a uniform signal. The movement of the tree leaves add very little if at all to the frequency spectrum. The same two frequency spikes appear and the next section explains their source.

\subsection{Strong Edge Signal}

The signal caused by a sharp edge proves to interfere the most with identifying the start of a fire. A pixel that alternatively looks at a hot pixel, like a roof top, and a cool pixel, like the side of a building in a shadow generates a sharp edge signal. As the camera moves in the wind a signal is created proportional to the resonate frequency of the tower that supports the camera. Any structure naturally resonates in multiple modes depending on their construction generating multiple frequencies [19]. Figure 15 shows 20 pixels that generate strong edge signals. 


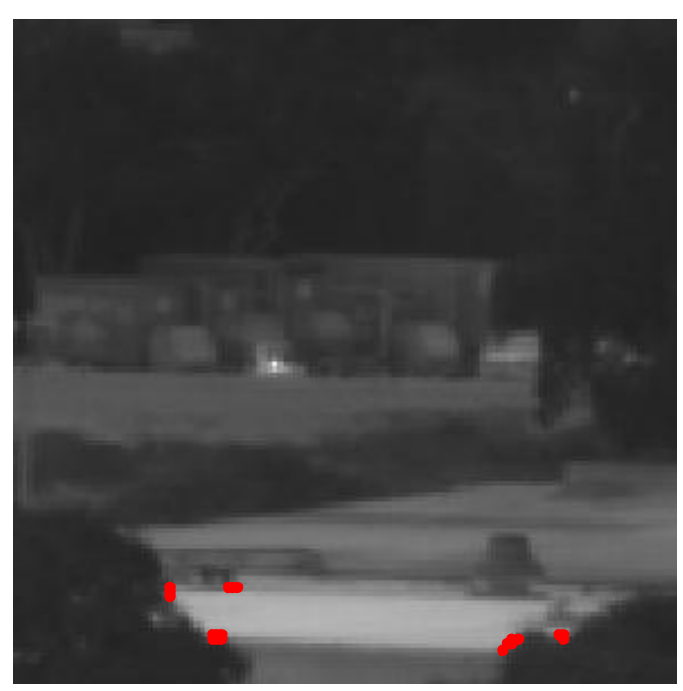

Figure 15 Location of Selected Strong Edge Pixels

An DFT was performed on these twenty pixels over 8000 frames to display their frequency information. Figure 16 shows the average frequency power of 20 of the strongest edges in the picture over the entire time period of the oats 1 data set. The blue line is generated from the long wave data and the green line is generated from the mid wave data. The spectrum generated shows nearly the same amount of power for both the long and mid wave data across the entire frequency spectrum. Both wavelengths show the same frequency spikes due to the tower resonance. The largest spikes are at $0.95 \mathrm{~Hz}$ and $2.84 \mathrm{~Hz}$. Smaller spikes are also found at $3.58 \mathrm{~Hz}, 5.30 \mathrm{~Hz}, 8.21 \mathrm{~Hz}$ and $10.75 \mathrm{~Hz}$. These frequencies match the earlier detected frequencies just at a much larger power. All of these frequencies need be avoided when looking for fire information. 

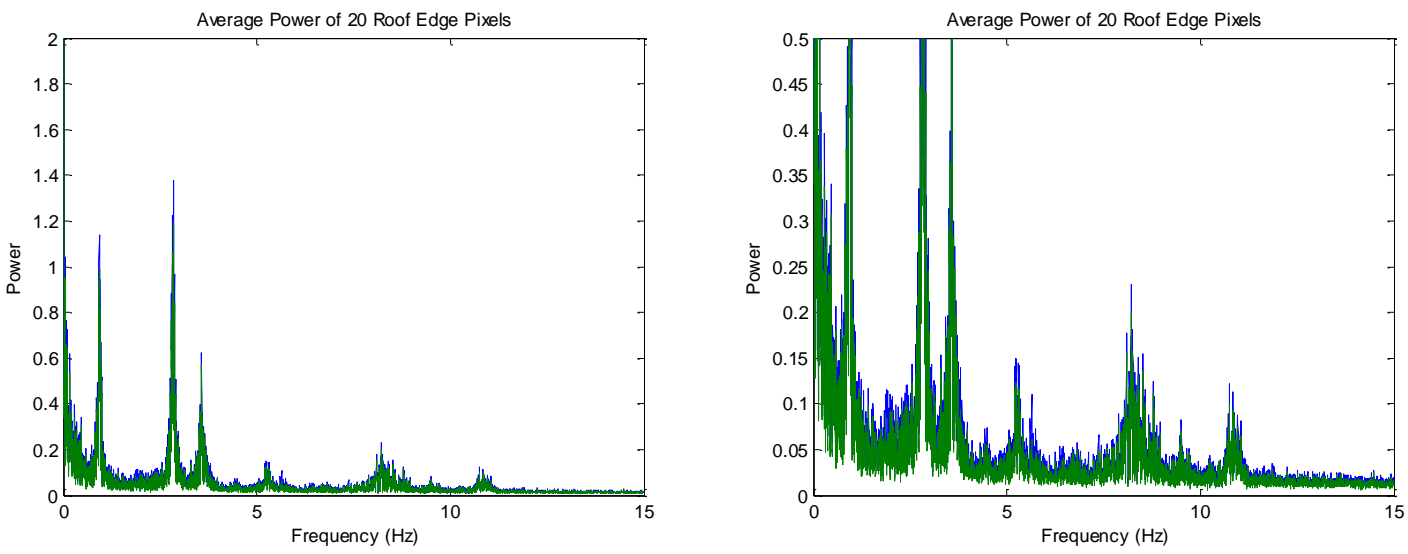

Figure 16 Frequency Profile of Strong Edge Signals (MWIR is green and LWIR is Blue)

\subsection{Moving Car Pixel}

The final type of pixel to analyze is the hardest to characterize in the frequency domain. All of the other pixel types had large consecutive run times and created steady state signals. Moving vehicles on the other hand were only visible by each pixel for a few frames at a time. They behave more like an impulse signal. During the time period between frame 1100 to 1300 the pixels around pixel 315, 400 witness two passing cars. Due to the short duration the frequency resolution is poor but there is some information that can still be gathered. Figure 18 shows the DFT of the data taken during these 200 frames. This plot is zoomed in to identify as much information about the signals as possible. 


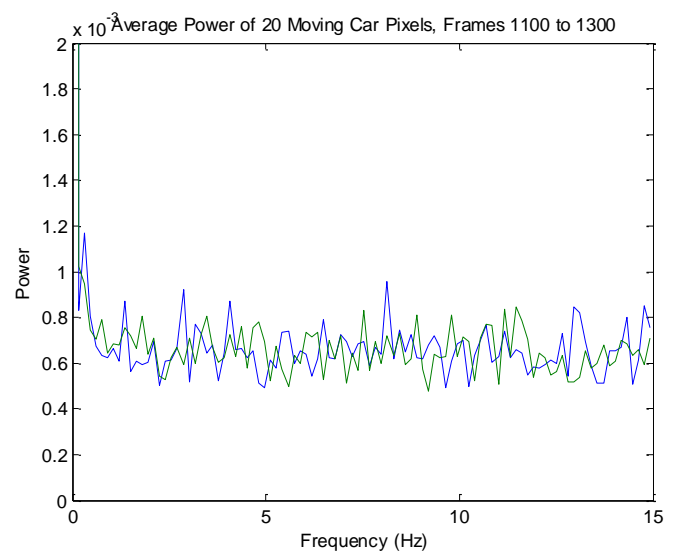

Figure 17 Frequency Profile of Moving Object Signals (MWIR is green and LWIR is Blue)

Most importantly the long and mid wave data have approximately the same amount of power throughout the frequency spectrum. This is similar to the edge results but very different from the fire pixel results. This makes sense knowing that a pixel observing a car passing in front of it is pretty much the same as a pixel observing an edge. The difference is an edge pixel creates periodic power while a moving object's has a flatter response. This fits the assumption that a moving object signal behaves similar to an impulse as the frequency response of an impulse has a constant value [20].

\subsection{Conclusion of Frequency Analysis}

A pixel with fire information contains frequency information in multiple sections in the frequency spectrum. The lower frequencies appear to contain more fire information in pixels further away from the center of the fire. Unfortunately signals generated by strong edges can overpower out any information about a small fire. A filter to best identify a fire needs to detect energy in a frequency area with minimal amount of edge frequencies and maximum amount of fire energy. Figure 18 shows two possible 
locations for filters that are ideal for detecting fires. A high pass filter will block almost the entire camera movement signal but will also block most of the fire's signal. A more ideal filter would be a band pass filter that captures the low frequency information between the peak at $0.95 \mathrm{~Hz}$ and the peak at $2.9 \mathrm{~Hz}$. This is a narrow band captures the most fire signal and a minimal amount of the tower movement signal. Unfortunately this lower frequency band contains more camera movement power than in the higher frequency area. Chapter 5 shows the design of filters for both of these frequency locations.
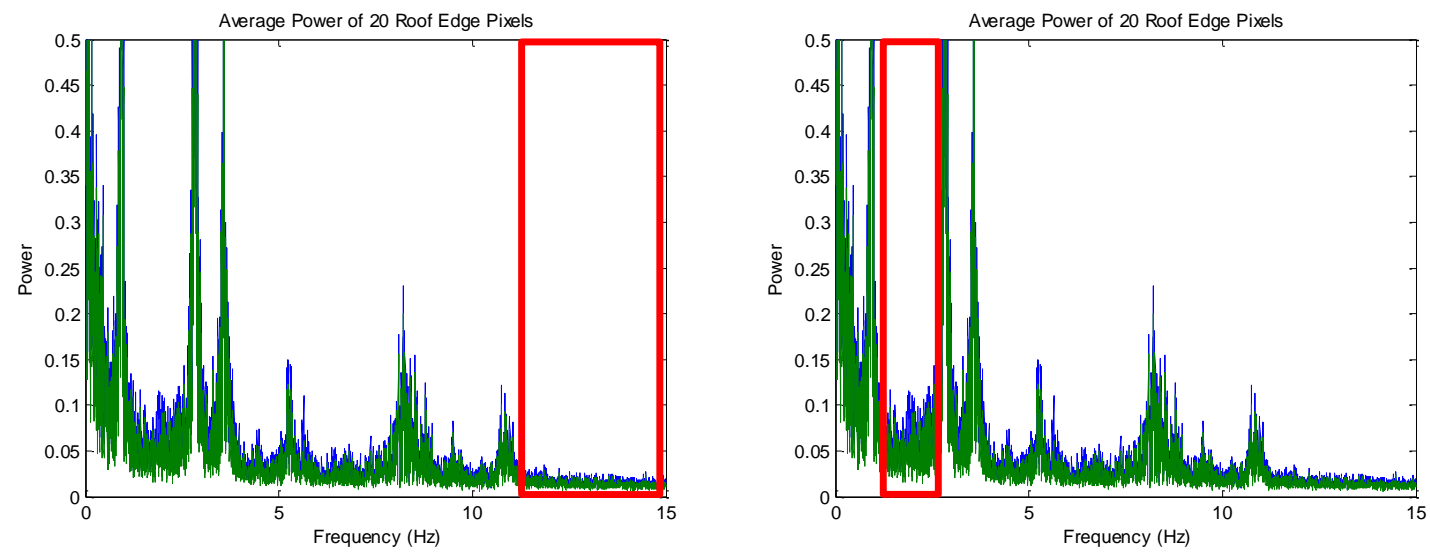

Figure 18 Location of Proposed Digital Filter Pass Bands 


\section{CHAPTER 5}

\section{Principals Used in Design of Fire Detection}

This chapter explains the theory used in each stage of the fire detection algorithm. The algorithm requires the design of the following stages: FIR high pass filter, IIR band pass filter, spatial blurring filter and connected component analysis.

\subsection{FIR High Pass Filter Design}

The design flow of creating a FIR filters is shown in Figure 19 [20]. The first set creates a low pass prototype filter. Multiplying the coefficients of the low pass prototype by a window function then increases the stop band performance of the filter. A transformation function then converts the low pass filter into the desired high pass filter.

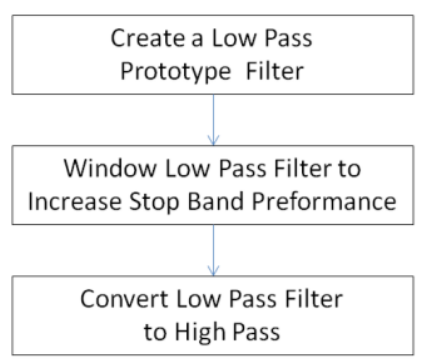

Figure 19 FIR High Pass Filter Design Procedure

\subsubsection{Creation of a Low Pass Prototype Filter}

The coefficients of any odd lengthened low pass filter of a given length can be found by using the following equations [20]:

$$
h_{\text {lowpass }}=2 * F_{c} * \operatorname{sinc}\left(2 * n * F_{c}\right) \text { where: } F_{c}=\text { cuttoff frequency }
$$




$$
\begin{gathered}
\text { where: } n=\left[\frac{-(M-1)}{2}, \frac{-(M-3)}{2}, \ldots, 0, \ldots \frac{M-3}{2}, \frac{M-1}{2}\right], \\
\text { given: } M=\text { filter length }
\end{gathered}
$$

Equation 1

\subsubsection{Window Low Pass Prototype}

Multiplying a window function to the low pass prototype helps improve the stop band performance of the filter. This is especially important for this filter as its job is to block the signals created by the edges. Any gain in stop band performance also causes an increase in the pass to stop band transition width. Table 3 shows the properties of 4 common window types where $\mathrm{N}$ is the filter length. Increasing the filter length improves the transition width but the pass band ripple and stop band attenuation can only improve by changing the window type.

Table 3 Window Function Characteristics [20]

\begin{tabular}{|l|l|l|l|}
\hline Window & $\begin{array}{l}\text { Peak Ripple } \\
\delta_{\mathrm{p}}=\delta_{\mathrm{s}}\end{array}$ & $\begin{array}{l}\text { Transition Width } \\
(\Delta \mathrm{f})\end{array}$ & $\begin{array}{l}\text { Stop Band Attenuation } \\
(\mathrm{dB})\end{array}$ \\
\hline Rectangle & 0.0897 & $0.92 / \mathrm{N}$ & -21 \\
\hline Hanning & 0.0063 & $3.21 / \mathrm{N}$ & -44 \\
\hline Hamming & 0.0022 & $3.47 / \mathrm{N}$ & -53 \\
\hline Blackman & 0.00017 & $5.71 / \mathrm{N}$ & -74 \\
\hline
\end{tabular}

\subsubsection{Convert Low Pass Prototype to a High Pass Filter}

The following formulas convert an odd length FIR low pass filter to a high pass filter [21]: 


$$
\begin{gathered}
h_{H P}\left(\frac{M+1}{2}\right)=-1 * h_{L P}\left(\frac{M+1}{2}\right)+1, \quad \text { given: } M=\text { filter length } \\
\boldsymbol{h}_{H P}\left(\boldsymbol{n} \neq \frac{\boldsymbol{M}+\mathbf{1}}{\mathbf{2}}\right)=-\mathbf{1} * \boldsymbol{h}_{L P}\left(\boldsymbol{n} \neq \frac{\boldsymbol{M}+\mathbf{1}}{\mathbf{2}}\right), \quad \text { given: } \boldsymbol{M}=\text { filter length }
\end{gathered}
$$

Equation 2

\subsection{IIR Filter Band Pass Design}

The most common way to design an infinite impulse response digital filter is to first design the desired analog filter and convert it to a digital filter [20]. Figure 20 shows a block diagram of the IIR filter creation process

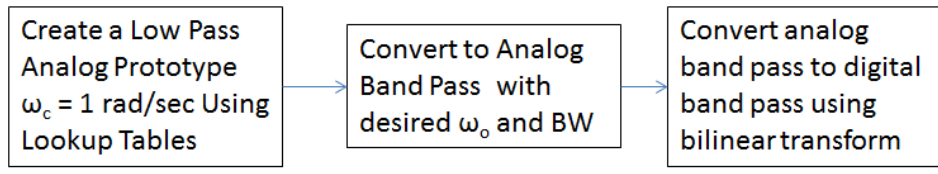

Figure 20 IIR Band Pass Design Procedure

\subsubsection{Create Analog Low Pass Prototype}

The creation of the analog low pass prototype is derived by using lookup tables for the desired filter type, Butterworth, Chebyshev, elliptical or others and the desired filter length [21]. Butterworth is characterized by a nominally flat pass and stop band while the Chebyshev contains variable ripple in the pass or stop band. Elliptical filter contain ripple in both the pass and stop band. In general the sharper the cutoff the greater the ripple in the pass and/or stop band. 


\subsubsection{Convert Analog Low Pass to Analog Band Pass}

Once the transfer function for the prototype filter is generated the following transformation will convert the prototype low pass filter transfer function into the desired analog band pass filter transfer function.

$$
H_{B P}(j \omega)=\left.H_{L P}(j \omega)\right|_{j \omega=Q\left(\frac{j \omega}{\omega_{o}}+\frac{\omega_{o}}{j \omega}\right)}
$$

$$
\text { where: } Q=\frac{\omega_{o}}{B W}, \omega_{o}=\sqrt{\omega_{1} * \omega_{2}}, B W=\omega_{2}-\omega_{1}
$$

Equation 3

\subsubsection{Bilinear Transform}

The next step converts the analog filter into a digital filter using a bilinear transform [20].

$$
\begin{gathered}
H_{\text {digital }}(z)=\left.H_{\text {analog }}(s)\right|_{s=C * \frac{z-1}{z+1}}, \quad \text { where } C=\tan \left[0.5\left(\Omega_{2}-\Omega_{1}\right)\right] \\
\Omega_{1}=\mathbf{2} * \boldsymbol{\pi} * \boldsymbol{F}_{\mathbf{1}}=\mathbf{2} * \boldsymbol{\pi} * \frac{\boldsymbol{f}_{\mathbf{1}}}{\text { Sample Rate }}
\end{gathered}
$$

Equation 4

The MATLAB commands "lp2bp" and "bilinear" preform the pervious transformations. These commands were used when designing the IIR filters for this thesis. 


\subsection{Gaussian Blurring Spatial Filter}

A Gaussian blurring spatial filter filters out any high noise in an image [22]. The filter itself is generated from a two dimensional Gaussian curve from the following function:

$$
\begin{aligned}
f(x, y)=\left(\frac{1}{2 \pi * \sigma_{x} * \sigma_{y}}\right) \\
\\
\quad * e^{-\left(\frac{\left(x-x_{o}\right)^{2}}{2 \sigma_{x}^{2}}+\frac{\left(y-y_{o}\right)^{2}}{2 \sigma_{y}^{2}}\right)}, \text { where }\left(x_{o}, y_{o}\right) \text { is the filter's center }
\end{aligned}
$$

Equation 5

There are two inputs in to this filter: the standard deviation and the filter size. For blurring purposes it makes sense for $\sigma_{\mathrm{x}}=\sigma_{\mathrm{y}}$. This blurs the image the same amount in the $\mathrm{x}$ and $\mathrm{y}$ direction. The filter size needs to be about 6 times the size of the selected standard deviation. Three standard deviations contain $98 \%$ of the total power of the function. Figure 20 shows the values of a 5 x 5 Gaussian filter with a standard deviation of 1 .

Table 4 Values for $5 \times 5$ Gaussian with $\sigma=1$

\begin{tabular}{|l|l|l|l|l|}
\hline 0.003 & 0.0133 & 0.0219 & 0.0133 & 0.003 \\
\hline 0.0133 & 0.0596 & 0.0983 & 0.0596 & 0.0133 \\
\hline 0.0219 & 0.0983 & 0.1621 & 0.0983 & 0.0219 \\
\hline 0.0133 & 0.0596 & 0.0983 & 0.0596 & 0.0133 \\
\hline 0.003 & 0.0133 & 0.0219 & 0.0133 & 0.003 \\
\hline
\end{tabular}

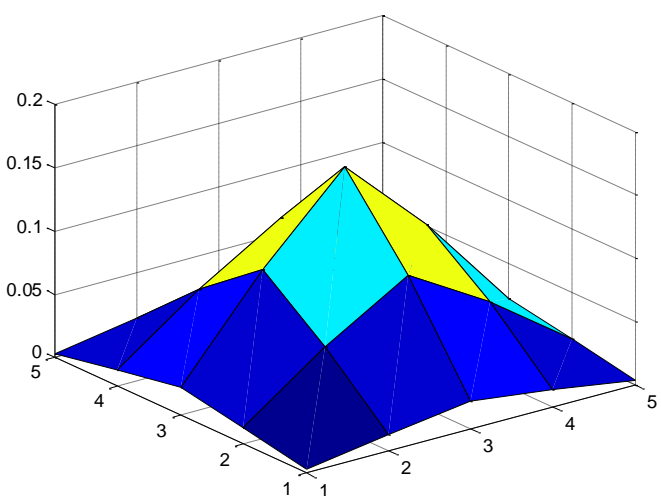

Figure 215 x 5 Gaussian with $\sigma=1$ 
To complete the blurring process the Gaussian filter is convoluted across the image. Blurring allows for improved results in the next step segmentation.

\subsection{Connected Component Analysis}

Connected component analysis converts a binary image into a labeled image where each object contains a different value [23]. This is a two-step process. The first set goes pixel by pixel from the top left looking for a true pixel values. Once detected the algorithm looks to the left, top left, top and top right for labeled pixels. If they exist the current pixel assumes that label number. If they don't the pixel assumes a new label number. Once completed a second pass identifies two touching labels. The algorithm then classifies each label as the same object. Below shows an example of the connected component analysis process. Figure 22 starts with the binary image.

\begin{tabular}{|l|l|l|l|l|l|l|l|l|l|}
\hline 0 & 0 & 0 & 0 & 0 & 0 & 0 & 0 & 0 & 0 \\
\hline 0 & 1 & 0 & 1 & 0 & 1 & 1 & 0 & 0 & 0 \\
\hline 0 & 1 & 1 & 1 & 0 & 1 & 1 & 0 & 1 & 0 \\
\hline 0 & 1 & 1 & 1 & 0 & 1 & 1 & 0 & 1 & 0 \\
\hline 0 & 0 & 0 & 1 & 0 & 0 & 0 & 0 & 1 & 0 \\
\hline 0 & 0 & 0 & 1 & 0 & 0 & 1 & 1 & 1 & 0 \\
\hline 0 & 1 & 0 & 0 & 0 & 0 & 0 & 0 & 0 & 0 \\
\hline 0 & 1 & 1 & 1 & 1 & 1 & 0 & 0 & 0 & 1 \\
\hline
\end{tabular}

Figure 22 Original Binary Image

Pixels are analyzed starting at the top left and going row by row. Figure 23 shows the results of pass one. 


\begin{tabular}{|l|l|l|l|l|l|l|l|l|l|}
\hline 0 & 0 & 0 & 0 & 0 & 0 & 0 & 0 & 0 & 0 \\
\hline 0 & 1 & 0 & 2 & 0 & 3 & 3 & 0 & 0 & 0 \\
\hline 0 & 1 & 1 & 1 & 0 & 3 & 3 & 0 & 4 & 0 \\
\hline 0 & 1 & 1 & 1 & 0 & 3 & 3 & 0 & 4 & 0 \\
\hline 0 & 0 & 0 & 1 & 0 & 0 & 0 & 0 & 4 & 0 \\
\hline 0 & 0 & 0 & 1 & 0 & 0 & 5 & 5 & 5 & 0 \\
\hline 0 & 6 & 0 & 0 & 0 & 0 & 0 & 0 & 0 & 0 \\
\hline 0 & 6 & 6 & 6 & 6 & 6 & 0 & 0 & 0 & 7 \\
\hline
\end{tabular}

\begin{tabular}{|l|l|l|l|l|l|l|l|l|l|}
\hline 0 & 0 & 0 & 0 & 0 & 0 & 0 & 0 & 0 & 0 \\
\hline 0 & 1 & 0 & 1 & 0 & 2 & 2 & 0 & 0 & 0 \\
\hline 0 & 1 & 1 & 1 & 0 & 2 & 2 & 0 & 3 & 0 \\
\hline 0 & 1 & 1 & 1 & 0 & 2 & 2 & 0 & 3 & 0 \\
\hline 0 & 0 & 0 & 1 & 0 & 0 & 0 & 0 & 3 & 0 \\
\hline 0 & 0 & 0 & 1 & 0 & 0 & 3 & 3 & 3 & 0 \\
\hline 0 & 4 & 0 & 0 & 0 & 0 & 0 & 0 & 0 & 0 \\
\hline 0 & 4 & 4 & 4 & 4 & 4 & 0 & 0 & 0 & 5 \\
\hline
\end{tabular}

Figure 23 Results of First Pass and Final Results of Connected Component Analysis

Pass two identifies 2 sets of labels sharing a common border. In this example labels 1 and 2 are the same as well as labels 4 and 5 . Figure 23 shows the final results with the labels renumbered for continuity. Throughout this thesis connected component analysis is performed using the MATLAB bw2label function. 


\section{CHAPTER 6}

\section{Design Process}

Two different filters need to be designed to capture the fire signal while rejecting the camera movement signal and the DC signal. The two different regions are show in Figure 18 in chapter 4 . The high pass filter captures some of the fire signal while avoiding almost the entire camera movement signal. The band pass filter captures much more of the fire signal but will also catch some the camera movement signal.

The are two different digital filter types to pick from when designing filters: a finite impulse response (FIR) filter and an infinite impulse response (IIR) filter. FIR filters have the advantage that they are always stable and can be designed with linear phase. IIR filters on the other hand can be designed with much sharper cutoffs with shorter filter lengths. The high pass filter is designed as a FIR filter and due to the small bandwidth the band pass filter is designed as an IIR filter.

\subsection{FIR High Pass Filter Implementation}

The FIR high pass filter requires three different parameters: - $6 \mathrm{~dB}$ cutoff frequency, filter length and window type. The most important parameter, and the one to look at first, is the windows type. This is the only parameter that controls the stop band attenuation. The filter needs to block the signals generated by the edges. The Hamming window block stop band signals with $-53 \mathrm{~dB}$ of attenuation. While the Blackman window preforms better in the stop band performance its transition width is almost twice that of the Hamming window and would lead to a significantly longer filter length. The 
longer filter length causes an increased number of computations and increased filter delay.

The next parameter for the high pass filter is the $-6 \mathrm{~dB}$ cutoff frequency. A $6 \mathrm{~dB}$ cutoff frequency of $13 \mathrm{~Hz}$ passes enough high pass power while still blocking the highest edge created frequency spike at $11 \mathrm{~Hz}$. This leads to a transition width of approximately $2 \mathrm{~Hz}$ analog frequency or $0.133 \mathrm{~Hz}$ in digital frequency. For a Hamming window with a transition width of $2 \mathrm{~Hz}$ requires a filter length of at least 25 . Figure 23 shows the response from high pass filters with lengths 21 through 31 for comparison.

Table 5 FIR High Pass Filter Legend

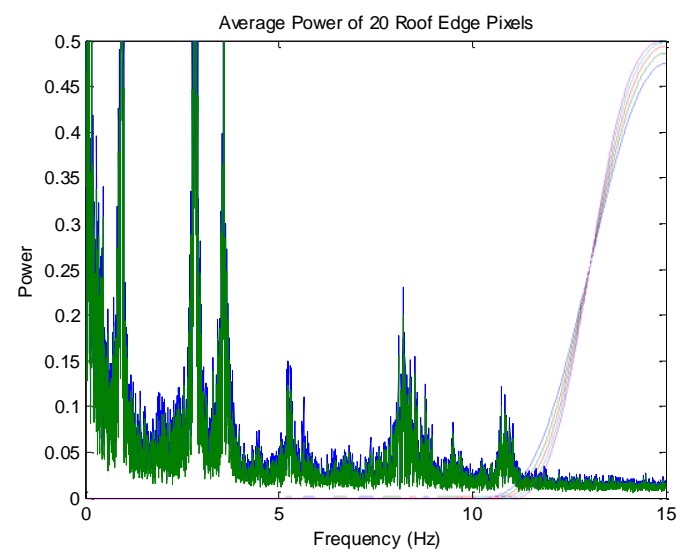

\begin{tabular}{|l|l|}
\hline Color & Filter Length \\
\hline Blue & 21 \\
\hline Green & 23 \\
\hline Red & 25 \\
\hline Cyan & 29 \\
\hline Purple & 31 \\
\hline
\end{tabular}

Figure 24 FIR High Pass Filter of Different

\section{Lengths}

Apon inspection of the different filter lengths a 29 length high pass filter is selected. The shorter transition width and flatter pass band gives improved performance. Figure 25 shows the final 29 length FIR high pass filter design. 

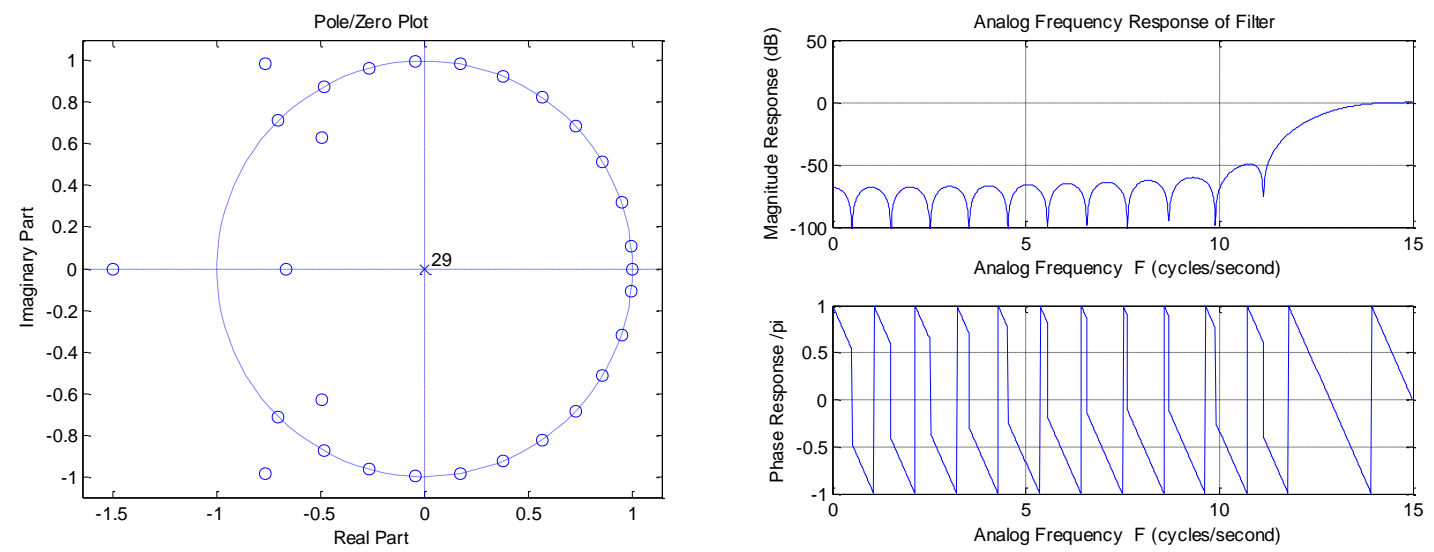

Figure 25 Final FIR High Pass Filter Design

The group delay of a symmetric FIR filter is found using Equation 6 .

Group Delay $=\frac{\text { FilterLength }-1}{2}=14$ frames $=.467$ seconds

Equation 6 
Equation 7 displays the final FIR high pass filter equation.

$$
\begin{gathered}
y[0]=\sum_{0}^{28} B_{k} * x[-k] \text { where }: \\
B_{k}=
\end{gathered}
$$

\begin{tabular}{|c|c|c|c|c|c|}
\hline k & $B_{k}$ value & k & $B_{k}$ value & $k$ & $B_{k}$ value \\
\hline 1 & -0.00083 & 12 & -0.09205 & 22 & -0.00556 \\
\hline 2 & 0.002179 & 13 & 0.113571 & 24 & 0.008271 \\
\hline 3 & -0.00423 & 14 & -0.12817 & 25 & -0.00855 \\
\hline 4 & 0.006682 & 15 & 0.133333 & 26 & 0.006682 \\
\hline 5 & -0.00855 & 16 & -0.12817 & 27 & -0.00423 \\
\hline 6 & 0.008271 & 17 & 0.113571 & 28 & 0.002179 \\
\hline 7 & -0.00407 & 18 & -0.09205 & 29 & -0.00083 \\
\hline 8 & -0.00556 & 19 & 0.067096 & & \\
\hline 9 & 0.021271 & 20 & -0.04245 & & \\
\hline 10 & -0.04245 & 21 & 0.021271 & & \\
\hline 11 & 0.067096 & 23 & -0.00407 & & \\
\hline
\end{tabular}

Equation 7

\subsection{IIR Band Pass filter Implementation}

In creating the band pass filter, the two large frequency spikes at $0.9 \mathrm{~Hz}$ and 2.9

$\mathrm{Hz}$ need to be almost completely blocked. In order to block these frequencies a band pass filter need to have $3 \mathrm{~dB}$ cutoff frequencies at $1.4 \mathrm{~Hz}$ and $2.2 \mathrm{~Hz}$ or a center frequency of $1.8 \mathrm{~Hz}$ and a bandwidth of $0.8 \mathrm{~Hz}$. This bandwidth is impractical to achieve with a FIR band pass filter. From Table 1 a FIR band pass filter would require a filter length of at least 249 using a Hamming window. 


$$
\Delta f=\frac{B W}{2}(\text { minimum })=\frac{0.8 / 2}{\text { sample rate }}=0.0133, \quad \Delta f=\frac{3.3}{N}, \quad N=247.5
$$

Equation 8

An IIR filter can achieve a similar result with a much shorter filter length. The key performance parameters to look for are the stop band attenuation at the two edge spike frequencies, $0.9 \mathrm{~Hz}$ and $2.9 \mathrm{~Hz}$, as well as the total group delay of the filter. The higher order filter will achieve a narrower pass band to stop band transition but will also increase the total filter group delay.

Before the order of the filter can be determined an analog prototype must be selected. For this filter a Butterworth prototype was selected. A Butterworth filter has a maximally flat response in both the pass and stop band [21]. This is ideal for our filter because all the frequencies in the pass band contribute to the fire's power equally. The maximally flat stop band ensures none of the frequency spikes pass. Other filter types have stop band ripple and if one of these ripples line up with one of the edge frequency spikes the filter will also pass the edge signal power to the output. An additional benefit of using a Butterworth prototype is it achieves a mostly linear phase response in the pass band region which leads to a more constant group delay.

After the analog prototype filter selection the next step sets the filter order. In general the higher the order leads to a quicker pass to stop band transition but also increases the filter length and group delay. Figure 26 shows the filter response of a $1^{\text {st }}$ to $5^{\text {th }}$ order band pass filter overlaid on top of the DFT of the edge pixels. 
Table 6 Performance of IIR Band Pass Filters

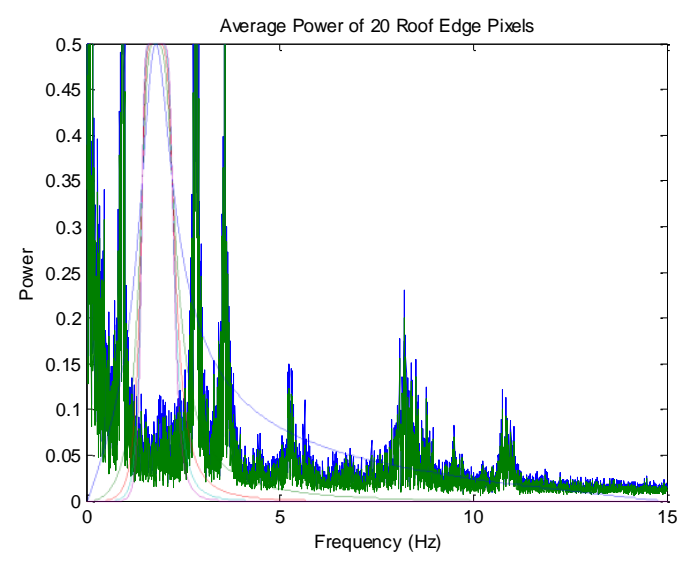

\begin{tabular}{|l|l|l|l|l|l|}
\hline Order & Color & $\begin{array}{l}\text { Low Spike } \\
\text { Gain }\end{array}$ & $\begin{array}{l}\text { Low Spike } \\
\text { Magnitude }\end{array}$ & $\begin{array}{l}\text { High Spike } \\
\text { Gain }\end{array}$ & $\begin{array}{l}\text { High Spike } \\
\text { Magnitude }\end{array}$ \\
\hline 1 & Blue & 0.3004 & 0.342756 & 0.3997 & 0.550787 \\
\hline 2 & Green & 0.09872 & 0.11264 & 0.1868 & 0.25741 \\
\hline 3 & Red & 0.03123 & 0.035633 & 0.0826 & 0.113823 \\
\hline 4 & Cyan & 0.00984 & 0.011227 & 0.03611 & 0.04976 \\
\hline 5 & Purple & 0.0031 & 0.003537 & 0.0157 & 0.021635 \\
\hline
\end{tabular}

Figure 26 IIR Band Pass Filters of Different Orders

The most important region the filter needs to block is the large edge frequency spikes at $0.95 \mathrm{~Hz}$ and $2.84 \mathrm{~Hz}$. The total power passed at these frequencies need to be significantly less than the power pass at the center of the pass band where the filter gain is 1 . Using the long wave data the total power passes at the center frequency of $1.7 \mathrm{~Hz}$ is 0.04487. A fourth order or larger filter blocks enough of the edge frequency spikes.

Figure 27 shows the group delay for a $1^{\text {st }}$ through $5^{\text {th }}$ order band pass filter. 
Table 7 Band Pass Filter Group Delay

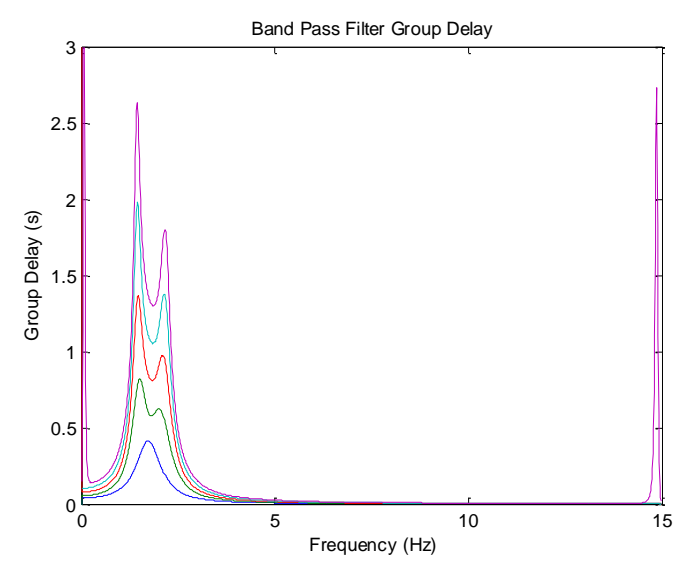

\begin{tabular}{|l|l|l|l|}
\hline Order & Color & $\begin{array}{l}\text { Max Pass Band } \\
\text { Group Delay (sec) }\end{array}$ & $\begin{array}{l}\text { Ave. Pass Band } \\
\text { Group Delay (sec) }\end{array}$ \\
\hline 1 & Blue & 0.4165 & 0.2535 \\
\hline 2 & Green & 0.8202 & 0.5459 \\
\hline 3 & Red & 1.3687 & 0.8407 \\
\hline 4 & Cyan & 1.9795 & 1.1334 \\
\hline 5 & Purple & 2.6348 & 1.4241 \\
\hline
\end{tabular}

Figure 27 Group Delay of IIR Band Pass Filters

Because of the properties of IIR filters the group delay is not going to be constant, instead each frequency passes through the filter in a different amount of time. As shown in Table 7 even changing the order length by one dramatically lengthens the delay of the filter.

Between a fourth and fifth order filter the average pass band delay increases by a quarter second while the maximum delay increases by over half a second. A $4^{\text {th }}$ order filter provides the best balance between performance and delay. Figure 28 shows the final design of the band pass filter. 

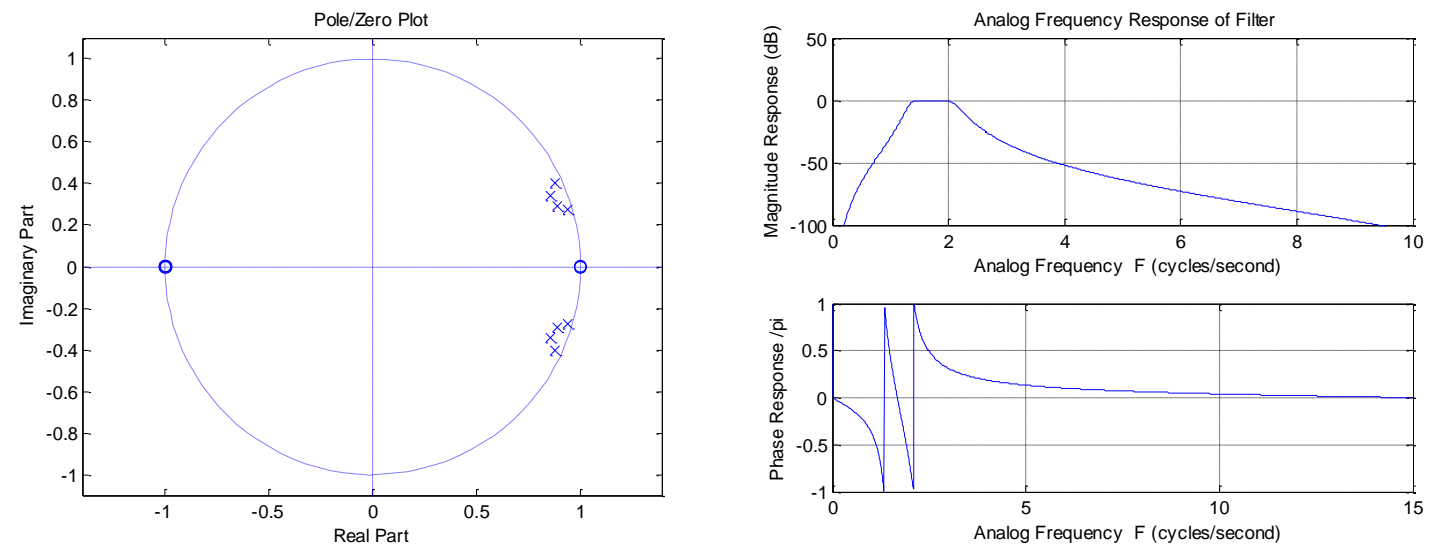

Figure 28 Final Design of IIR Band Pass Filter

Equation 9 displays the final IIR band pass filter equation.

$$
y[0]=\sum_{0}^{9} B_{k} * x[-k]-\sum_{1}^{9} A_{k} * y[-k], \quad \text { where }:
$$

$$
\begin{aligned}
& A_{k} \\
& =[1.0000,-7.1113,22.558,-41.6524,48.9387,-37.4610,18.2471,-5.1737,0.6544] \\
& B_{k} \\
& =[0.0352,-0.0000,-0.1407,-0.0000,0.2110,-0.0000,-0.1407,-0.0000,0.0352]
\end{aligned}
$$

Equation 9

\subsection{Location Specific Gain}

Unfortunately neither the high pass nor the band pass filter completely removes

all of the edge signals in the image. Some pixels look at edge rich locations while others 
look at more uniform areas. Because some of the edge power is passed the pixels in an edge filled area will always pass more power than a uniform area. Figure 29 show the frequency power for both long wave and mid wave bands over the first 250 frames ( 8 seconds) of the oats1 data generated using a DFT.
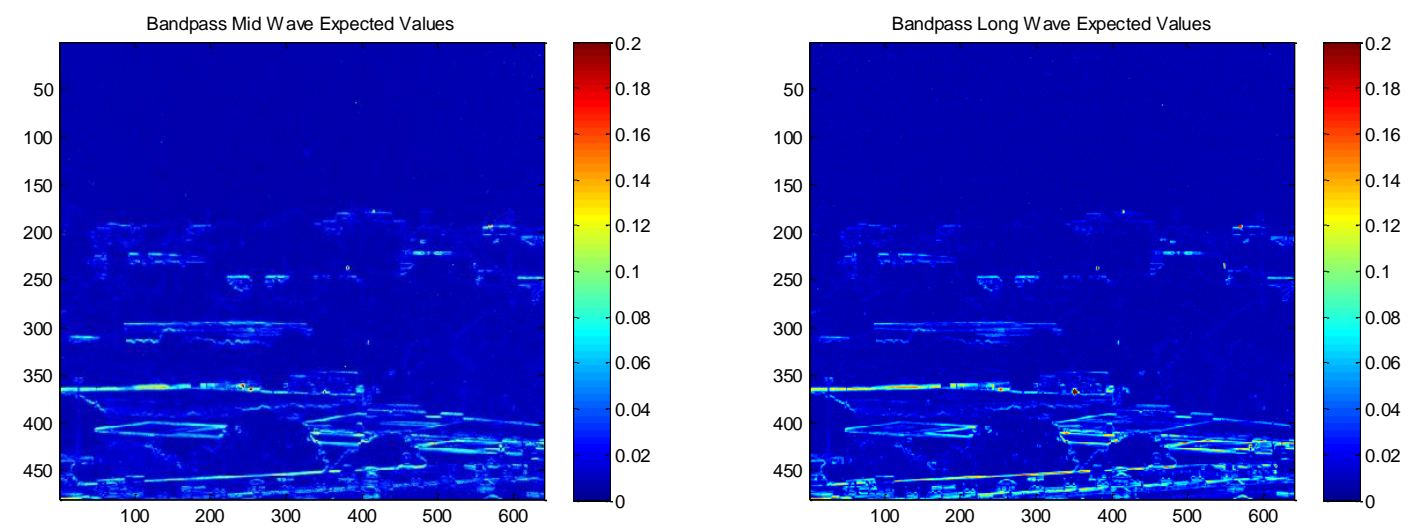

Figure 29 Expected Values for frequencies in the Band Pass Region for Both Long and Mid Wave

Unfortunately during these frames the fire is already present noted by the red dot near pixel $(351,367)$. We can simulate how the image would look by replacing the pixels contaminated by the fire with values of the surrounding areas. To ensure no false data is generated the altered values are set slightly higher than the surrounding areas. 

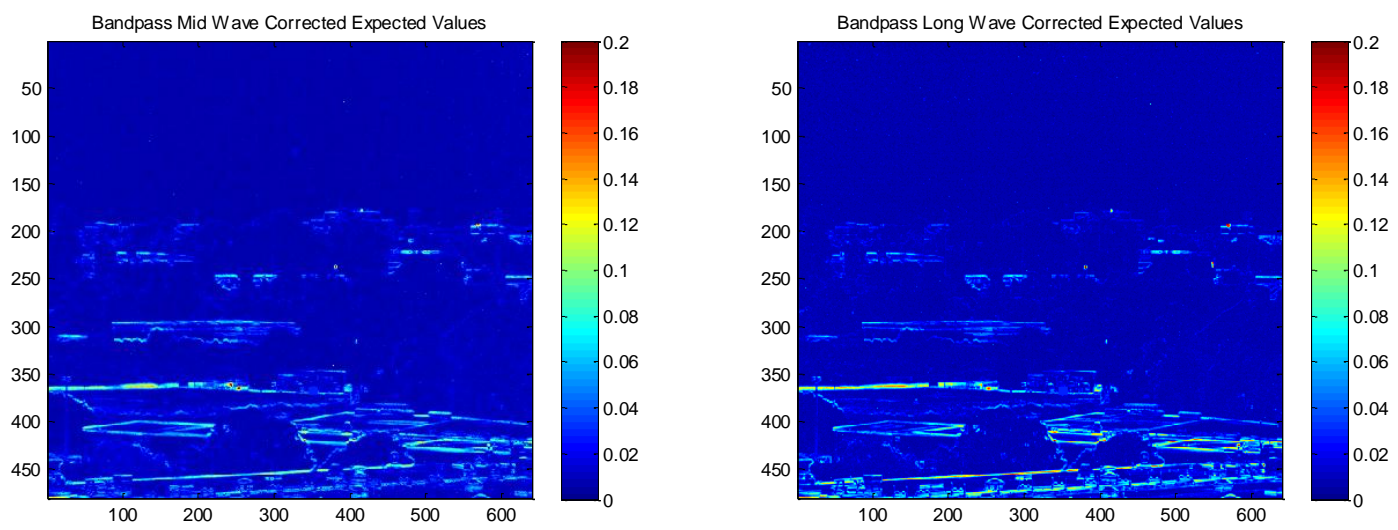

Figure 30 Corrected Values for Both Long and Mid Wave

These images were then normalized so the power of an average pixel is 1 . When the filter output is divided by these values the average power output of the filter needs to be approximately the same for each pixel in the frame allowing one threshold value for all pixels in the frame. This increases the sensitivity of the uniform areas, the red colored areas, while decreasing the sensitivity in the edge filled areas, the dark blue areas. The same procedure is performed for the high pass frequency range. 

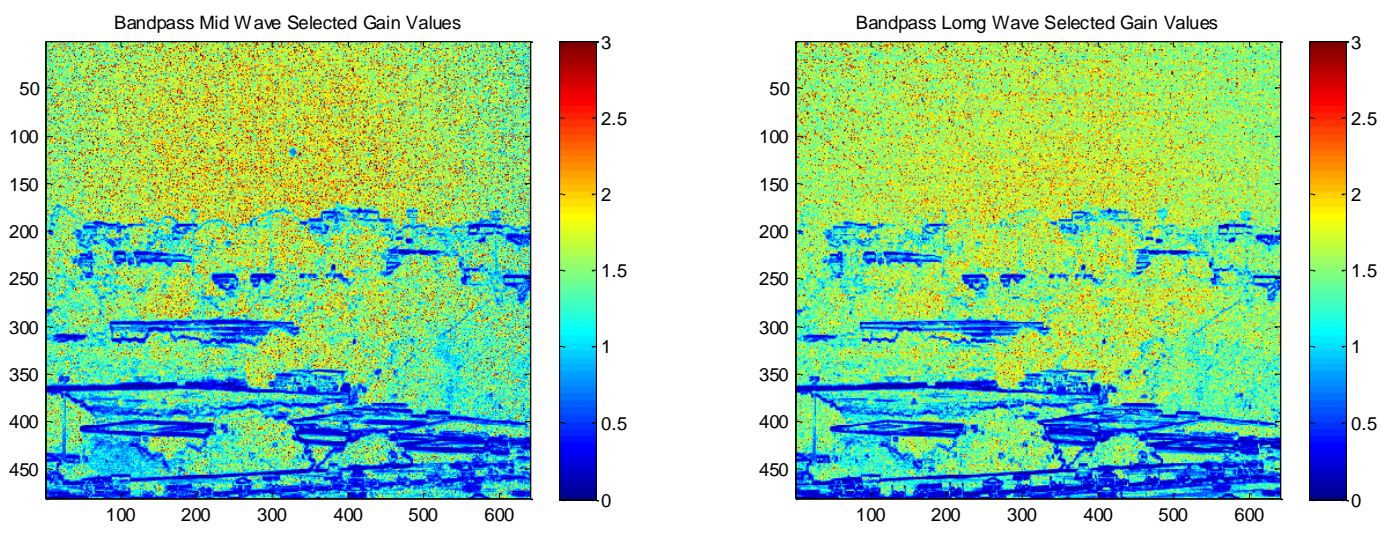

Figure 31 Pixel Specific Gain for both Long and Mid Wave band pass filter

\subsection{Spatial Filter and Connected Component Analysis}

The results of the location specific gain produce non-smooth images. This proves especially true for pixels displaying moving objects. For the connected component analysis to work correctly all pixels belonging to one object need to be connected. A spatial filter smooths out the images removing high frequency noise and which forms connected objects. Both the long wave and mid wave images are spatially filtered with a Gaussian filter with a standard deviation of 1 .

Once blurred a threshold is then set to only keep pixel values above a certain level as objects creating a binary image. A connective component analysis then breaks the binary image is separate each distinct object. Features are then generated for each object. Figure 32 shows the process from temporal filtered image to blurred image and finally to binary image taken from the frame 4000 of the charcoal 1 data set using the high pass filter. 

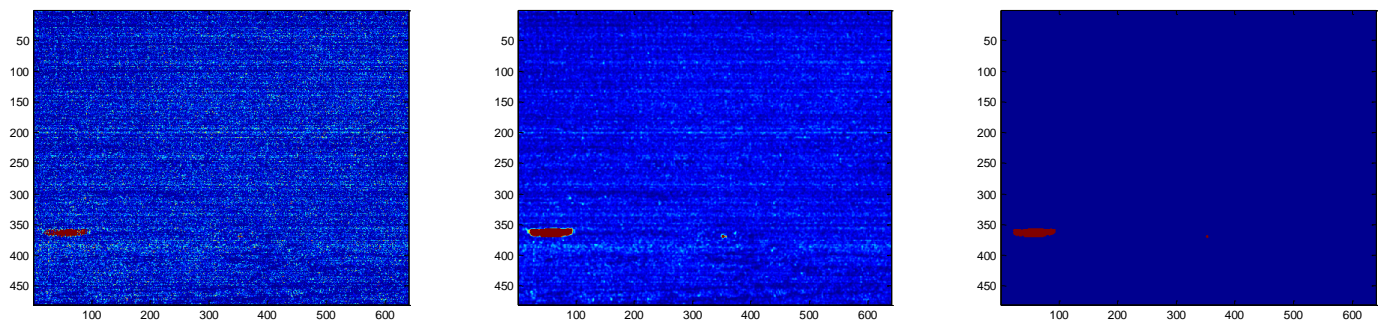

Figure 32 High Pass Original Image, Blurred Image and Binary Image

\subsection{Fire Detection by Features}

Once the objects are defined through the threshold process each one needs to be classified as either a fire or non-fire object. Non-fire objects can include moving objects, edges or just noise. Figure 33 shows the decision flow for categorizing each object.

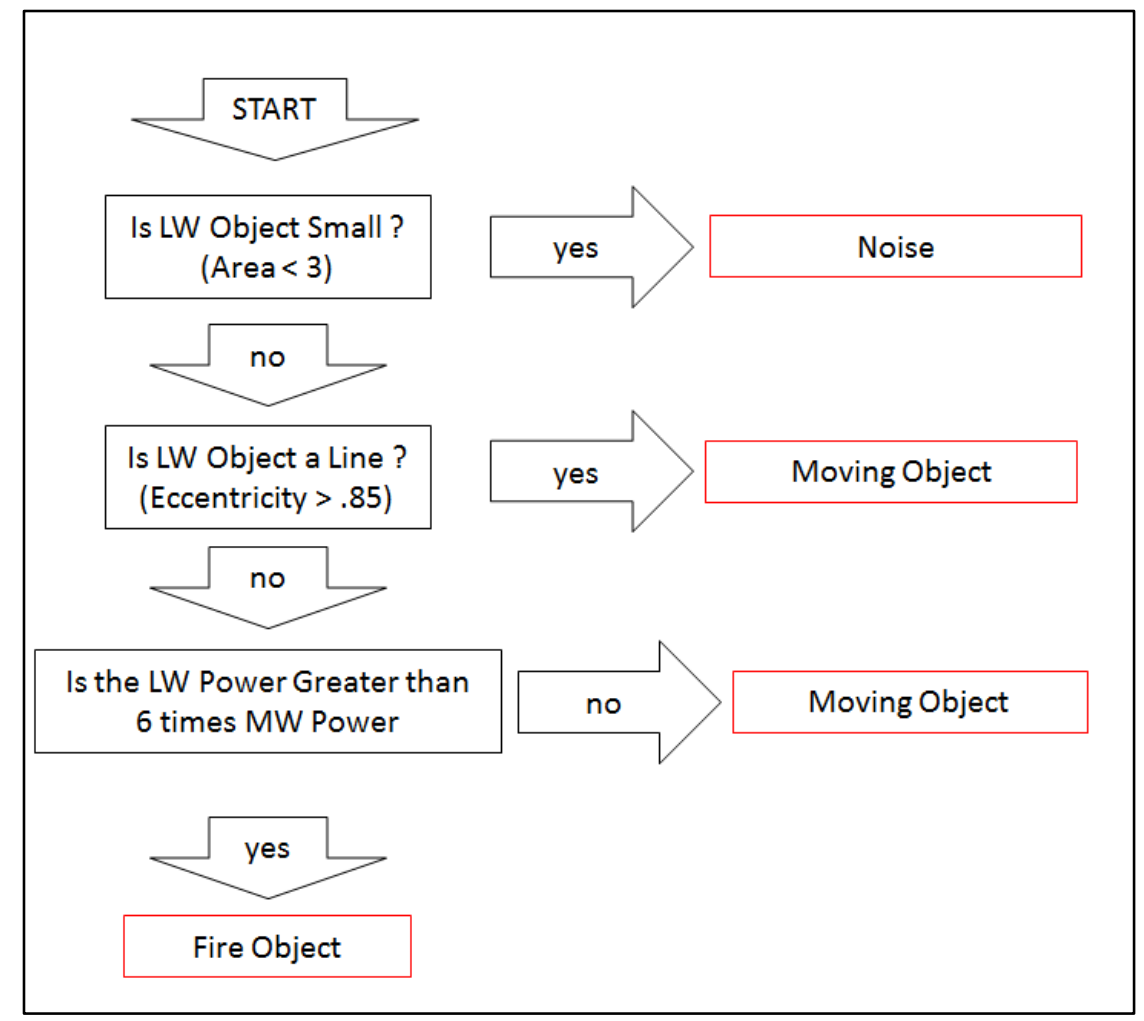

Figure 33 Decision Tree for Detected Objects 
The first type of object to remove is small area noise. It is impossible to determine enough information about objects with small number of pixels. Because of this the algorithm assumes any object less than three pixels in size contain noise and can be safely ignored. If these pixels contain fact fire signals within a few frames the fire will grow large enough to pass this stage in the decision tree.

The next object to attempt to remove is a moving object. There are two different ways this algorithm removes moving objects. The first method makes a decision off the object's shape. Because a moving object travels across several pixels the object appears as a blur across the path that it travels. Figure 34 shows a plane moving across the frame after being filtered by both the FIR high pass filter and the IIR band pass filter.
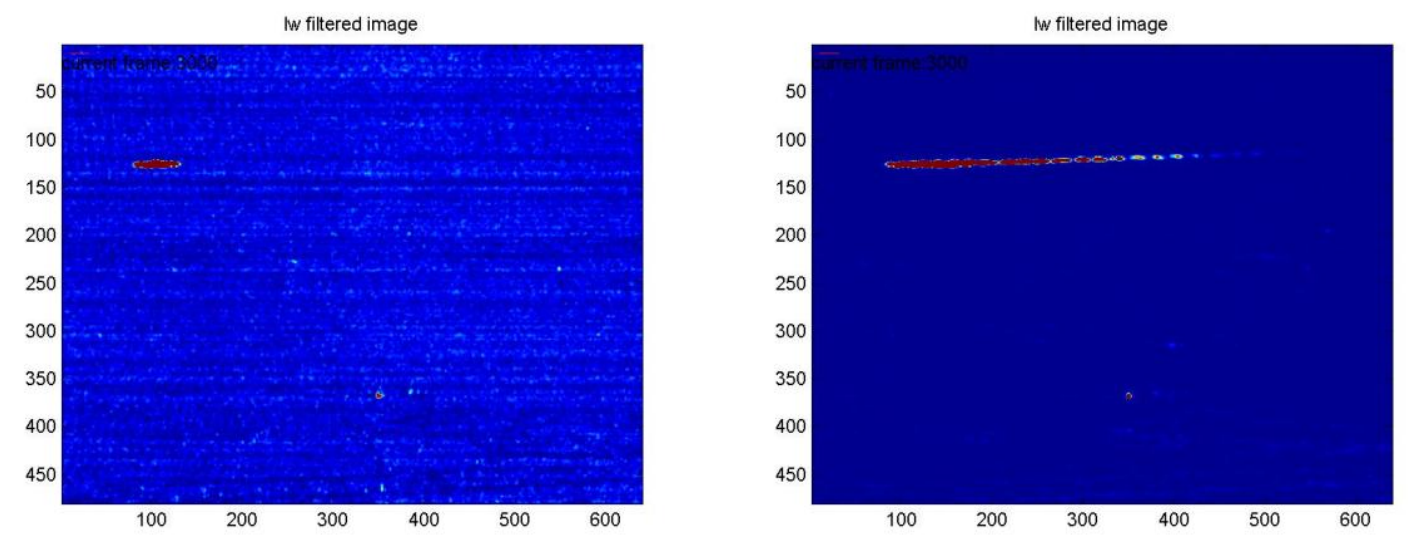

Figure 34 Moving Object (High Pass on Right Band Pass on Left)

Moving objects create a much larger blur in lower frequencies, the band pass image, than higher frequencies, the high pass image.

Each moving object typically has a large major axis and a small minor axis. Eccentricity ratio of the foci points of the major and minor axis [24]. A circle's 
eccentricity is zero and for a line it is one. For this algorithm if an objects eccentricity is greater than 0.85 the object is considered moving and rejected. This removes most of the moving objects but not all. Remaining areas containing edge signals also tend to have higher eccentricity and get rejected at this stage.

To remove the rest of the moving objects the mw wave data is used. As shown in chapter 4 earlier the pixels looking at the fire contain more long wave frequency information than the mid wave data. This means more long wave pixels will be above the threshold than mid wave pixels; the long wave object will contain more power than the mid wave object. Figure 35 show the power of the fire at frame 118 of the oats 1 data set for both the long and mid wave images generated by the band pass filter.
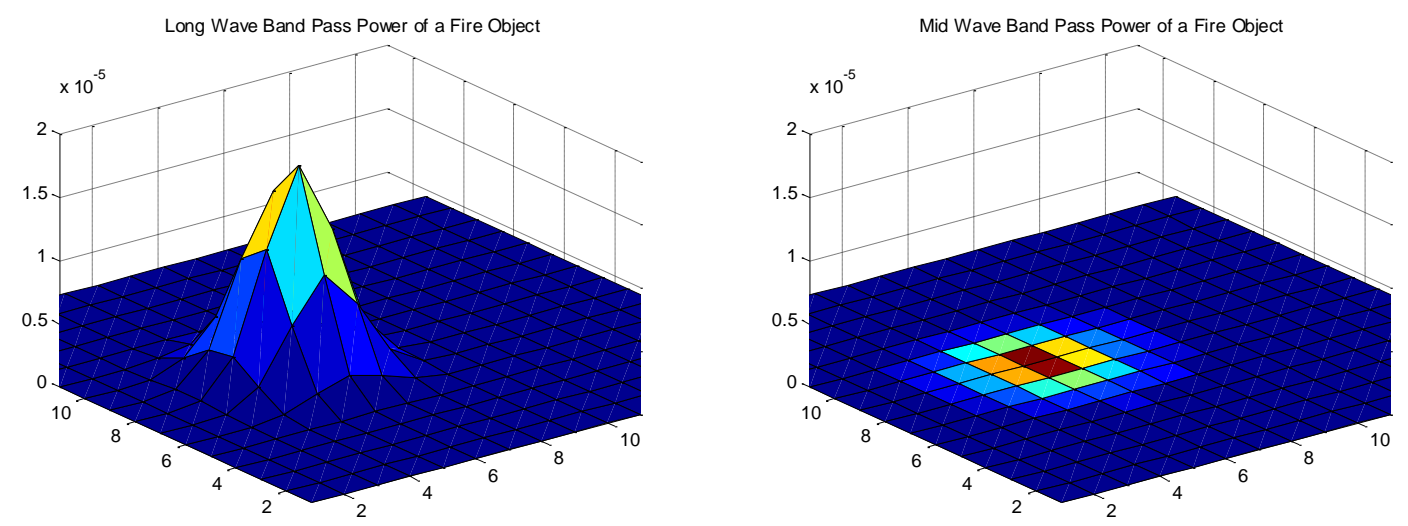

Figure 35 Long Wave vs. Mid Wave Power of a Small Fire

Moving objects on the other hand produce close to the same amount of power in both the long and mid wave image. Figure 36 shows the power from a moving object in the same frame as above. 

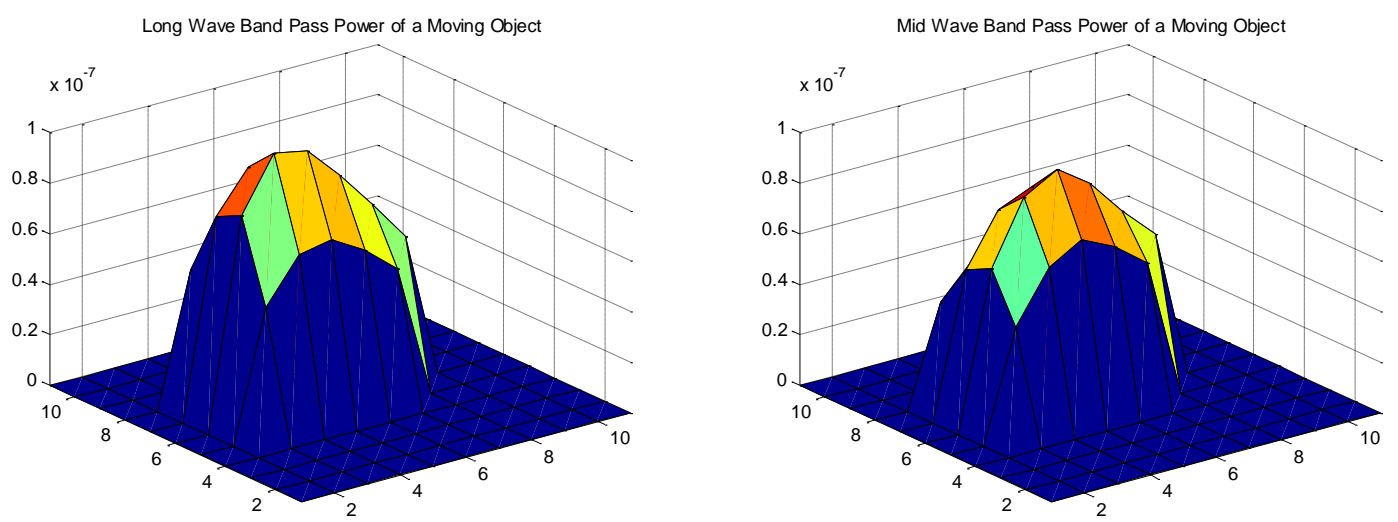

Figure 36 Long Wave Vs. Mid Wave Power of a Moving Object

This algorithm assumes that any object which contains more than 6 times the power in the long wave image over the mid wave image is a fire object. Any object failing this test contains signals generated by a moving object.

\subsection{Time Averaging Filtering}

The pervious step removed almost all of the false positives but some still remain. These false positives usually disappear after one or a few frames. The last step remove the noise generate by these false positives. A running average removes most signals which only get passes for one or a few frames. The final stage filters the results using a 120 frame (4 seconds) running average filter.

An actual implementation should require an even longer averaging filter. A longer averaging filter eliminates more of the false positives and the delay would make little or no difference in fire fighter response time. The short four second running averaging filter better tests how well the detection method works and to identify what circumstances could generate a false positive. 


\section{CHAPTER 7}

Results

Complete results for all the data sets are display in Appendix A. This section focuses on the results from the oats 1 and charcoal 1 data set. The charcoal 1 data set is the first set taken on the day of the experiment and is the only set that changes from a completely cool site to a heated site. Every other data set has been preheated from the data taken before. The oats1 data set is analyzed because this is the set that all the previous algorithms were tested against. As one of the earlier runs the metal barbeque didn't have enough time to get too hot. This data set also includes a greater variety of fire conditions. It includes moments of minimal fire activity as well as times of a more intense raging fire.

To trigger a positive fire reading the algorithm needs to record at least 10 pixels of fire activity. The only time this really applies in the charcoal data. Every other data set contains an active fire at frame 1 . The group delay of the filter causes to only delay in fire detection. The total number of fire pixels contains important information though; the more pixels detected means the further away the fire could be detected and how much of the fire could be block and still be detected.

The results graphs show the total number of fire pixels for the high pass filter, the band pass filter as well as a war LWIR result. The raw LWIR result display the total number of LWIR pixels 1.1 times the hottest non-fire pixel through the data det. As the day progressed the tops of the buildings heated up so the threshold had to change for each 
data set. In practice this is an unreliable way to detect a fire but does work as a baseline for showing the effectiveness of the algorithm.

As the testing day progressed the LWIR/MWIR camera started to overheat and could not maintain its 30 frames per second frame rate. The frame rate dropped down to as low as 18 frames per second in some cases [11]. The digital filters cease to preform correctly once the sampling rate changes. Because of this only data up to the palm data set contains useful data. As the sampling rate changes the edge frequencies caused by the camera movement appear to change. Figure 37 shows the same 20 edge pixels from both the oats1, early in the day, and applewood1 data, late in the day.
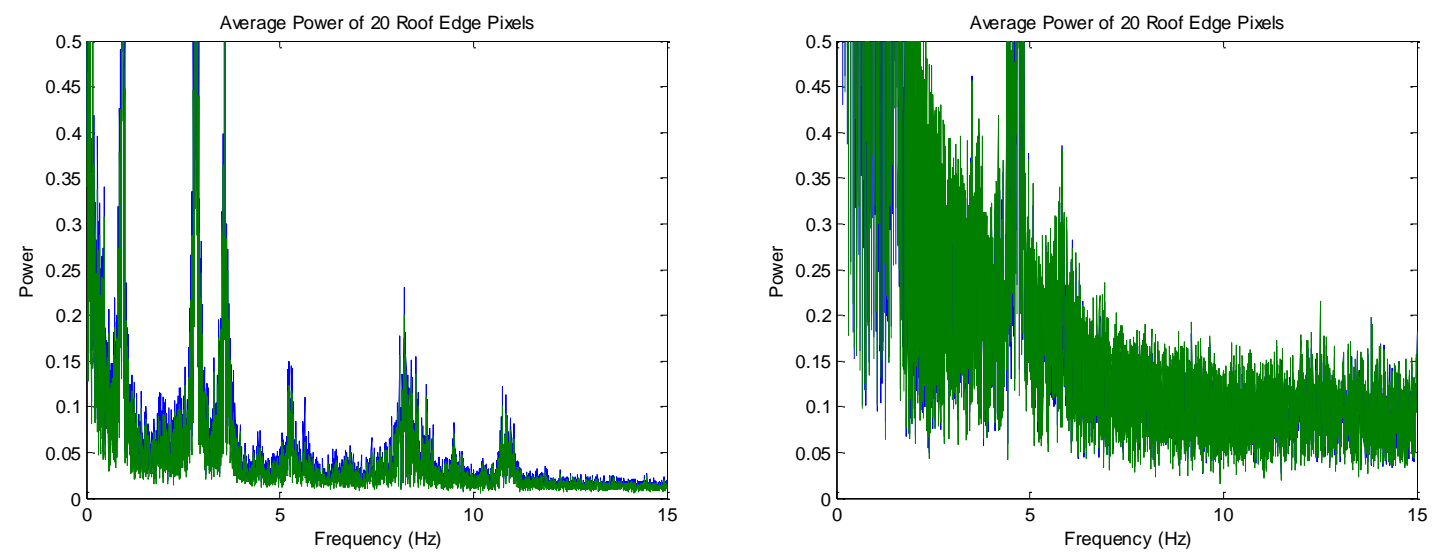

Figure 37 Strong Edge Frequencies for Stable Sample Rate (Left) Vs. Failed Sample Rate (Right)

Due to the changing sampling rate the primary edge frequency appears to shift from 3.58 $\mathrm{Hz}$ to $4.7 \mathrm{~Hz}$ and considerable noise corrupts the rest of the temporal frequency spectrum. These conditions make it impossible to use frequency filtering to positively identify fire pixels. Figure 38 shows the results from a failed frame rate in the applewood 1 data set. The edge signals overpower any other signals in the image. 


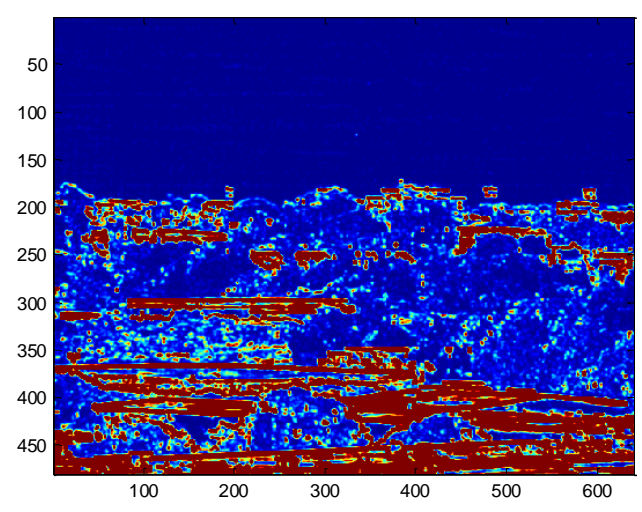

Figure 38 Results due to Failed Frame Rate

\subsection{Charcoal1 Results}

The charcoal data set by far had the smallest and most difficult fire to detect.
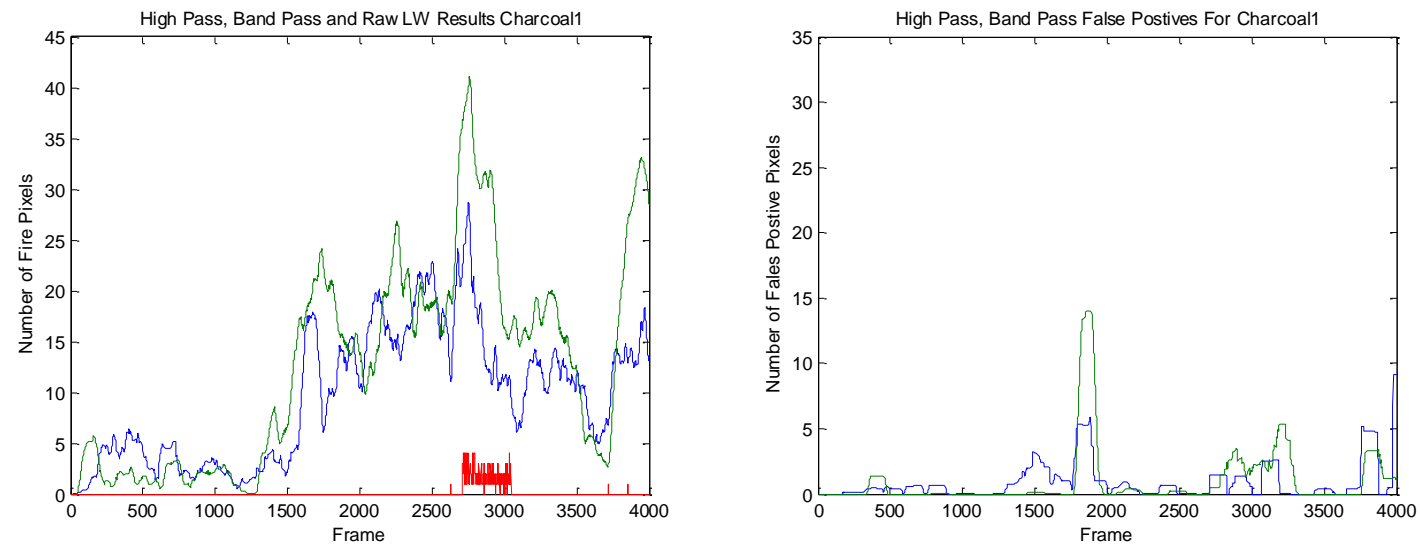

Figure 39 shows the results from both the high pass and low pass filter. 

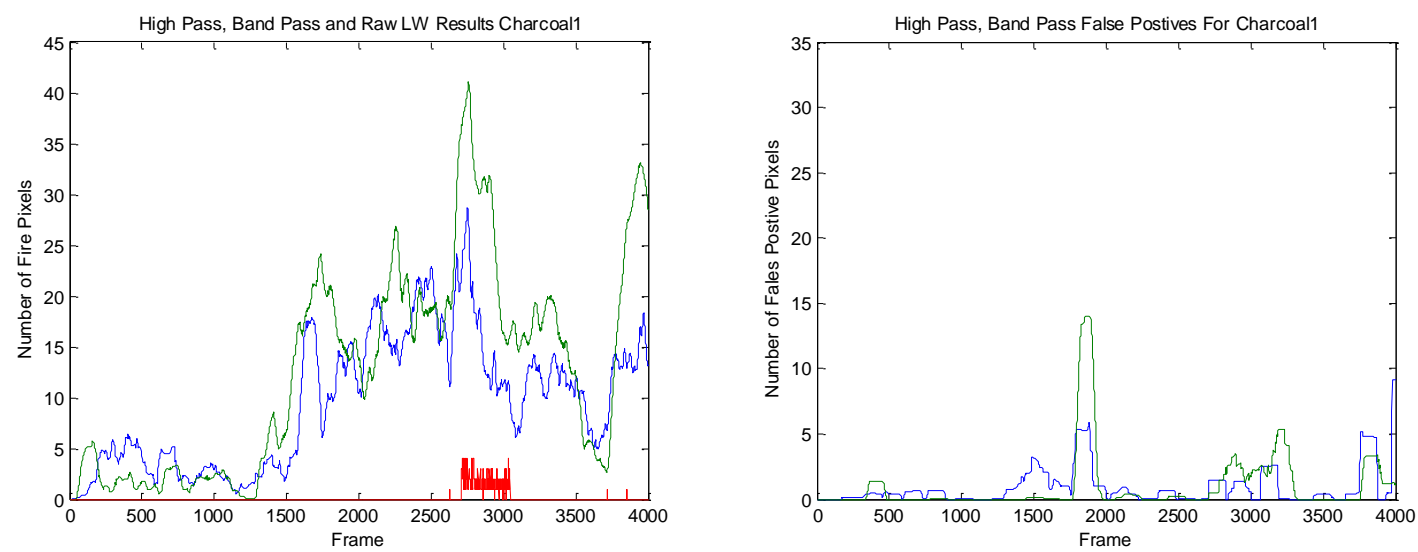

Figure 39 Charcoal1 Results Band Pass is Green and High Pass is Blue

The high pass filter detects a fire at frame 1598 and the band pass first detects the fire at frame 1526. Even with the increased group delay of the filter the band pass filter detects the fire almost two and a half seconds quicker than the high pass filter. Both filters outperform the raw long wave intensity threshold which doesn't detect the fire until 2706. The band pass filter detects the fire almost 40 seconds before the raw long wave intensity threshold does. Once the fire becomes large enough to register on both filters, the filter results are similar.

\subsection{Oats1 Results}

The oats 1 data set displays a much larger variety of fire conditions but has a visible fire starting at frame 1. Figure 40 shows the results from both filters and a raw long wave intensity threshold. 

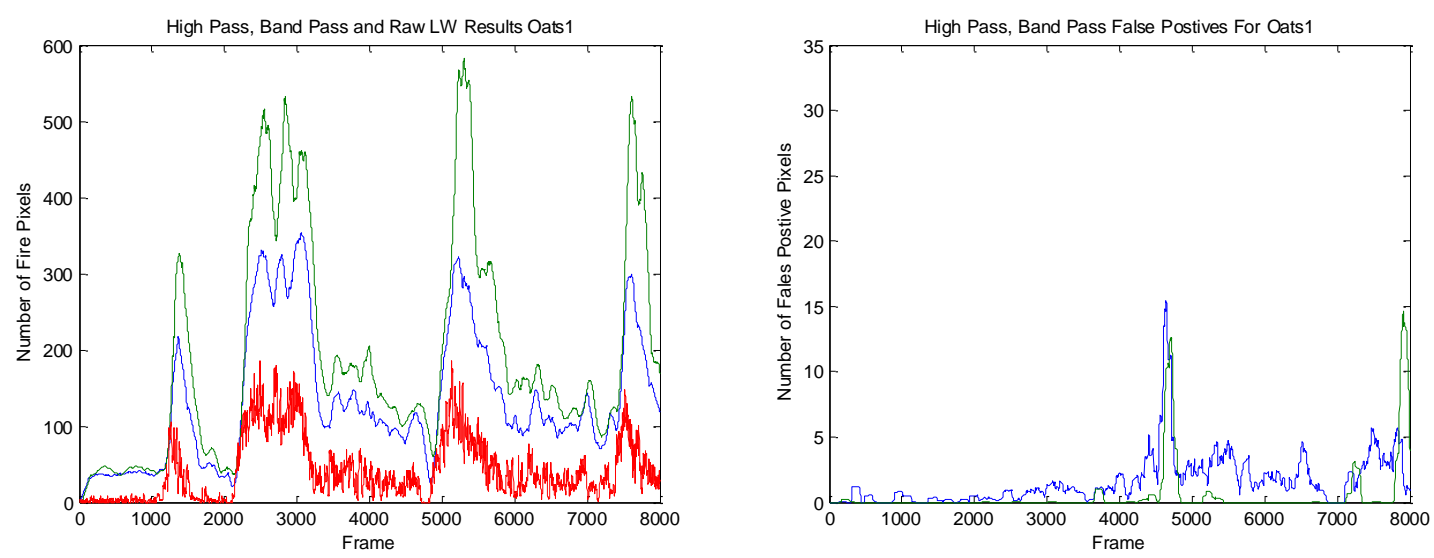

Figure 40 Oats1 Results, Band Pass is Green and High Pass is Blue

Frames 1 through 1080 display a small fire that is barely detectable using raw long wave data. The raw long wave data recorded on average 2.5 pixels of fire during this time. The band pass and high pass filtered data preform comparably and records on average 40 pixels of fire during the same time period. Due to the group delay of the filter the band pass result doesn't register until frame 60 or 2 seconds for the band pass filter and frame 35 or 1 second for the high pass filter.

During times of peak fire activity, frames 2200 through 3300, the band pass filter significantly out preforms the high pass filter. The band pass filter registers on average 450 fire pixels while the high pass registers on average 300 fire pixels.

\subsection{Examining False Positives}

The algorithm in this thesis is designed assuming no knowledge of where the center of the fire is. If implemented additional parameters can be added depending on the particular scene the camera is looking at. In general this algorithm is designed to look for forest fires away from civilization. In this setting most of the false positives due to traffic 
movement and buildings would not apply. Regardless these false positives still need to be looked at. Every instance where the 30 frame filtered false positive peaks above 25 pixels is recorded. Table 8 lists the total number of false positives for each data set until the frame rate started to fail.

Table 8 Total Number of False Positives for Each Data Set

\begin{tabular}{|l|l|l|l|l|}
\hline Data Set & Total Frames & $\begin{array}{l}\text { High Pass False } \\
\text { Positives }\end{array}$ & $\begin{array}{l}\text { Band Pass } \\
\text { False Positives }\end{array}$ & Comments \\
\hline Setup1 & 3753 & 0 & 0 & \\
\hline Charcoal1 & 4000 & 1 & 1 & \\
\hline Oats1 & 8000 & 1 & 2 & \\
\hline Oats2 & 395 & 0 & 0 & 2 \\
\hline Oats2b & 8000 & 2 & 2 & \\
\hline Oats3 & 8000 & 2 & 6 & $\begin{array}{l}\text { Frame rate } \\
\text { starts to fail }\end{array}$ \\
\hline Pine_needles & 14934 & 3 & 2 & \\
\hline Pine_cones & 8000 & 2 & 3 & $\begin{array}{l}\text { Frame rate } \\
\text { failed }\end{array}$ \\
\hline Palm & 4405 & 0 & N/A & \\
\hline Thistle & 6670 & N/A & & \\
\hline $\begin{array}{l}\text { The frame rate continues to deteriorate for the remaining data sets. False positive } \\
\text { readings are meaningless for the rest of the results }\end{array}$ &
\end{tabular}

There are several types of false positives. Common false positives get generated by initial movement frames, excessive random movement and high altitude signals. Most 
peak false positive readings correspond to multiple occurrences in separate location is the frame.

\subsubsection{Initial Movement Frames}

The first or last movement of an object occasionally triggers a positive fire reading. The object appears in the long wave infrared image slightly before the mid wave image. Because the object is not present in the mid wave image the long wave power is considerable more. Frame 4580 and 4581 on the band pass implementation shows an example of this type of false positive in Figure 41.
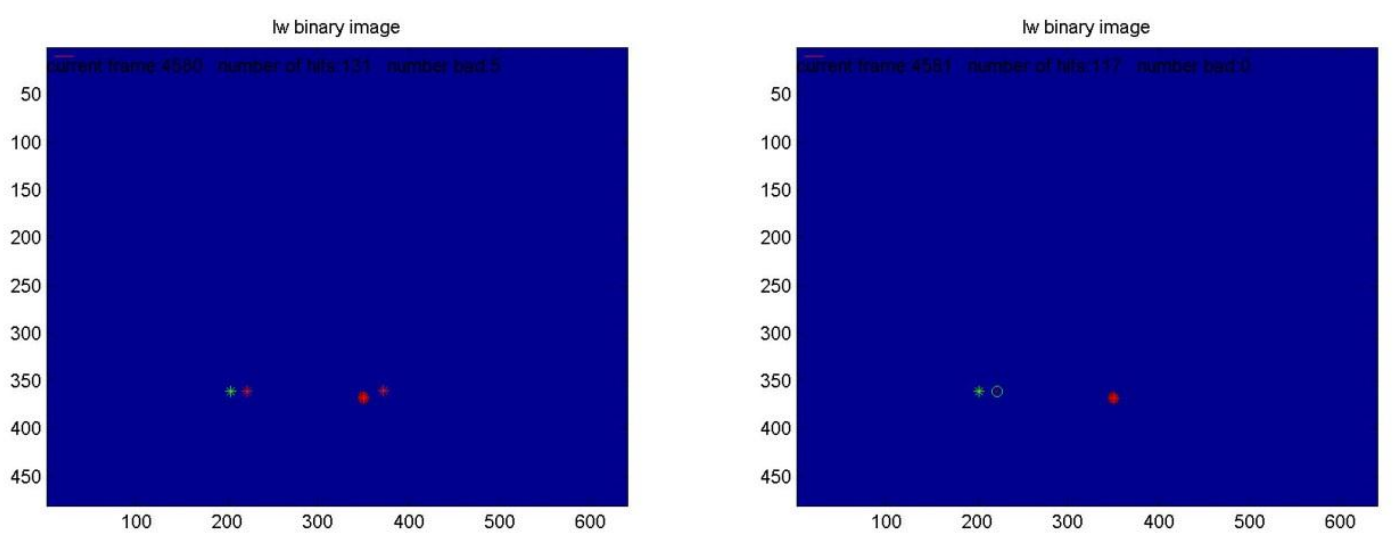

Figure 41 Example of false positive due to movement

\subsubsection{High Altitude False Positive}

The third false positive in the oats1 data set around frame 7921 appears well above the horizon in the upper left corner, see Figure 42. This point cannot be a fire even though there is 300 times as much power in the long wave over the mid wave. This object appear on and off for about 30 frames without moving. Similar appearing objects appear in this same location in later data sets. This must either be something in space or 
at a high altitude reflecting or generating long wave infrared energy. Another possibly is the pixels produces false measurements. Regardless the algorithm can be adjusted to ignore anything above the horizon.

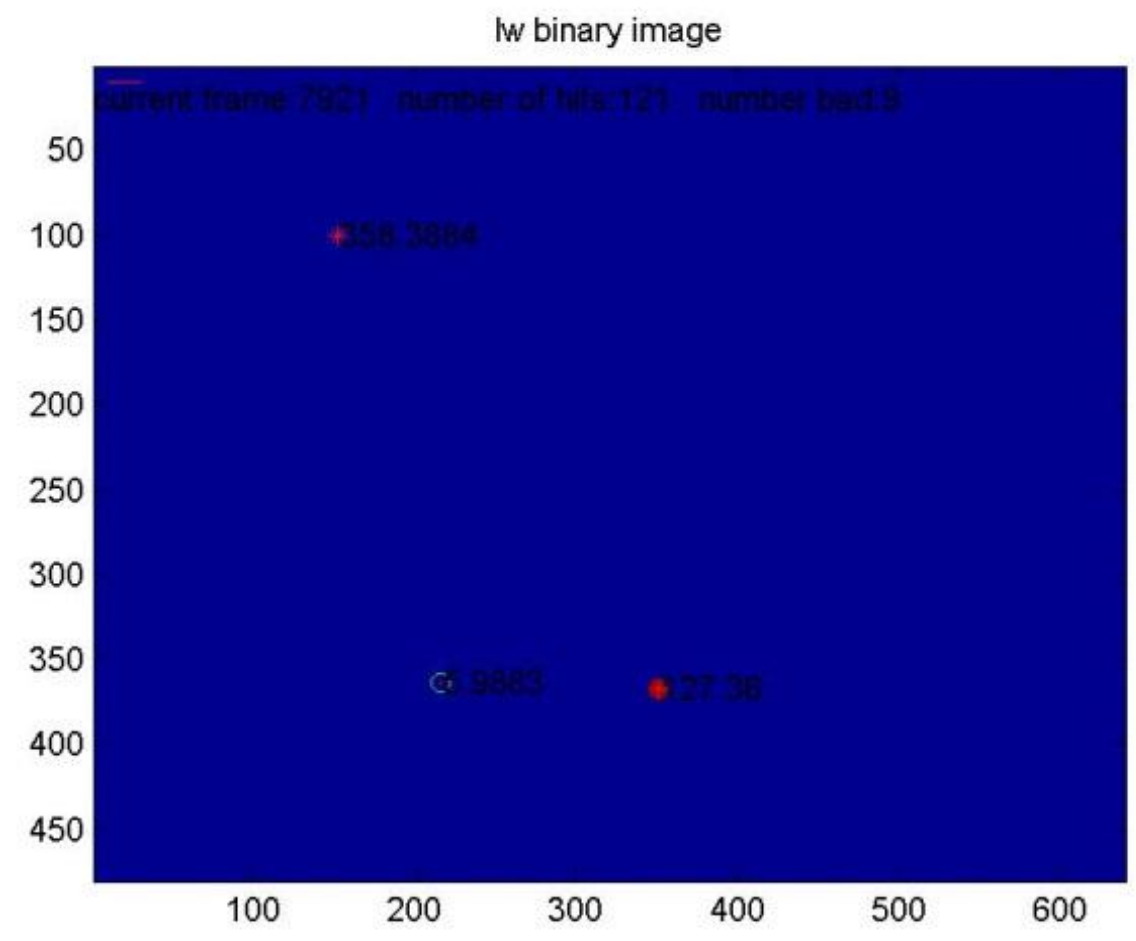

Figure 42 False Positive due to High Altitude Source

\subsubsection{False Positive due to Street Light}

Figure 43 shows another type of false positive appears to be location orientated. The pixels around location $(375,350)$ generate a large number of false positives when moving vehicles pass. On closer review a street light shines a light form about this location. This light must add some long wave energy without the corresponding mid wave energy to trigger a false positive. In an actual implementation areas around stationary lights like this may need additional filtering. 
IW binary image

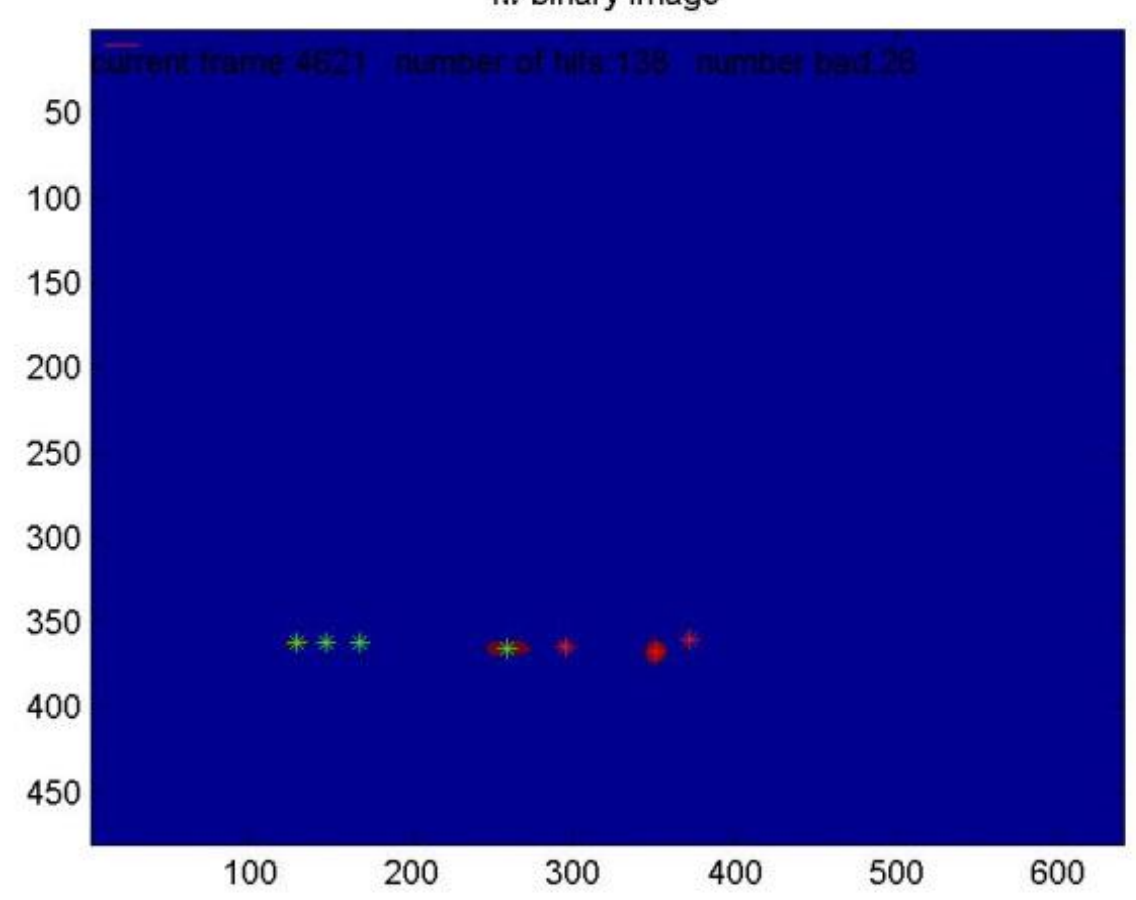

Figure 43 False Positive due to Street Light 


\section{CHAPTER 8}

\section{Proposed Implementation}

The goal of this thesis is to derive algorithms that can be used to accurately detect early forest fires. The next step is to implement these in a real time system. The implementation of this in a real world scenario depends on many factors outside to scope of this thesis like: sensor type, sensor data bus, processor type and how the data get to a user. This section briefly discusses some possible implementation issues that need addressing in the future.

These algorithms were designed and tested using MATLAB on previously recorded data. MATLAB simplifies the design process at the cost of performance. Because of this the current implementation can never run in real time. The current design uses the flow shown in Figure 44 and 
Table 9 shows the current required time for each step in MATLAB. These times are an example and may change depending on how many objects need fire/no fire analysis.

\begin{tabular}{|c|c|c|c|}
\hline $\begin{array}{l}\text { Read in } \\
\text { MW/LW Data }\end{array}$ & $\begin{array}{l}\text { Preform } \\
\text { Filtering }\end{array}$ & $\begin{array}{l}\text { Generat } \\
\text { Figures }\end{array}$ & $\begin{array}{l}\text { Generate } \\
\text { Movie File }\end{array}$ \\
\hline
\end{tabular}

Figure 44 Algorithm Block Diagram 
Table 9 Algorithm Processing Time Requirements

\begin{tabular}{|l|l|}
\hline Step: & Time: \\
\hline Read in MWIR /LWIR Data & 0.1840 seconds \\
\hline Preform Filtering & 0.1402 seconds \\
\hline Generate Figures & 0.0713 seconds \\
\hline Generate Movie File & 0.4165 seconds \\
\hline \hline Total & 0.8120 seconds \\
\hline & \\
\hline $\begin{array}{l}\text { Maximum Allowed Time } \\
\text { Per Frame (30 fps) }\end{array}$ & 0.0333 seconds \\
\hline
\end{tabular}

In the current implementation the algorithm uses 24 times that amount of time allowed to perform all steps. If only considering the filtering step the algorithm uses just over 4 times the maximum allowed time. MATLAB as an interpretive language cannot implement this algorithm in real time.

There are a couple of solutions to this, first is to use a compiled language like $\mathrm{C} / \mathrm{C}++$ instead of an interpretive one. Compilers convert code into a machine code once while optimizing the entire program. An interpreter, like MATLAB, must perform this action on each line of code as it run. Compiled code usually runs much quicker than interpreted code [25]. Using a compiled program alone should speed up the algorithm enough for real time use. 
A second solution, if just using compiled code doesn't gain enough performance, is to perform the operations in parallel using a graphical processing unit (GPU) [26]. Almost every step in the filtering can be optimized to work in parallel. A GPU can process every pixel of a frame independently and at the same time. The pixels require no information about their neighbors until the spatial filtering stage. The only step that requires a serial process is the connected component analysis but there is currently research being done on parallel implementations [27]. A parallel implementation should meet the 30 frames per second requirement. This parallel implementation requires significant changes in code for best optimization. 


\section{CHAPTER 9}

Future Work

The data analyzed in this thesis represents a very small and select range of fire data. The data analyzed always looks at the same scene, same fire location with the same environmental conditions. To truly create a robust fire detection system requires the analysis of additional variables. The variable that needs the most attention is the amount of ambient light. All of the fire data gathered took place during the middle of the day. Any fire detection system needs to have 24 hour coverage; forest fires don't only occur during the day. The cameras used for this thesis, MWIR and LWIR, were selected over the RBG camera for their performance in night time conditions. Using these cameras at night should yield similar results but this needs testing and verification.

Another concern involves the distance of the fire. As discussed in chapter 2 the atmosphere absorbs LWIR and MWIR signals differently. The algorithm may misclassify a very close fire and a further away fire due to the increased mid wave energy the sensor receives. As the fire moves further away the sensor receives less energy from both bands. Both of these conditions need testing.

Additionally neither this algorithm nor any others designed off these data sets can run on a real time system in their current implementation. The purpose of this thesis was to find algorithms the can detect forest fire. Implementing them in a real time system is a different project full of its own challenges. In order for this algorithm ever to detect a forest fire it needs to be adapted to a real time system. 


\section{References}

[1] J.R. Martinez-de Dioa; T.A. Ollero; B.C. Arrue; "Distributed Intelligent Automatic Forest-Fire Detection System," Presented at INNOCAP Conference, Grenoble, France, 1999.

[2] "CAL FIRE 2011 Wildland Fire Summary", Internet:

http://calfire.ca.gov/communications/downloads/fact_sheets/2011Summary_web.pdf, April 2012 [May 23, 2013].

[3] "CAL FIRE 2007 Wildland Fire Summary", Internet:

http://calfire.ca.gov/communications/downloads/fact_sheets/2007Summary_web.pdf, Sept 2011 [May 23, 2013].

[4] "OneEarth Southern Califorina Fire Images", Internet:

http://onearth.jpl.nasa.gov/socalfires/, October 25, 2007 [May 23, 2013]

[5] Y. G. Sahin and T. Ince. Early Forest Fire Detection Using Radio-Acoustic Sounding System. Sensors. 2009; 9(3):1485-1498.

[6] "Smoke Alarms" Internet:

http://www.fire.ca.gov/communications/downloads/fact_sheets/SmokeAlarms.pdf. Oct 2012, [May 23 2013]

[7] D. Stipanicev; T. Vuko; D. Krstinic; M. Stula; L. Bodrozic; "Forest Fire protection by advanced Video Detection System- Croatian Experiences," In Third TIEMS WorkshopImprovement of Disaster Management System. Trogir, 2006.

[8]Angayarkkani, K.; Radhakrishnan, N.; "Efficient Forst Fire Detection System: A Spatial Data Mining and Image Processing Based Approach," Computer Science and Network Security, 2009., IJCSNS International Journal of, vol. 9 , no. 9, pp. 100-106, Mar 2009

[9] "MODIS Website - Components of MODIS", Internet:

http://modis.gsfc.nasa.gov/about/specifications.php [May 29, 2013]

[10] Timothy M. Davenport; Early Forest Fire Detection Using Texture Analysis of Principal Components from Multispectral Video; Thesis, EE Department, Cal Poly, SLO, June 2012

[11] Daniel G. Kohler; Study of Statistical and Computational Intelligence Methods of Detecting Temporal Signature of Forest Fire Heat Plume from Single-Band GroundBased Infrared Video; Thesis, EE Department, Cal Poly, SLO, June 2012 
[12] Georges F. Moussa; Early Forest Fire Detection Using Texture, Blob Threshold, And Motion Analysis Of Principal Components; Thesis, EE Department, Cal Poly, SLO, December 2012

[13] R. C. Gonzalex and R. E. Woods, Digital Image Processing 3rd ed. New Jersey: Pearson Education Inc., 2008

[14] Gunapala, S.D.; Bandara, S. V.; Hill, C.J.; Liu, J.K.; Mumolo, J.M.; Keo, S.A., "Pixel co-registered simultaneous dualband infrared sensing," Sensors, 2008 IEEE , vol., no., pp. $1596,1599,26-29$ Oct. 2008

doi: $10.1109 / 2008.4716755$

[15] S. P. Urbanski, W. M Hao and S. Baker, "Chapter 4 Chemical Composition of Wildland Fire Emissions", in: Developments in Environmental Science Volume 8. 2009

[16] Han Sun, et. all. "Spectral characteristics of infrared radiation from forest fires." Proc. SPIE 6298, Remote Sensing and Modeling of Ecosystems for Sustainability III, 629808 (September 27, 2006); doi:10.1117/12.679775.

[17] “The Infrared Wall Chart” Internet: http://www.raytheon.com/ourcompany/rtnwcm/groups/ncs/documents/content/rtn_ncs_b usiness_rvs_wallchart.pdf Raytheon Vision Systems 2011 [May 23, 2013]

[18] R. G. Brown and P. Y. Hwang. Introduction to Random Signals and Applied Kalman Filtering $3^{\text {rd }}$ ed. New York: John Wiley \& Sons, 1997

[19] 1. L. Gaile and I. Radinsh, "Dynamic Response Of Tower Structures" Proceedings of the 8th International Scientific and Practical Conference. Volume I1 (2011)

[20] A. Ambardar. Analog and Digital Signal Processing 2nd ed. Brooks/Cole Publishing Company, 1999.

[21] R. Schaumann, H. Xiao and M. E. Van Valkengurg, Design of Analog Filters, 2nd ed. New York: Oxford University Press, 2010.

[22] D. A. Forsyth and J. Ponce, Computer Vision: A Modern Approch $2^{\text {nd }}$ ed. City : Prentice Hall, 2011

[23] G. X. Ritter and J. N. Wilson, Computer Vision Algorithms in Image Algebra New York: CRC Press 1996 
[24] Kyung Ah Han; Jong Chan Lee; Chi Jung Hwang, "Image clustering using selforganizing feature map with refinement," Neural Networks, 1995. Proceedings., IEEE International Conference on , vol.1, no., pp.465,469 vol.1, Nov/Dec 1995

[25] Matlab-to-C++ time-saver," Spectrum, IEEE , vol.34, no.4, pp.14,15, April 1997

[26] Hong, J.Y.; Wang, M.D., "High speed processing of biomedical images using programmable GPU," Image Processing, 2004. ICIP '04. 2004 International Conference on, vol.4, no., pp.2455,2458 Vol. 4, 24-27 Oct. 2004

[27] Mozef, E.; Weber, S.; Jaber, J.; Tisserand, E., "Parallel architecture dedicated to connected component analysis," Pattern Recognition, 1996., Proceedings of the 13th International Conference on, vol.4, no., pp.699,703 vol.4, 25-29 Aug 1996 
Appendices
A. Results for All Data Sets
B. MATLAB Code Used 


\section{APPENDIX A}

\section{Results for All Data Sets}

Table 10 shows the file name for the movie results generated from each data set using b_batch_run.m script.

Table 10 Results Movie Files

\begin{tabular}{|l|l|l|}
\hline Data Set & High Pass Movie File Name & Band Pass Movie File Name \\
\hline Applewood1 & dual_fir_highpass_applewood1.avi & dual_iir_bandpass_applewood1.avi \\
\hline Applewood2 & dual_fir_highpass_applewood2.avi & dual_iir_bandpass_applewood2.avi \\
\hline Charcoal1 & dual_fir_highpass_charcoal1.avi & dual_iir_bandpass_charcoal1.avi \\
\hline Extinguish & dual_fir_highpass_extinguish.avi & dual_iir_bandpass_extinguish.avi \\
\hline Oats1 & dual_fir_highpass_oats1.avi & dual_iir_bandpass_oats1.avi \\
\hline Oats2 & dual_fir_highpass_oats2.avi & dual_iir_bandpass_oats2.avi \\
\hline Oats2b & dual_fir_highpass_oats2b.avi & dual_iir_bandpass_oats2b.avi \\
\hline Oats3 & dual_fir_highpass_oats3.avi & dual_iir_bandpass_oats3.avi \\
\hline Palm & dual_fir_highpass_palm.avi & dual_iir_bandpass_palm.avi \\
\hline Pine_cones & dual_fir_highpass_pine_cones.avi & dual_iir_bandpass_pine_cones.avi \\
\hline Pine_needles & dual_fir_highpass_pine_needles.avi & dual_iir_bandpass_pine_needles.avi \\
\hline Rosemary & dual_fir_highpass_rosemary.avi & dual_iir_bandpass_rosemary.avi \\
\hline Setup1 & dual_fir_highpass_setup1.avi & dual_iir_bandpass_setup1.avi \\
\hline Thistle & dual_fir_highpass_thistle.avi & dual_iir_bandpass_thistle.avi \\
\hline Wet_dry1 & dual_fir_highpass_wet_dry1.avi & dual_iir_bandpass_wet_dry1.avi \\
\hline Wet_dry2 & dual_fir_highpass_wet_dry2.avi & dual_iir_bandpass_wet_dry2.avi \\
\hline Wet_leaves & dual_fir_bandpass_wet_leaves.avi & dual_iir_bandpass_wet_leaves.avi \\
\hline
\end{tabular}


Each movie file displays six different frames. From top left to bottom right displays the following:

Top left: Filtered MWIR Power Image

Top right: Threshold of filtered MWIR Image (not used, for reference only)

Middle left: Filtered LWIR Image

Middle right: Threshold of Filtered LWIR image (final results)

Bottom left: Raw LWIR Power Image

Bottom Right: Threshold of LWIR Image

The threshold of LWIR image frame includes three different markers one for each detected object explained in Table 11.

Table 11 Detected Object Markers

\begin{tabular}{|l|l|}
\hline Symbol & Object Type \\
\hline Red $*$ & Fire Object \\
\hline Green $*$ & Non-Fire (Failed Eccentricity test) \\
\hline Green O & Non-Fire (Failed LW vs. MW Power test) \\
\hline
\end{tabular}

The rest of the charts in this appendix display the total fire results and false positives for each data set. 


\section{Setup1:}
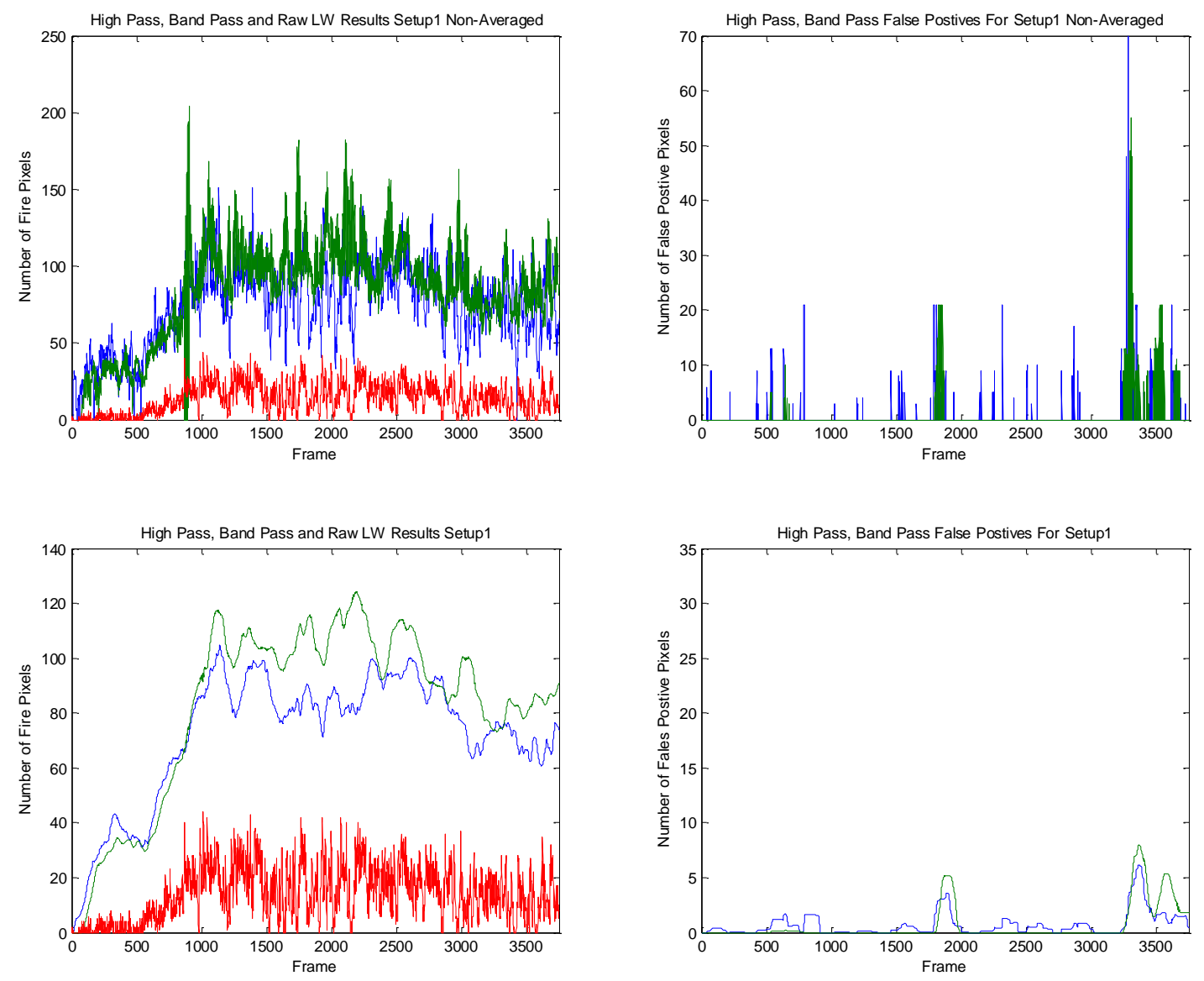

\section{Charcoal1:}
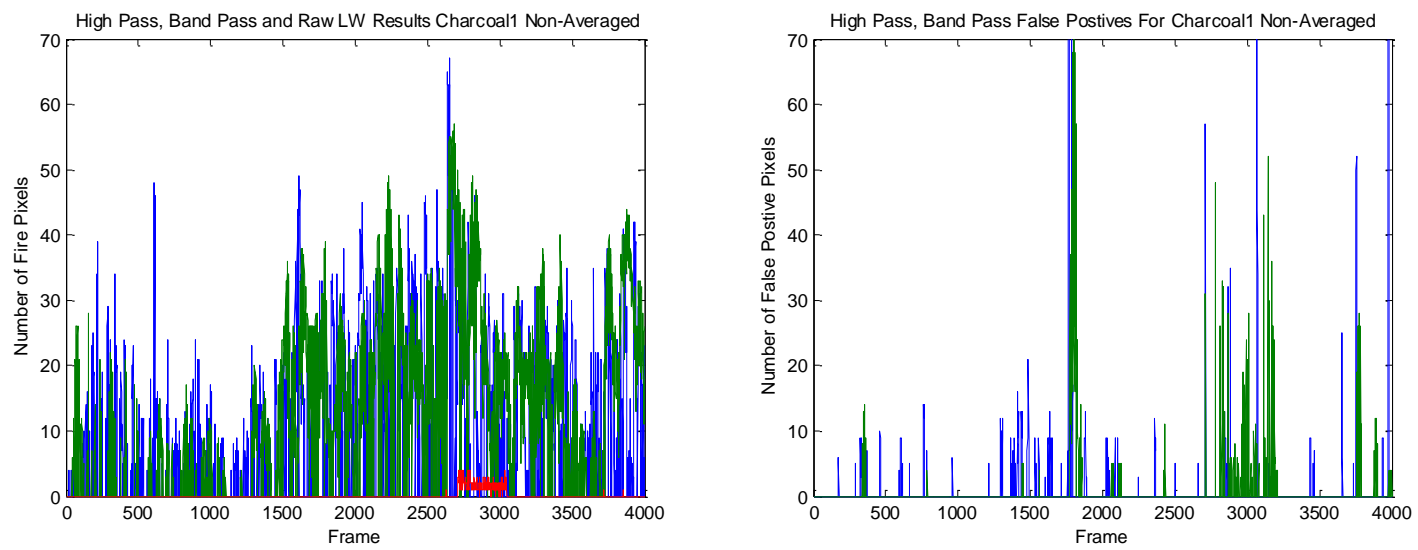

Page 68 

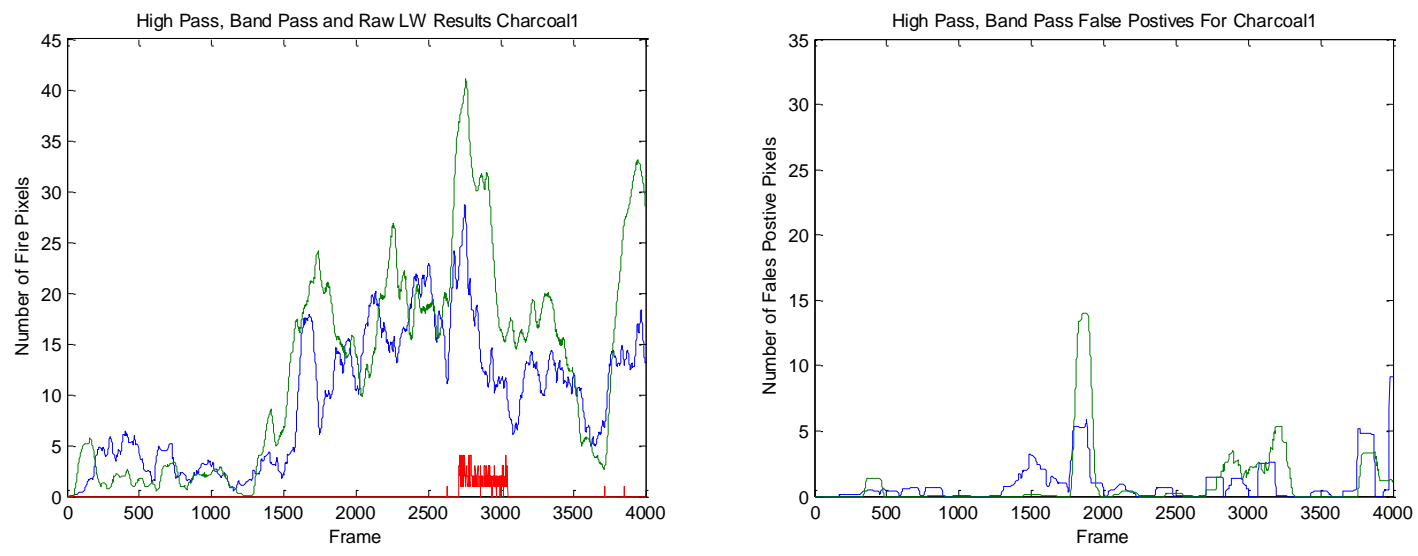

\section{Oats 1:}
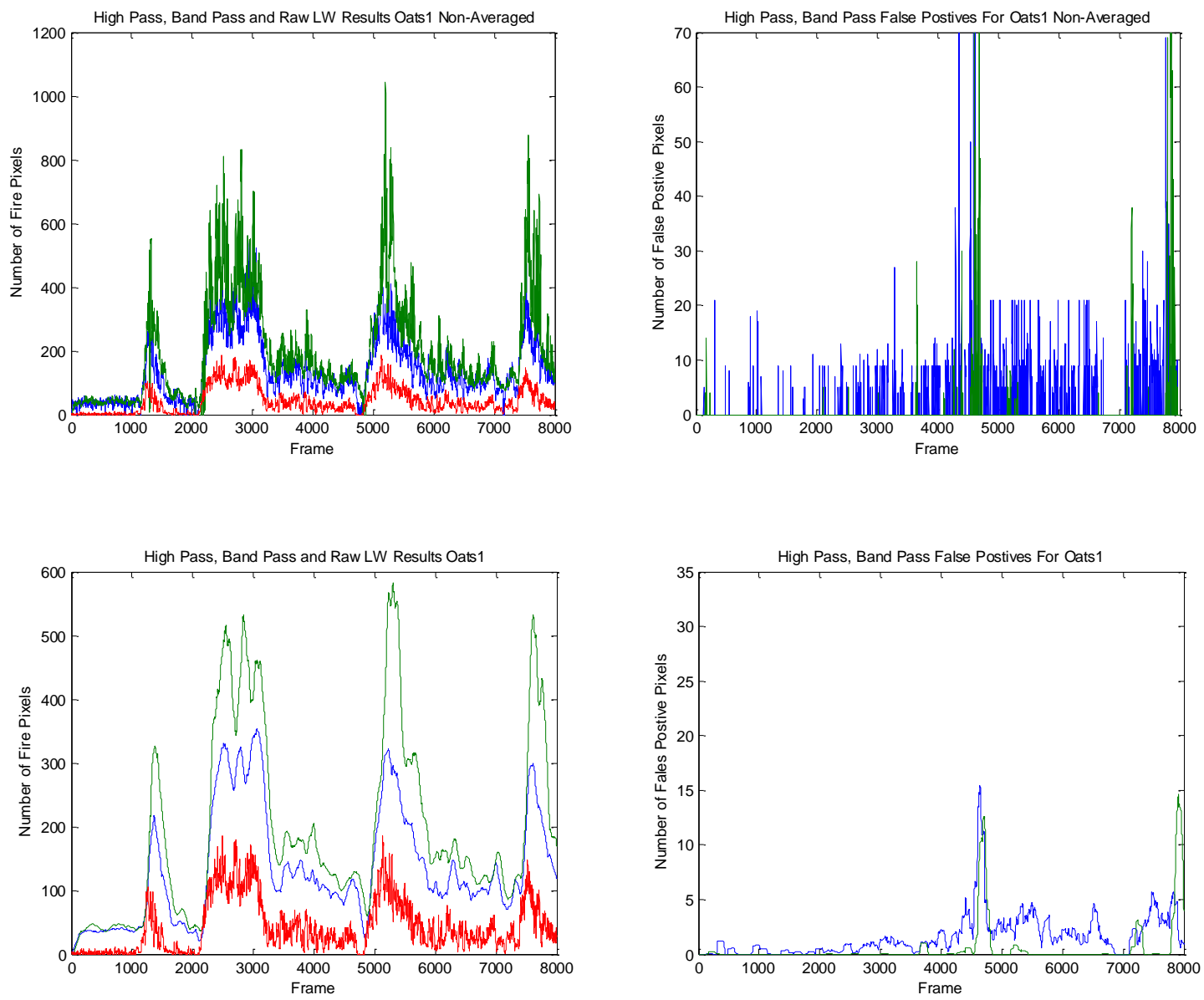

\section{Page 69}




\section{Oats2:}
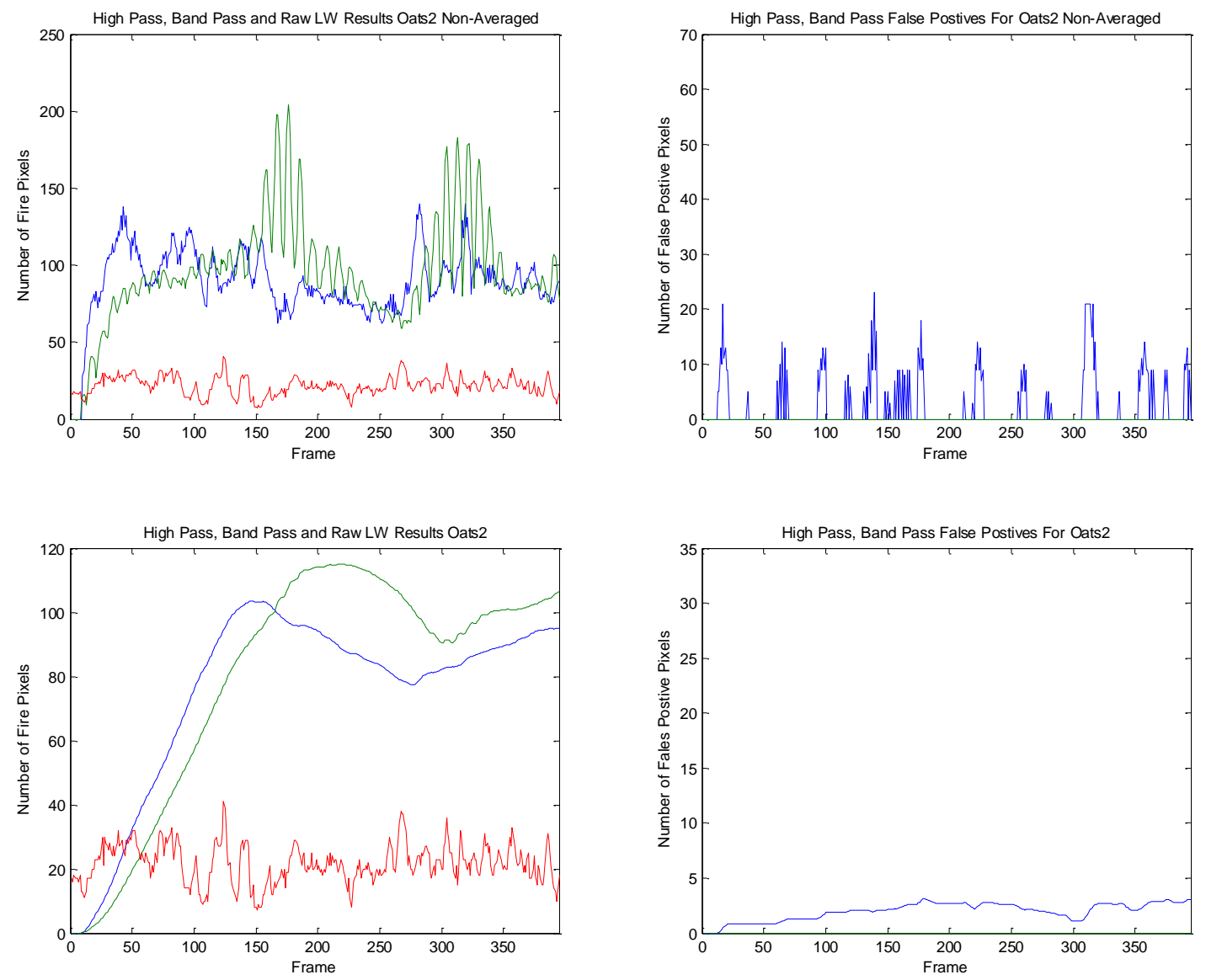

\section{Oats2b:}
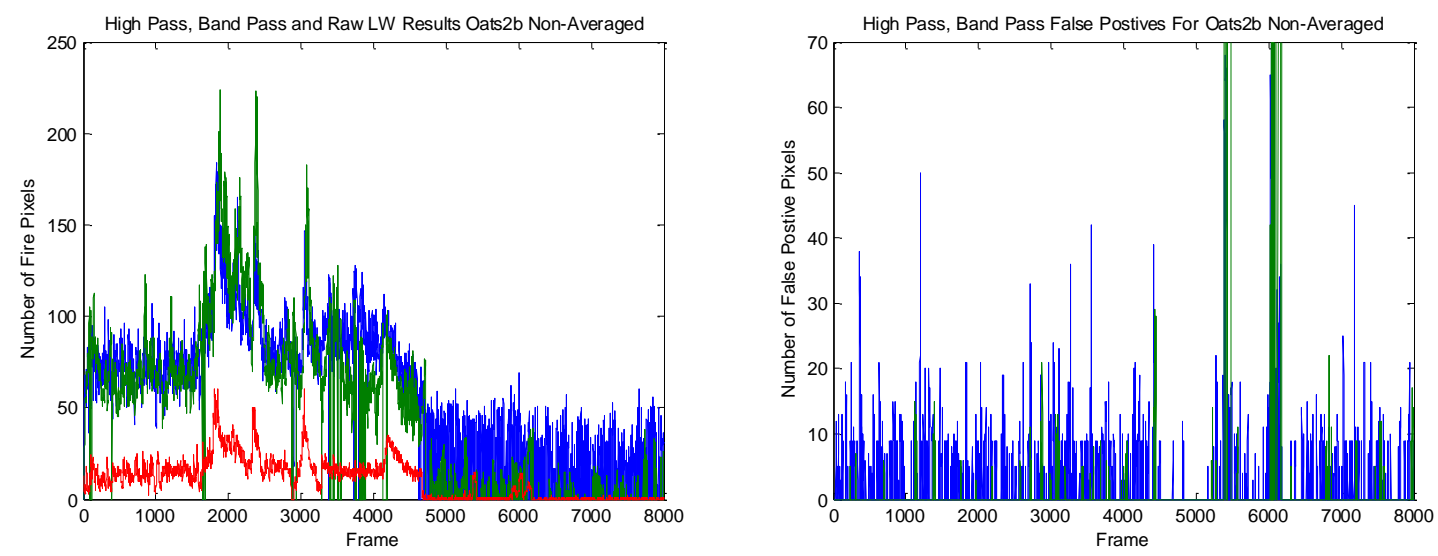

Page 70 

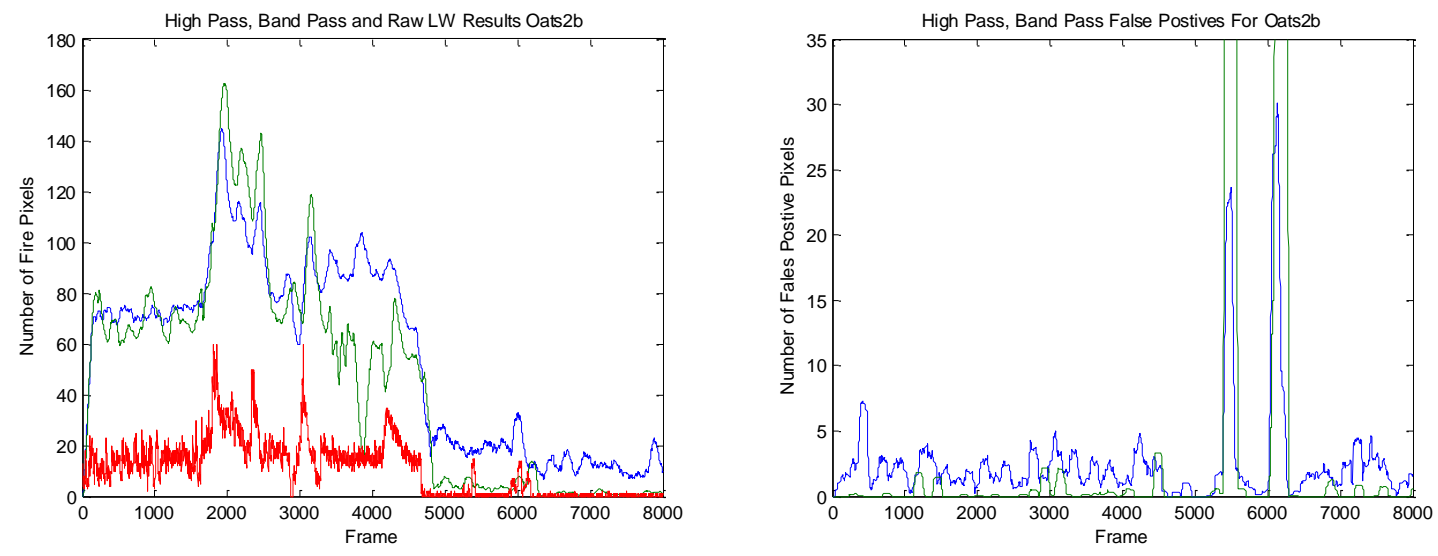

\section{Oats3:}
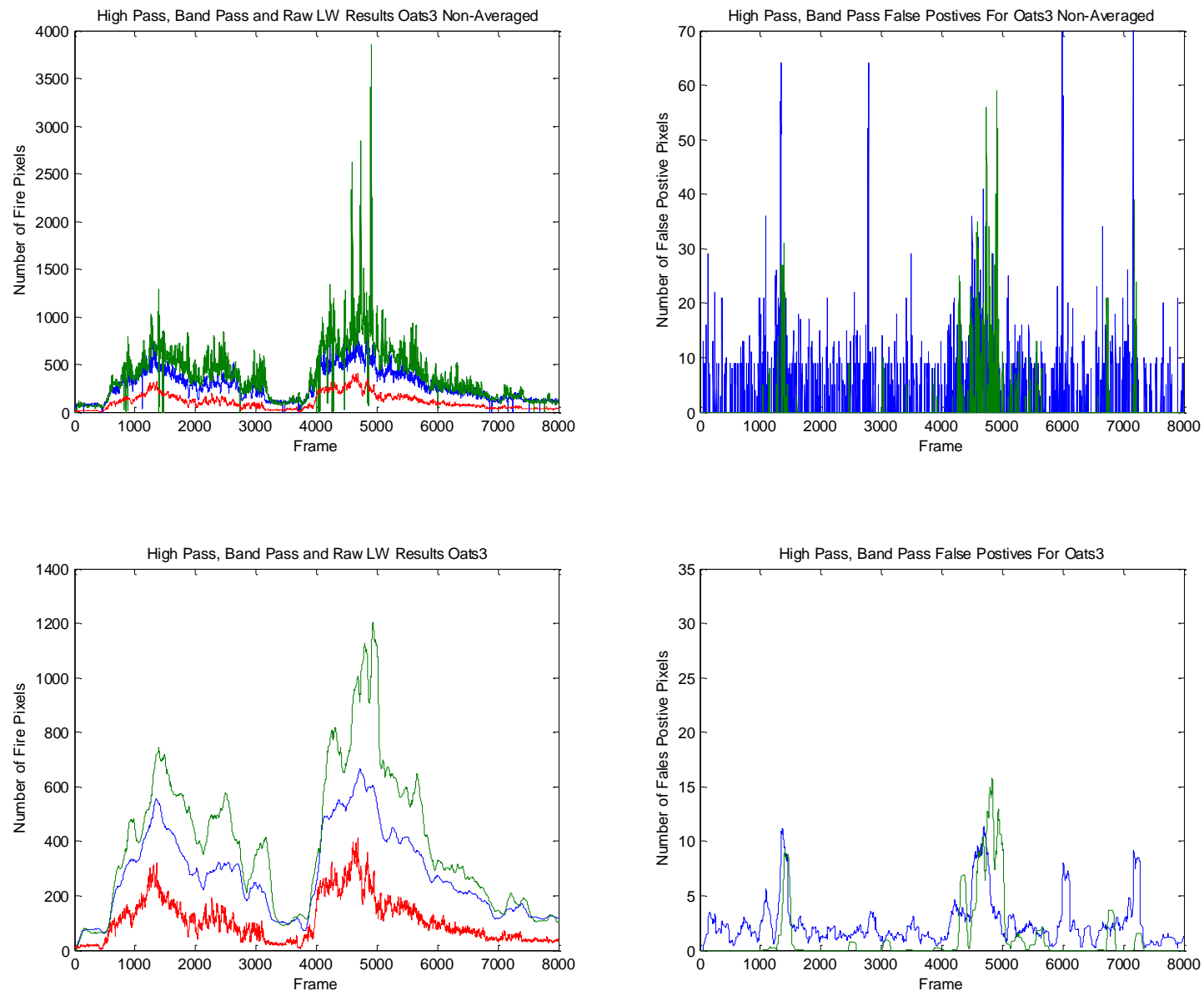

Page 71 
Pine Needles:
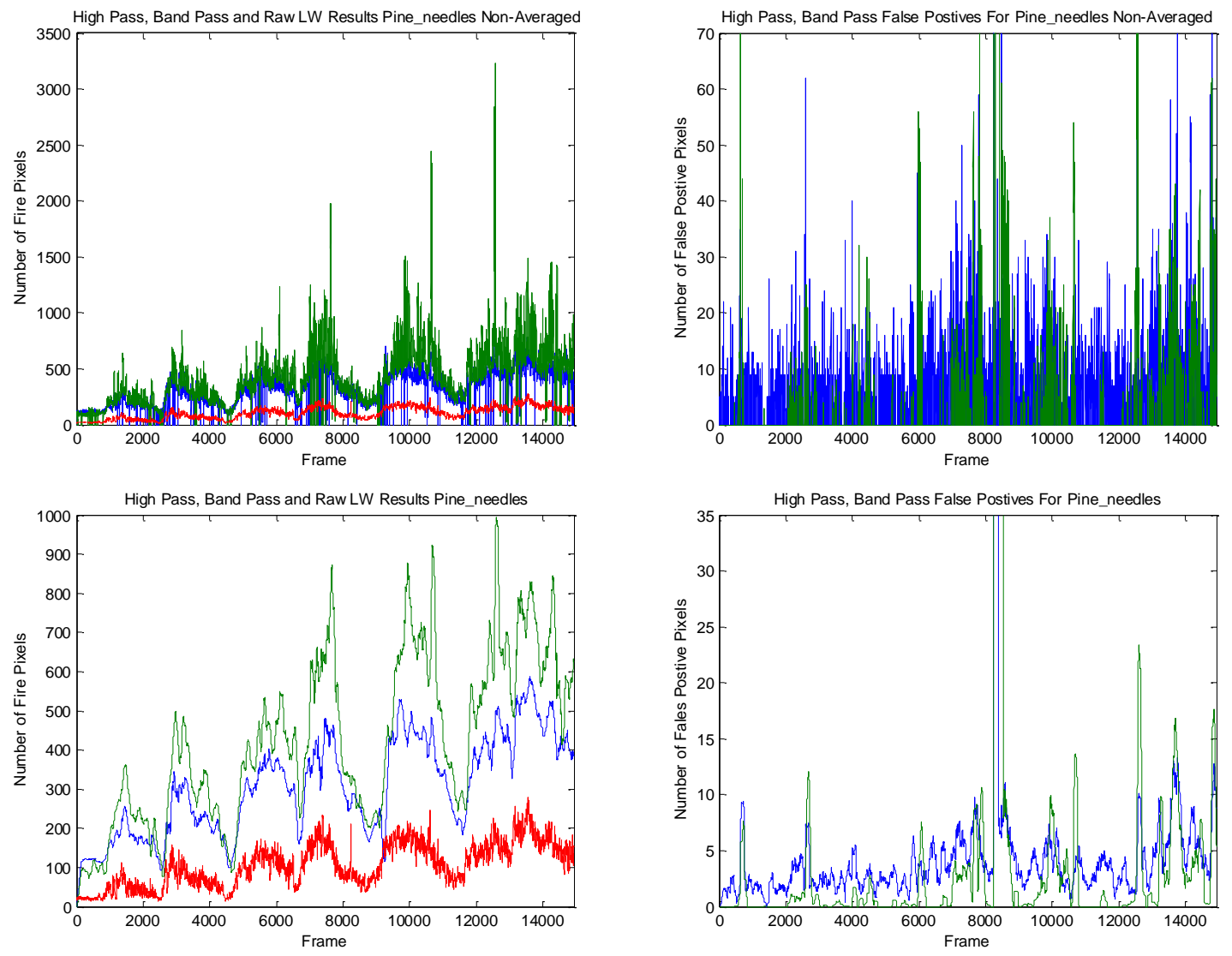

\section{Pine Cones:}
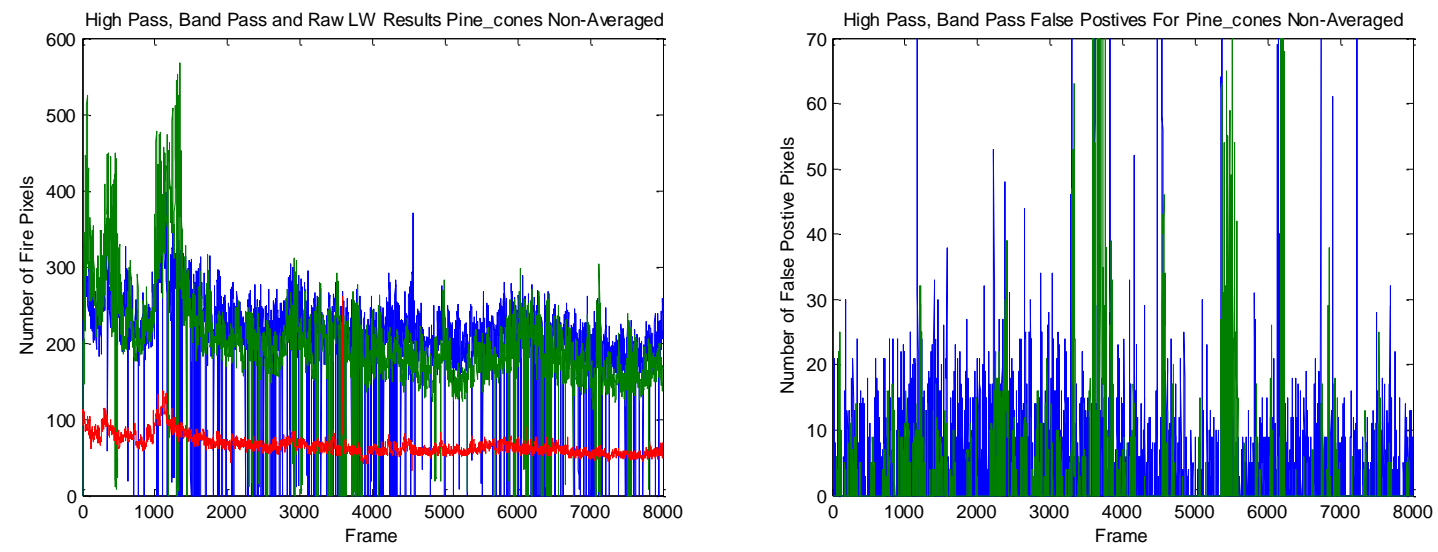

Page 72 

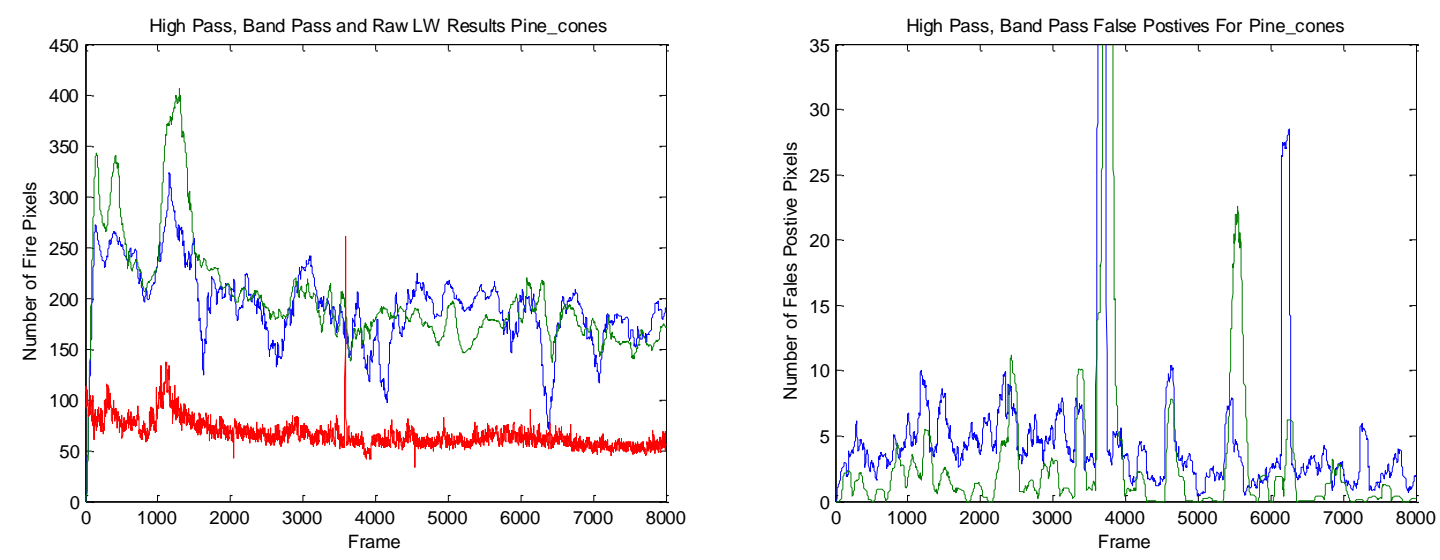

\section{Palm:}
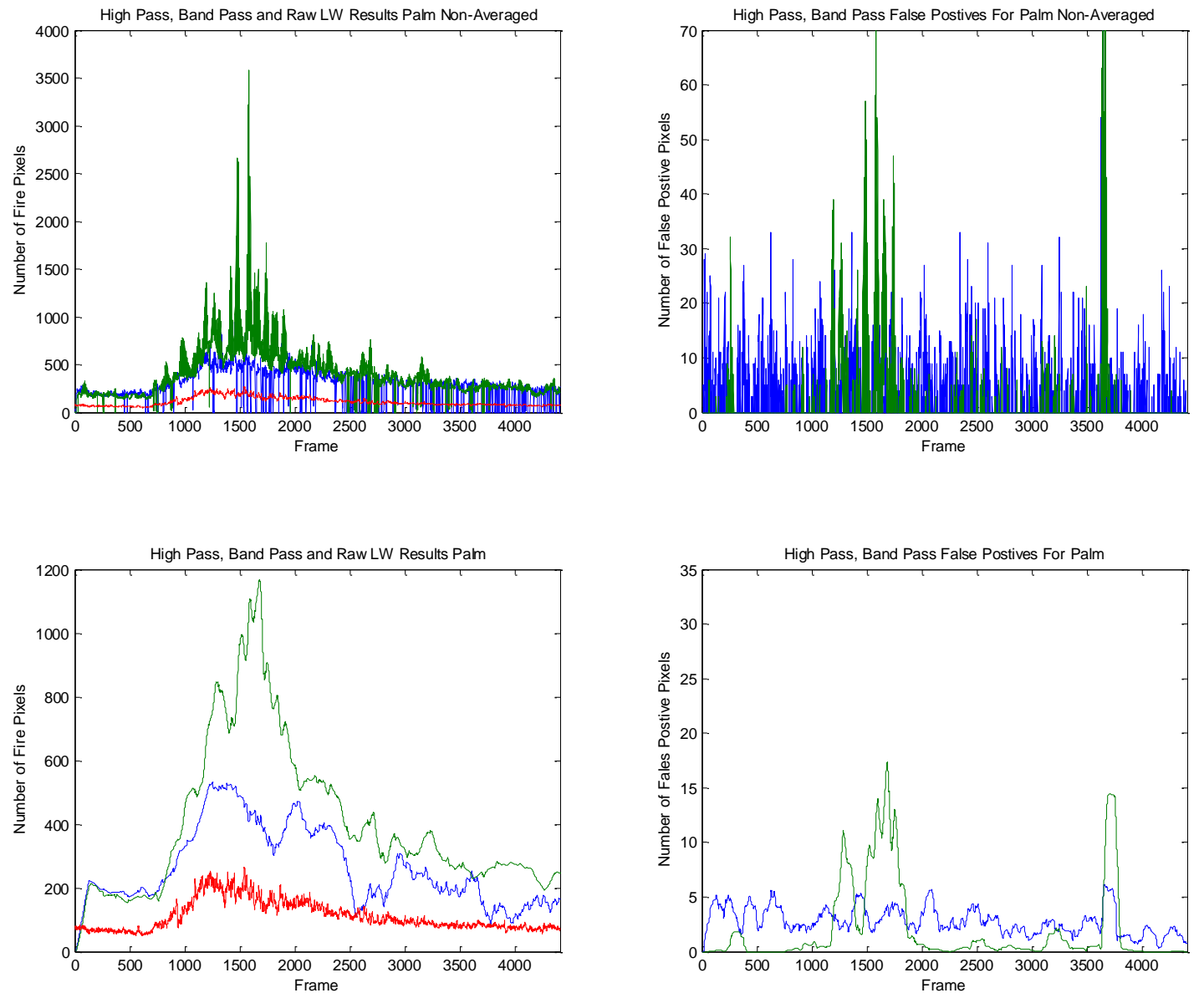

Page 73 


\section{Thistle:}
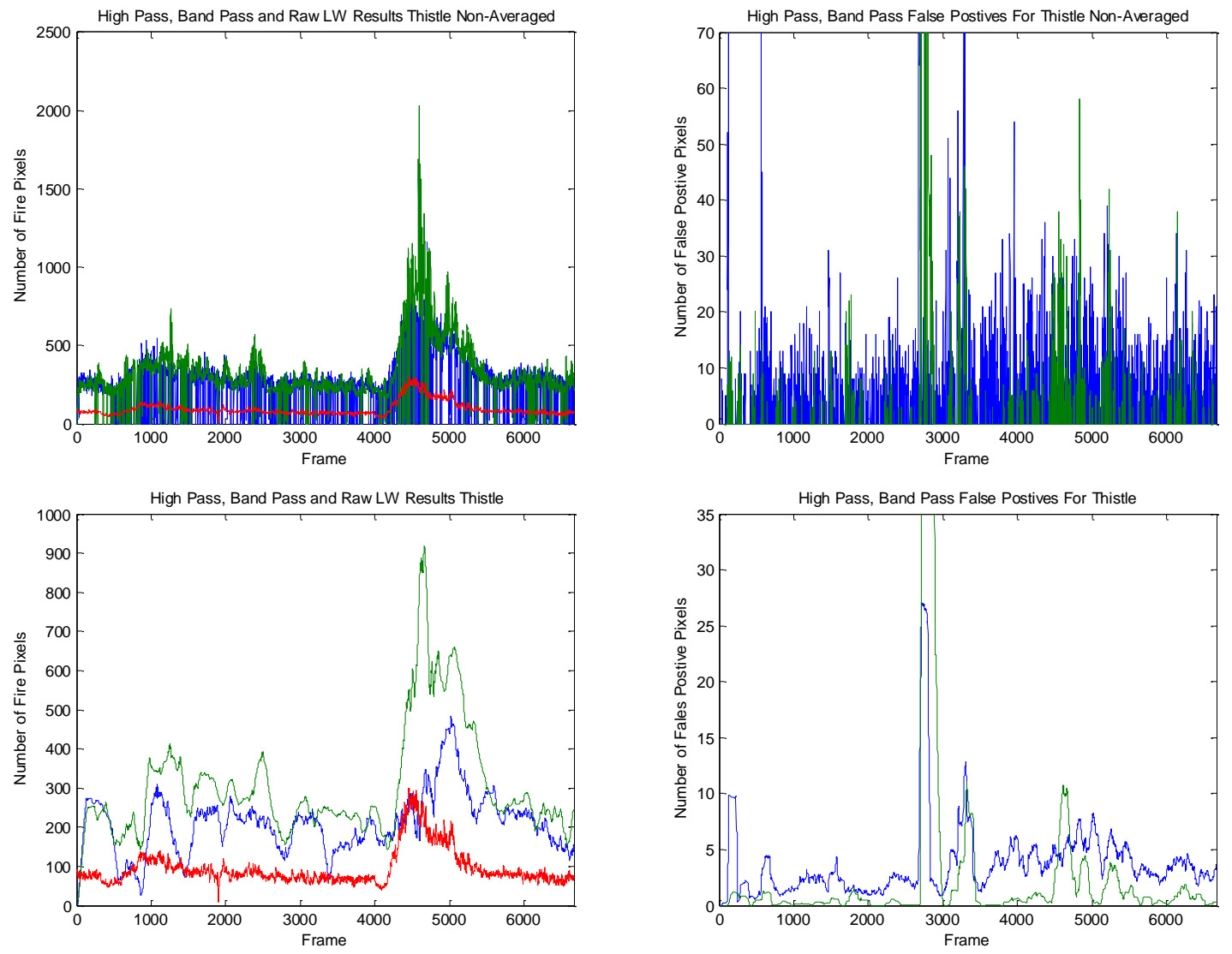

\section{Wet_leaves:}
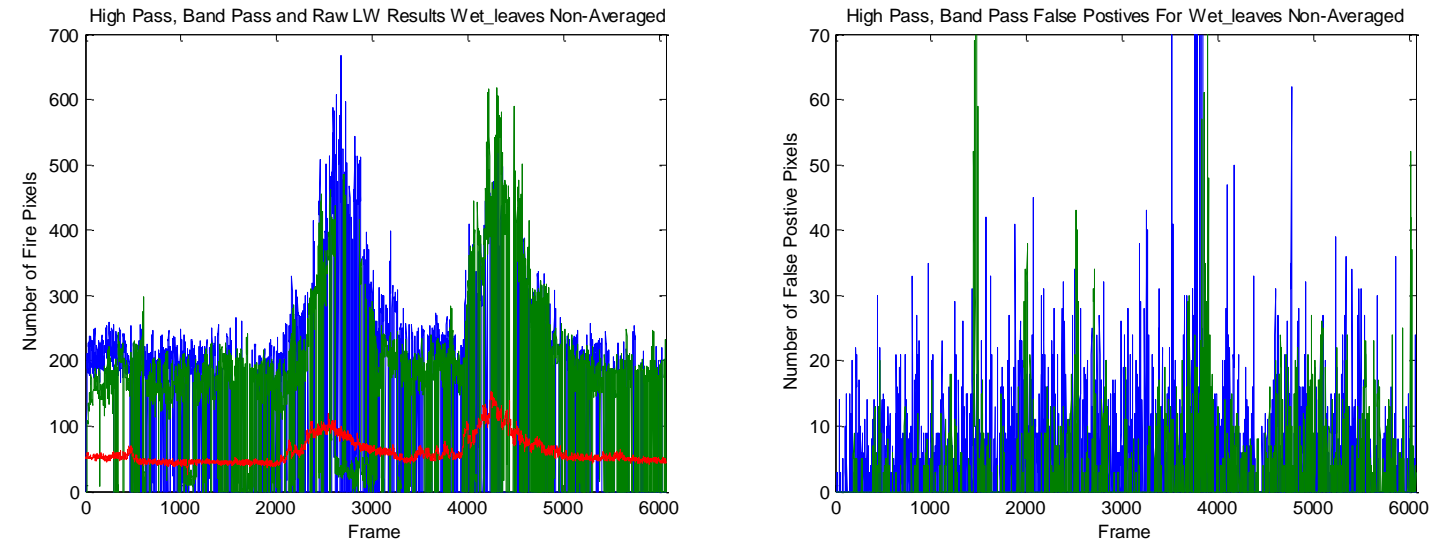

Page 74 

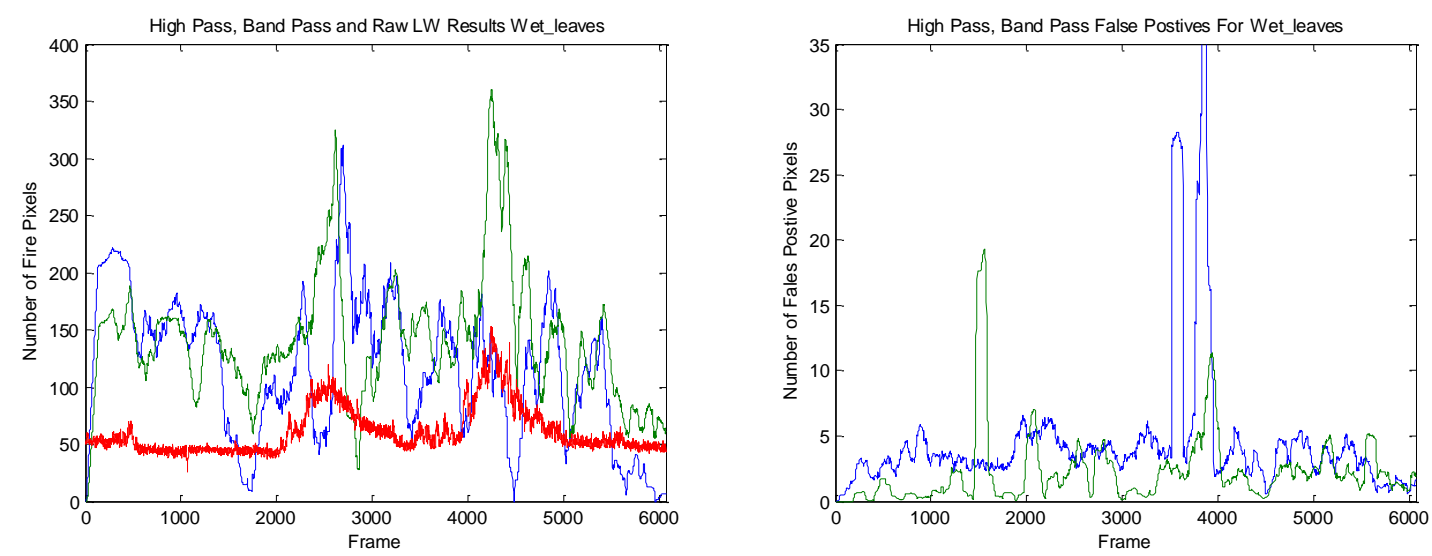

Wet_dry1:
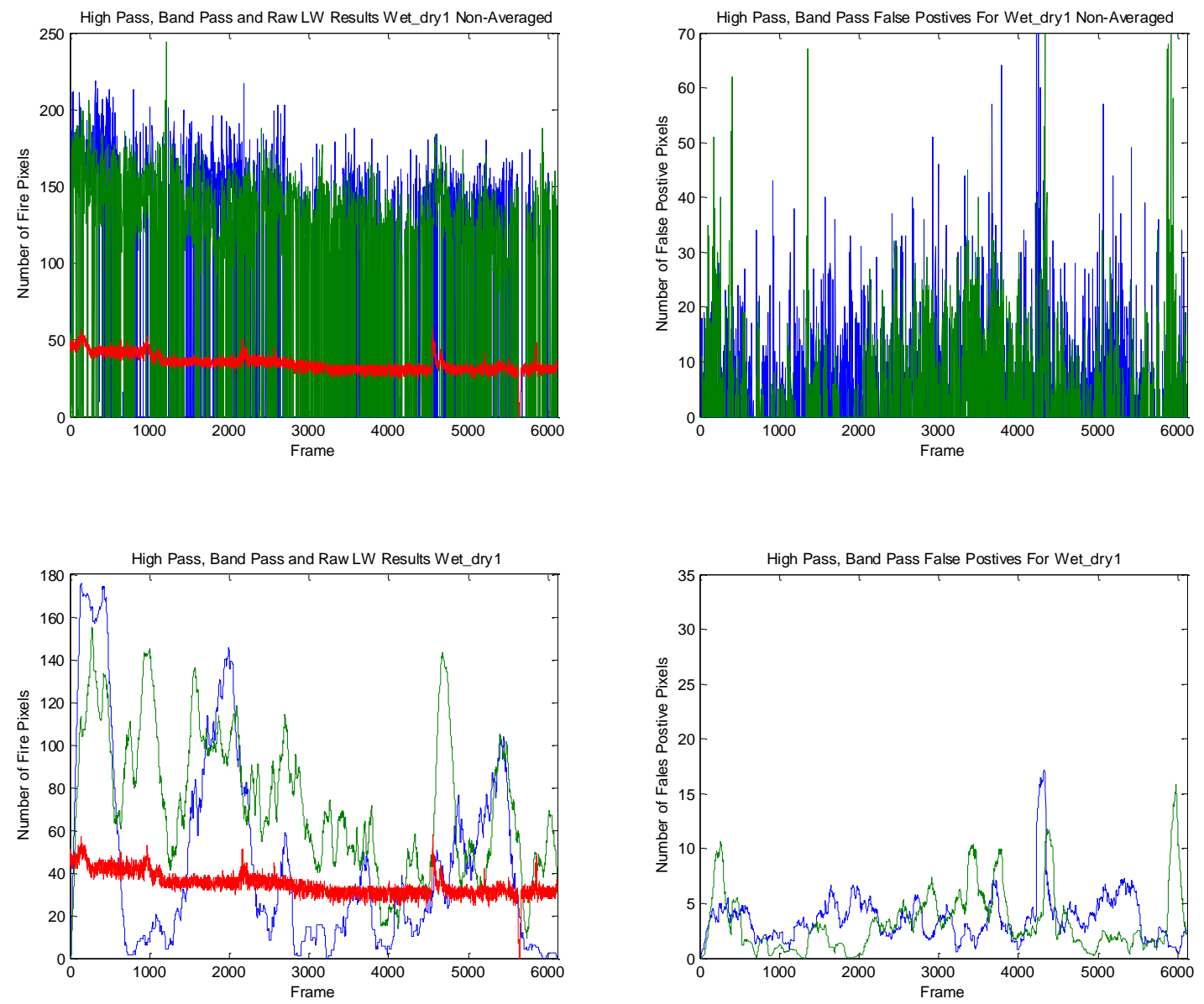

Page 75 


\section{Wet_dry2:}
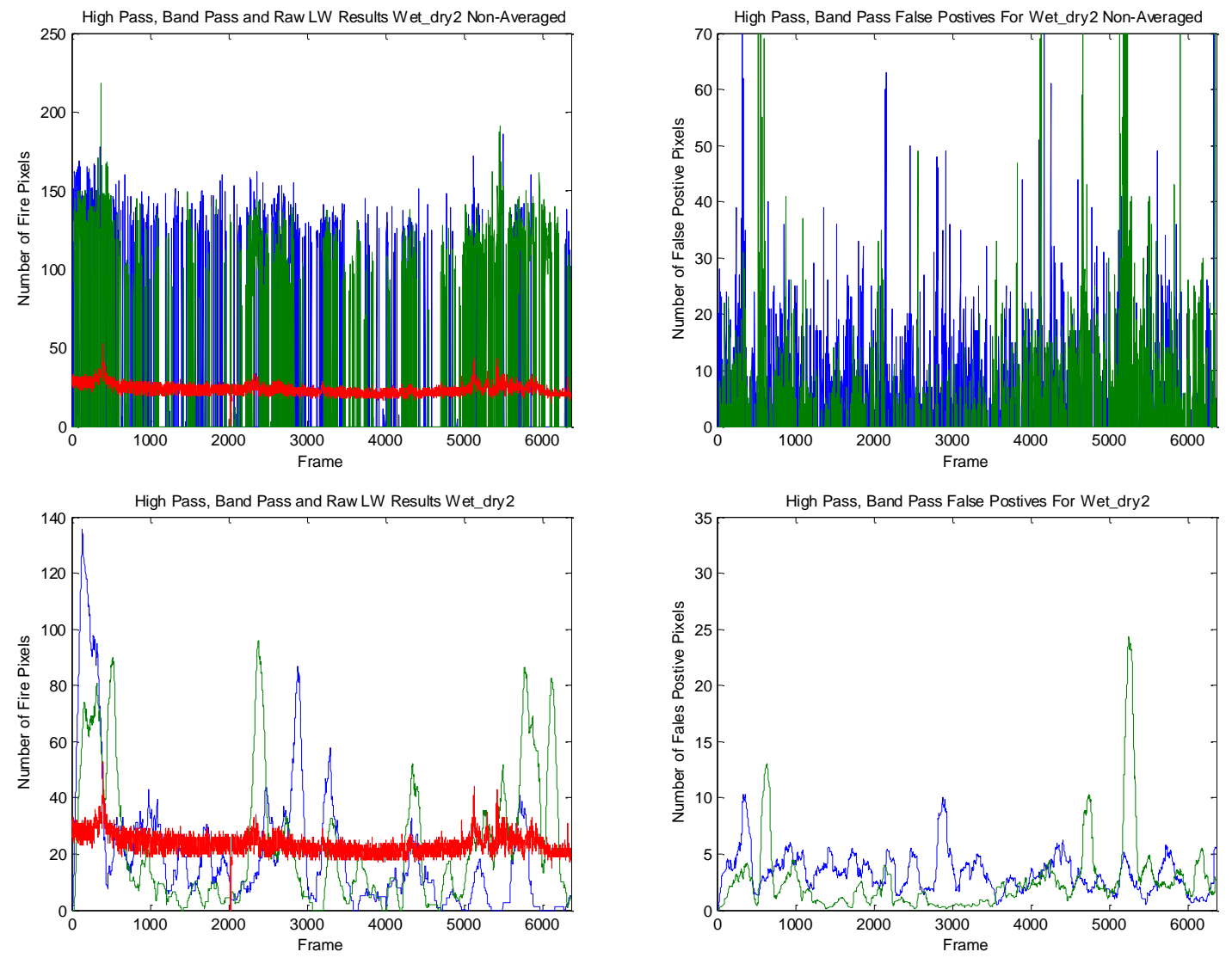

\section{Rosemary:}
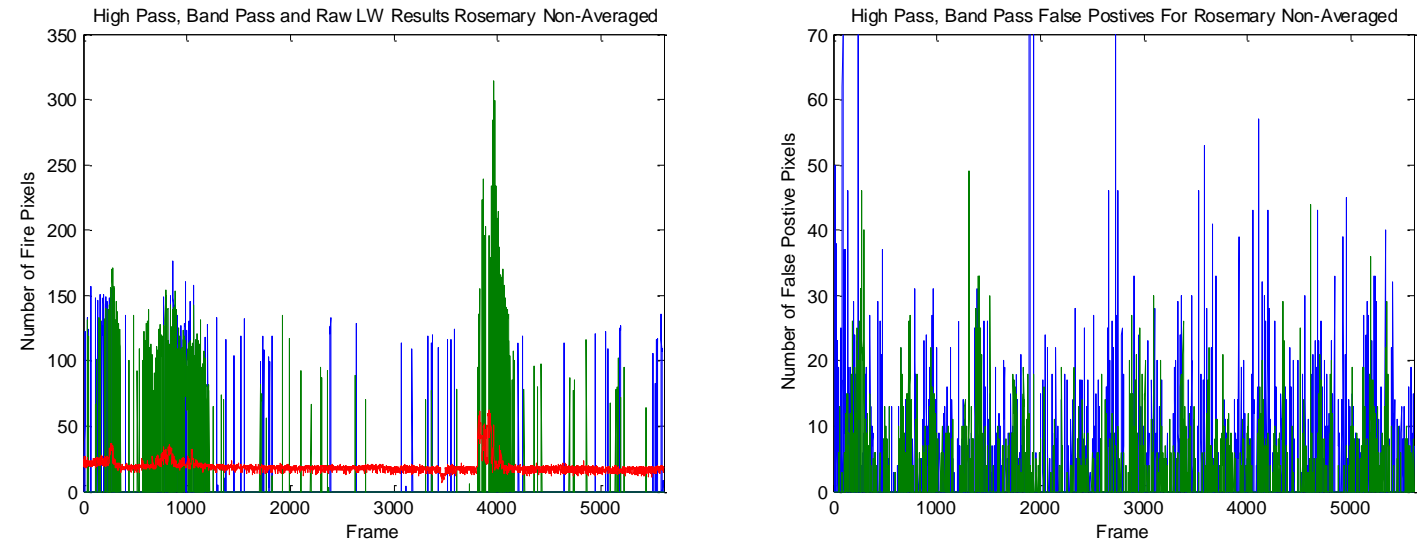

Page 76 

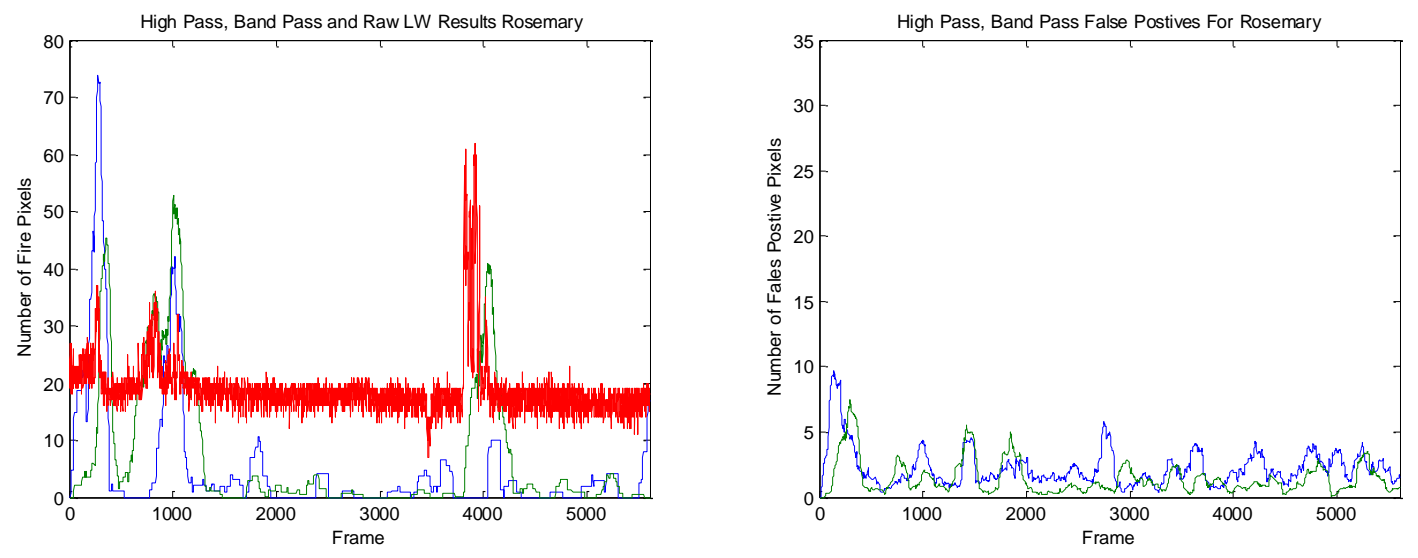

\section{Applewood1:}
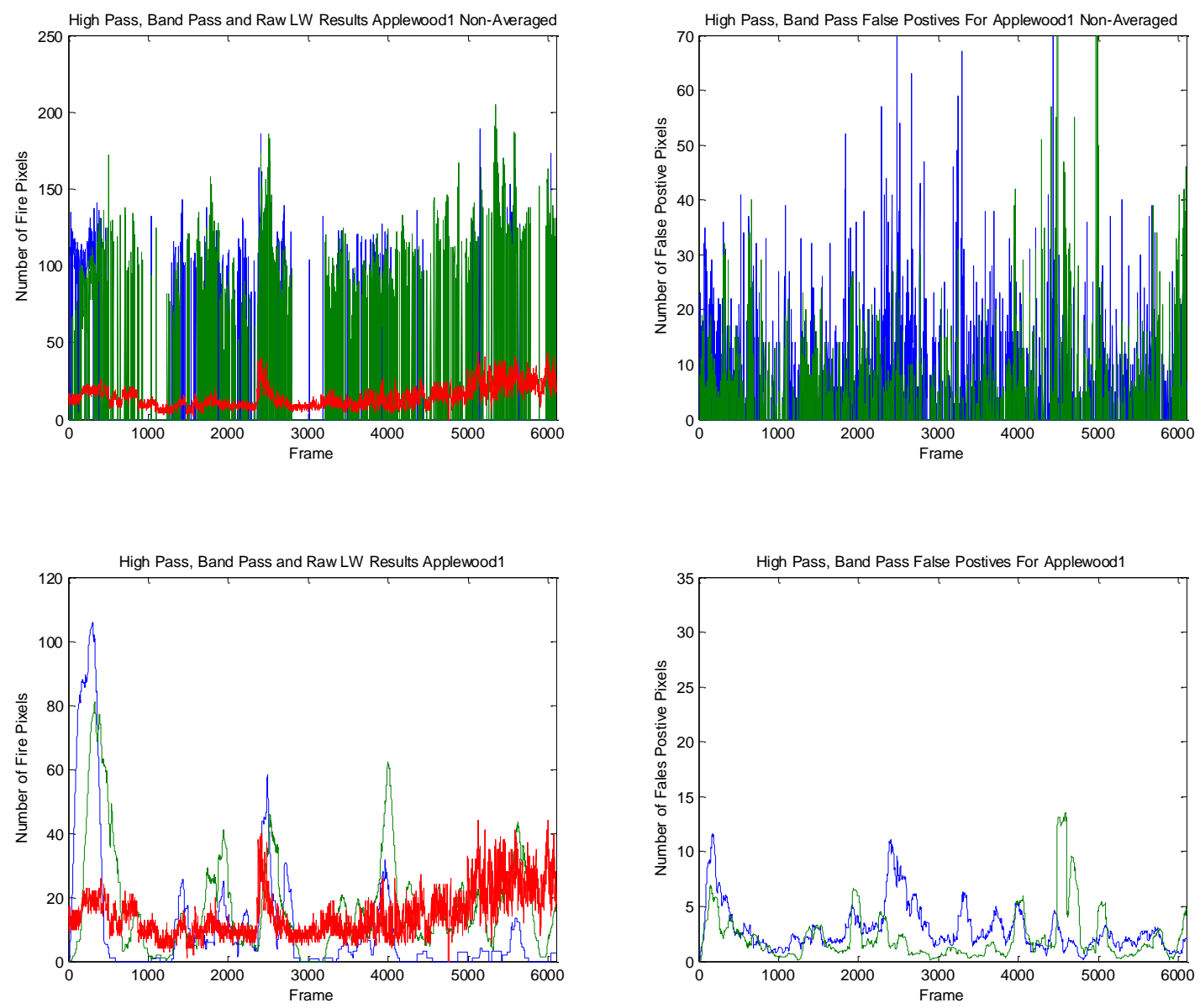

Page 77 


\section{Applewood2:}
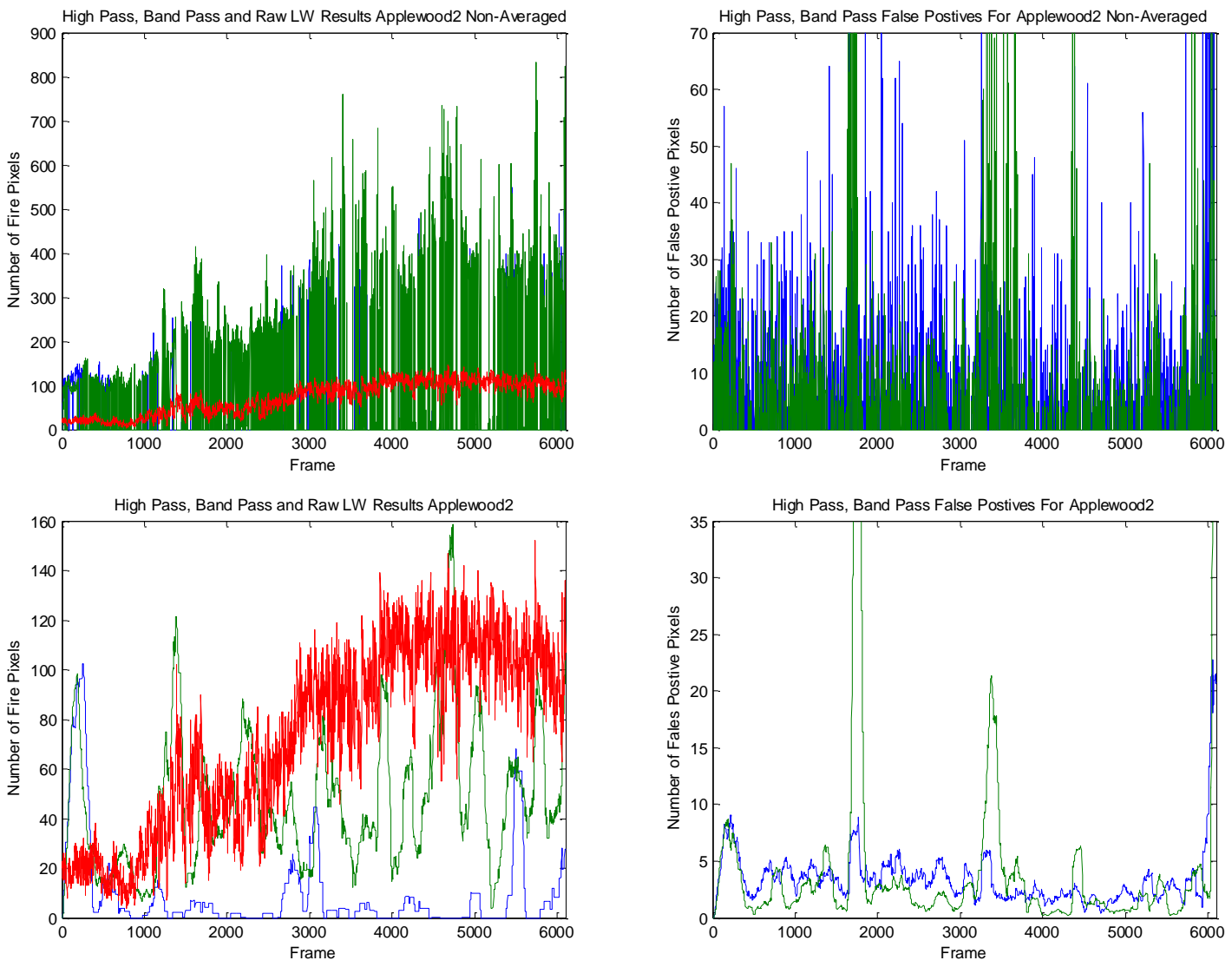

\section{Extinguish:}
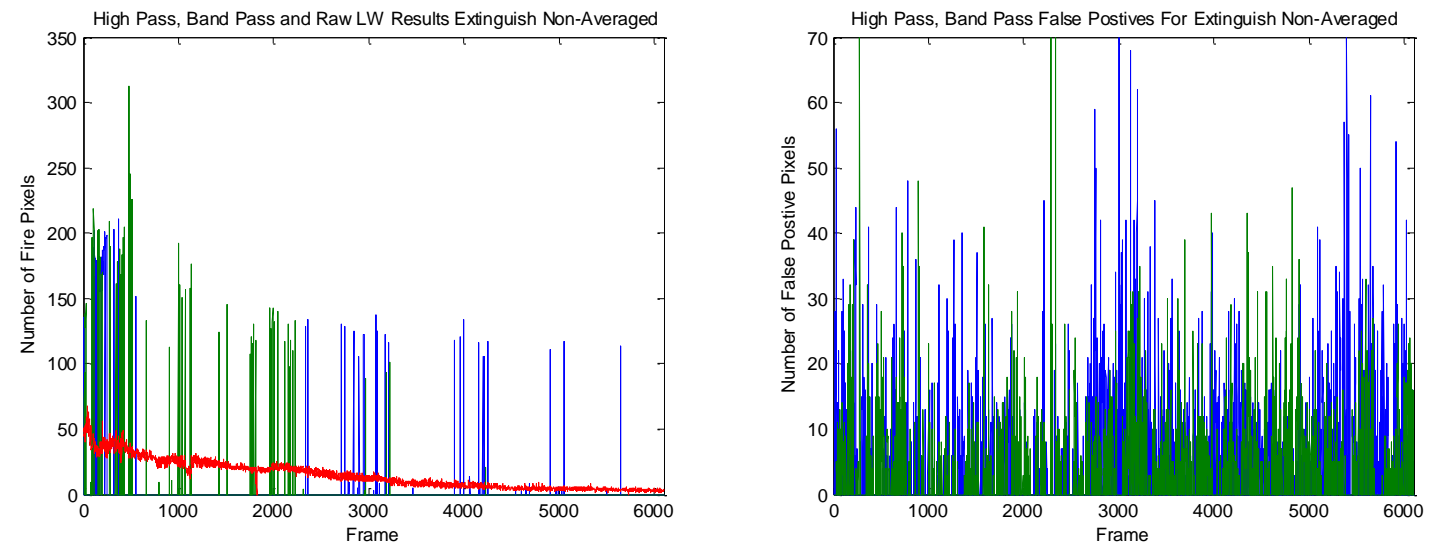

Page 78 

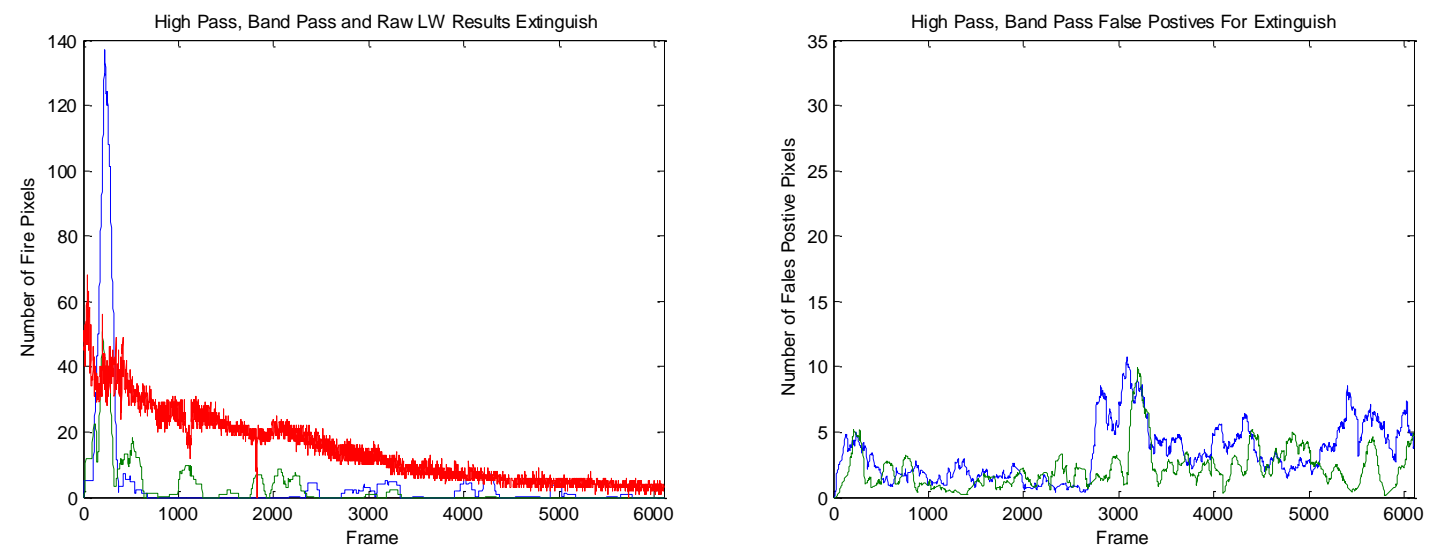

Page 79 


\section{APPENDIX B}

MATLAB Code Used

\begin{tabular}{|c|c|}
\hline File Name: & Brief Description \\
\hline b_generate_all.m & $\begin{array}{l}\text { Generates Filter Coefficients for both band } \\
\text { pass and high pass filter. It also generates } \\
\text { the selective gain image }\end{array}$ \\
\hline first_250_gen.m & $\begin{array}{l}\text { Generate the selective gain image from the } \\
\text { first } 250 \text { frames of charcoal data }\end{array}$ \\
\hline b_fir_highpass.m & Generates the high pass filter coefficients \\
\hline b_iir_bandpass.m & Generates the band pass filter coefficients \\
\hline b_fir_highpass_length.m & $\begin{array}{l}\text { Displays frequency response of high pass } \\
\text { filter with different filter lengths }\end{array}$ \\
\hline b_iir_bandpass_length.m & $\begin{array}{l}\text { Displays frequency response of band pass } \\
\text { filter with different filter lengths }\end{array}$ \\
\hline small_pix_gen.m & $\begin{array}{l}\text { Generates } 128 \text { by } 128 \text { by } 8000 \text { matrix with } \\
\text { oats } 1 \text { frames centered on the fire location }\end{array}$ \\
\hline edge_freq_data.m & Generates all the signal type plots \\
\hline b_fir_filter_test_dual.m & Runs the high pass filter on a set of data \\
\hline b_iir_filter_test_dual.m & Runs the band pass filter on a set of data \\
\hline b_batch_movie.m & $\begin{array}{l}\text { Generates movies and results for all data } \\
\text { sets }\end{array}$ \\
\hline b_results_plots.m & Displays results for all data sets \\
\hline \multicolumn{2}{|l|}{ b_generate_all.m } \\
\hline \multicolumn{2}{|c|}{$\begin{array}{l}\text { \% this file generates all the variables required for the rest of the } \\
\text { files } \\
\text { o including filter coefficients and selective gain matrix } \\
\text { addpath('c: \matlab files\dsp'); } \\
\text { o create baseline files } \\
\text { first } 250 \text { gen }\end{array}$} \\
\hline
\end{tabular}




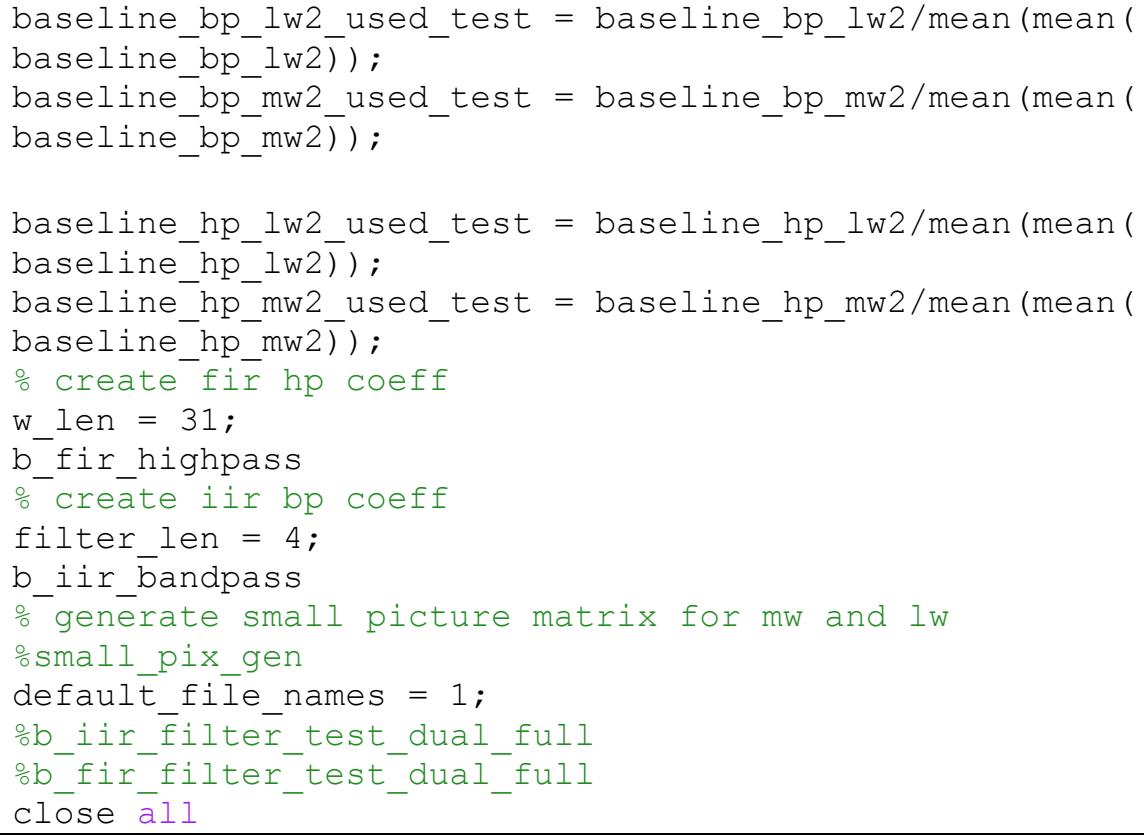

\section{first_250_gen.m}

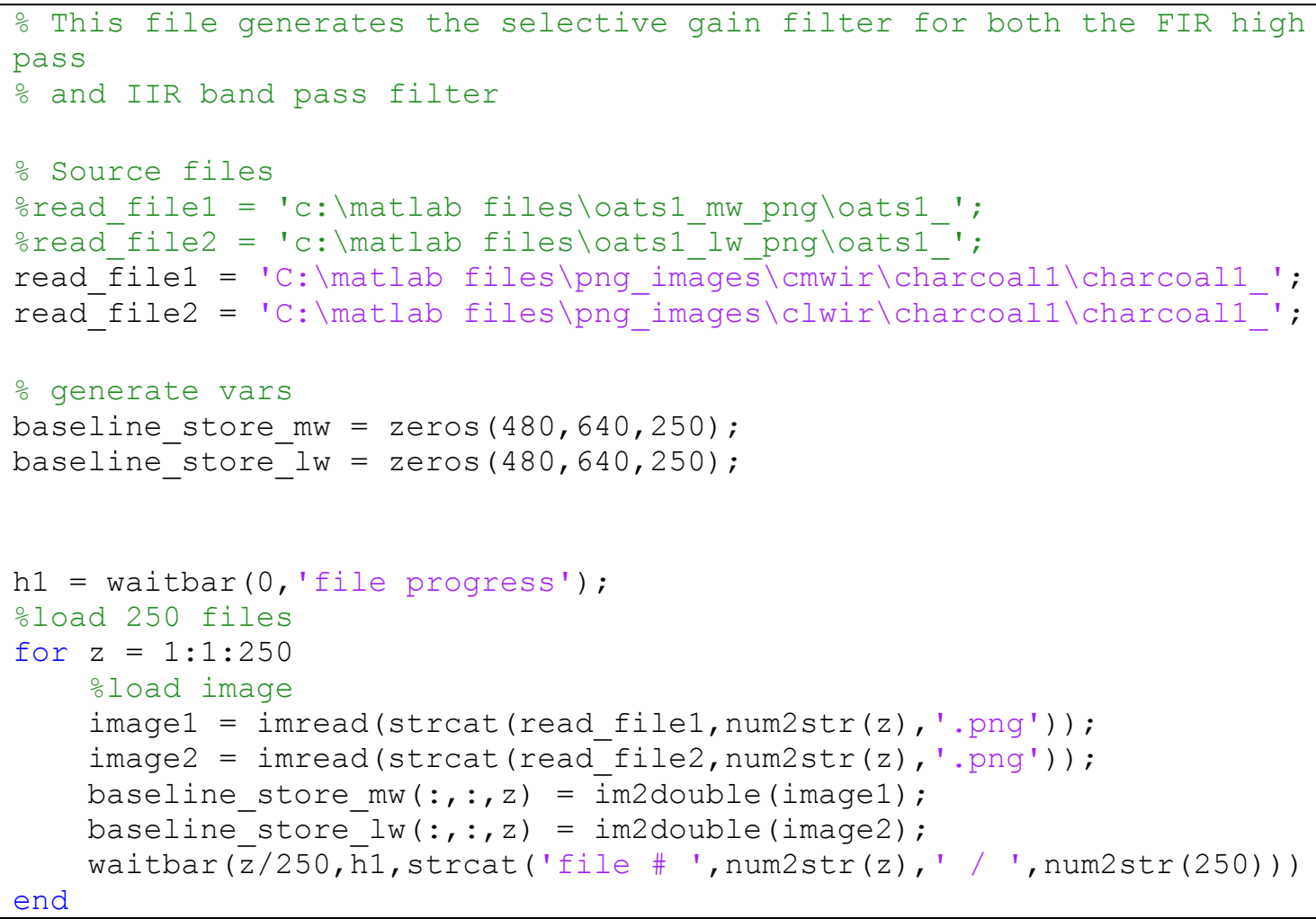

\section{Page 81}




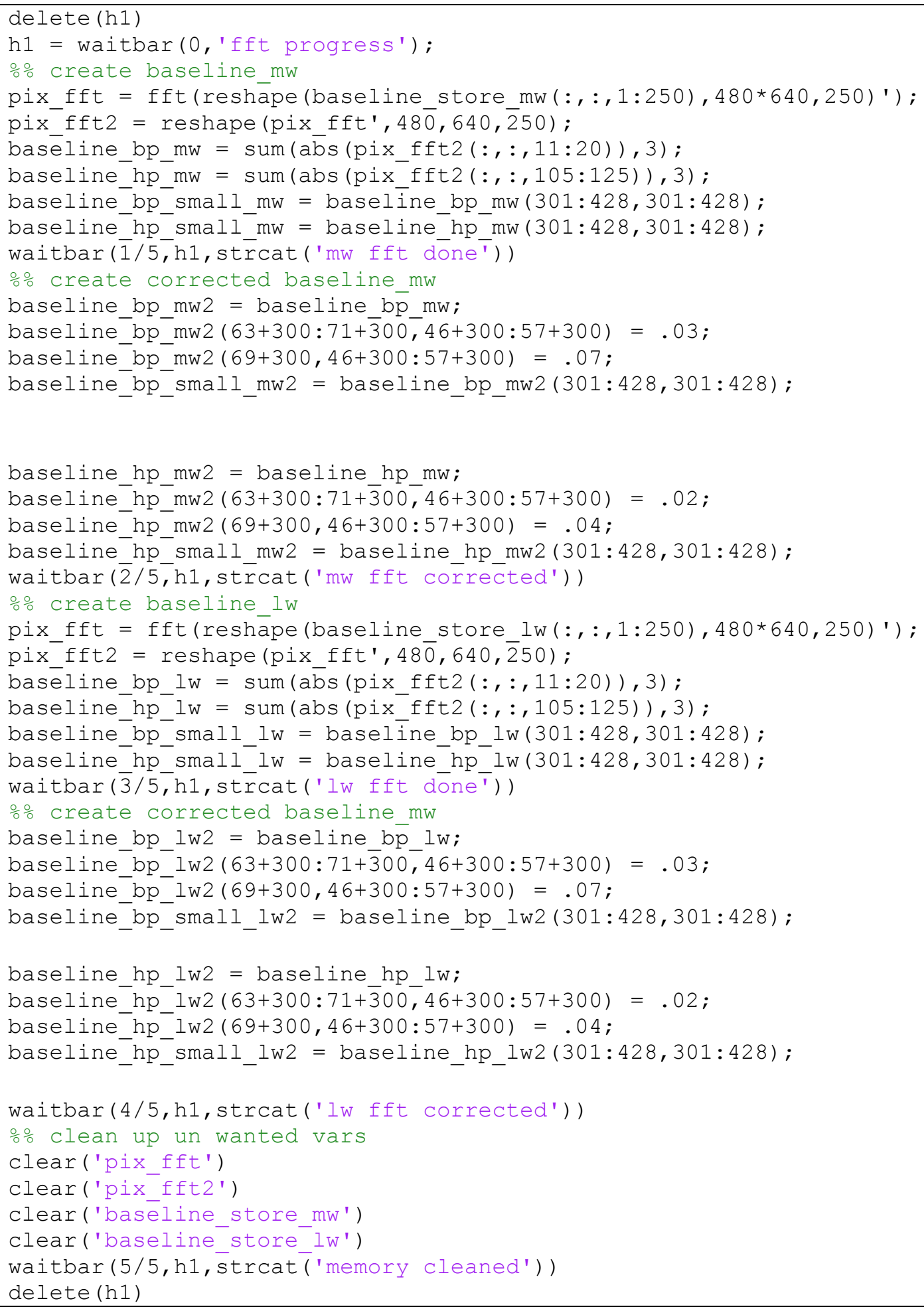

\section{b_fir_highpass.m}

\section{Page 82}




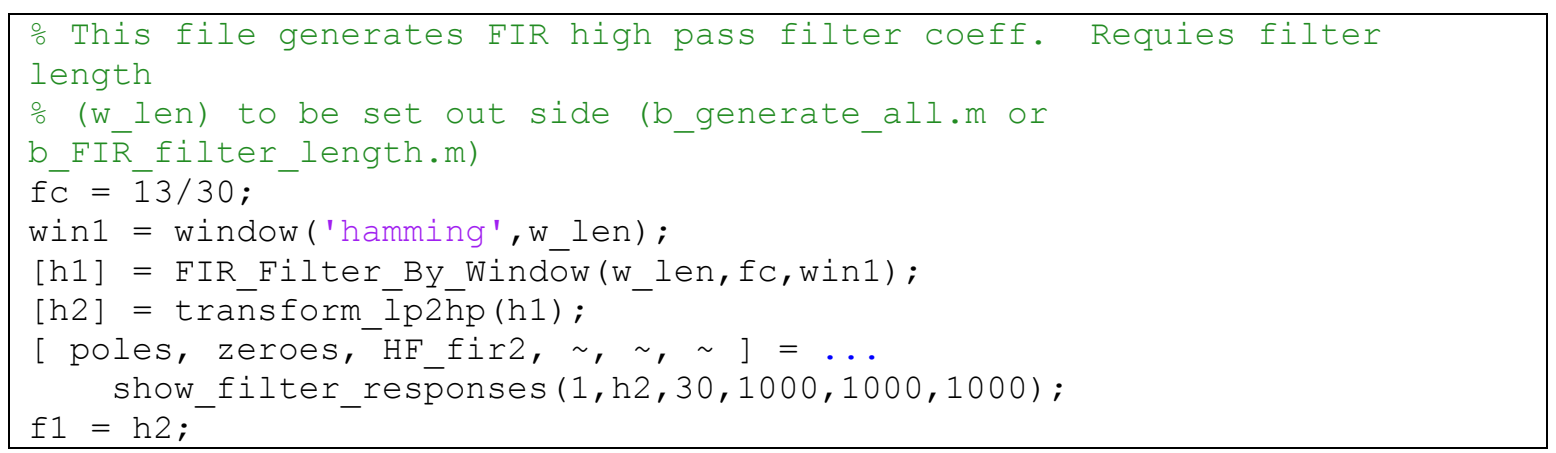

\section{b_iir_bandpass.m}

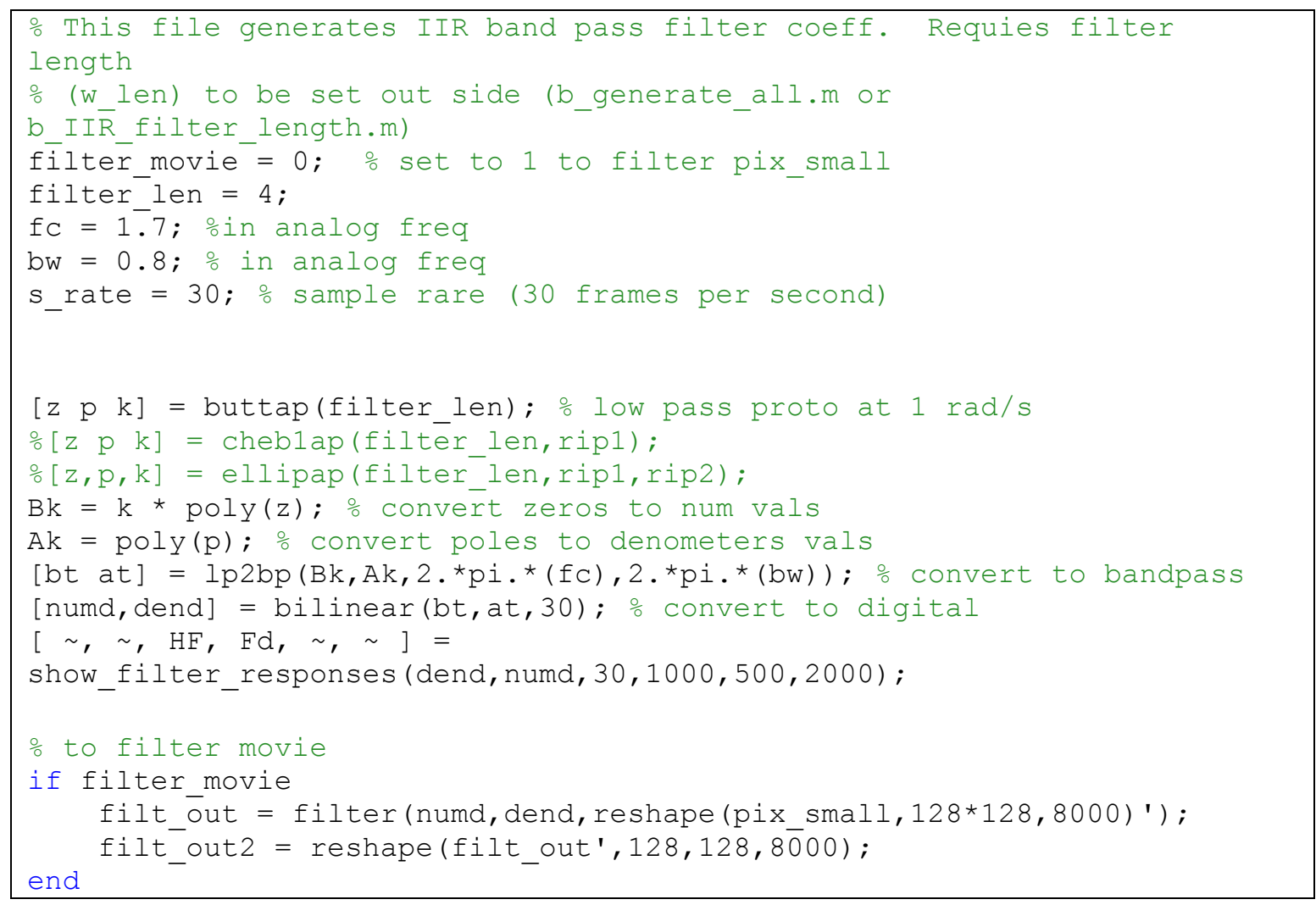

\section{b_fir_highpass_length.m}

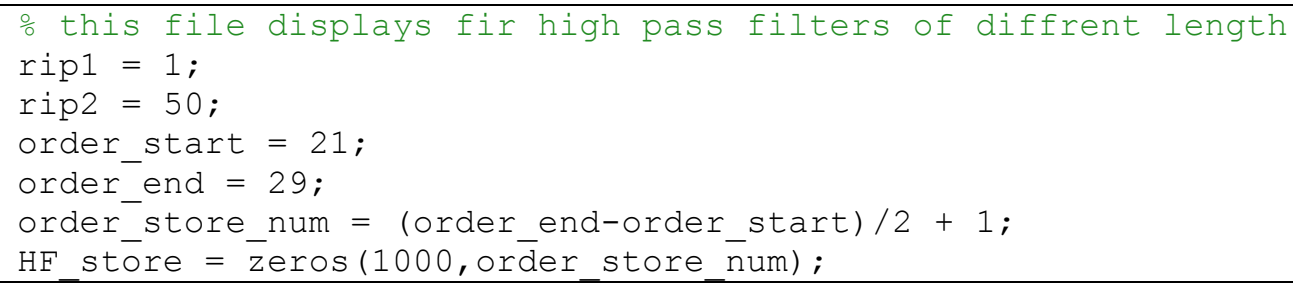

\section{Page 83}




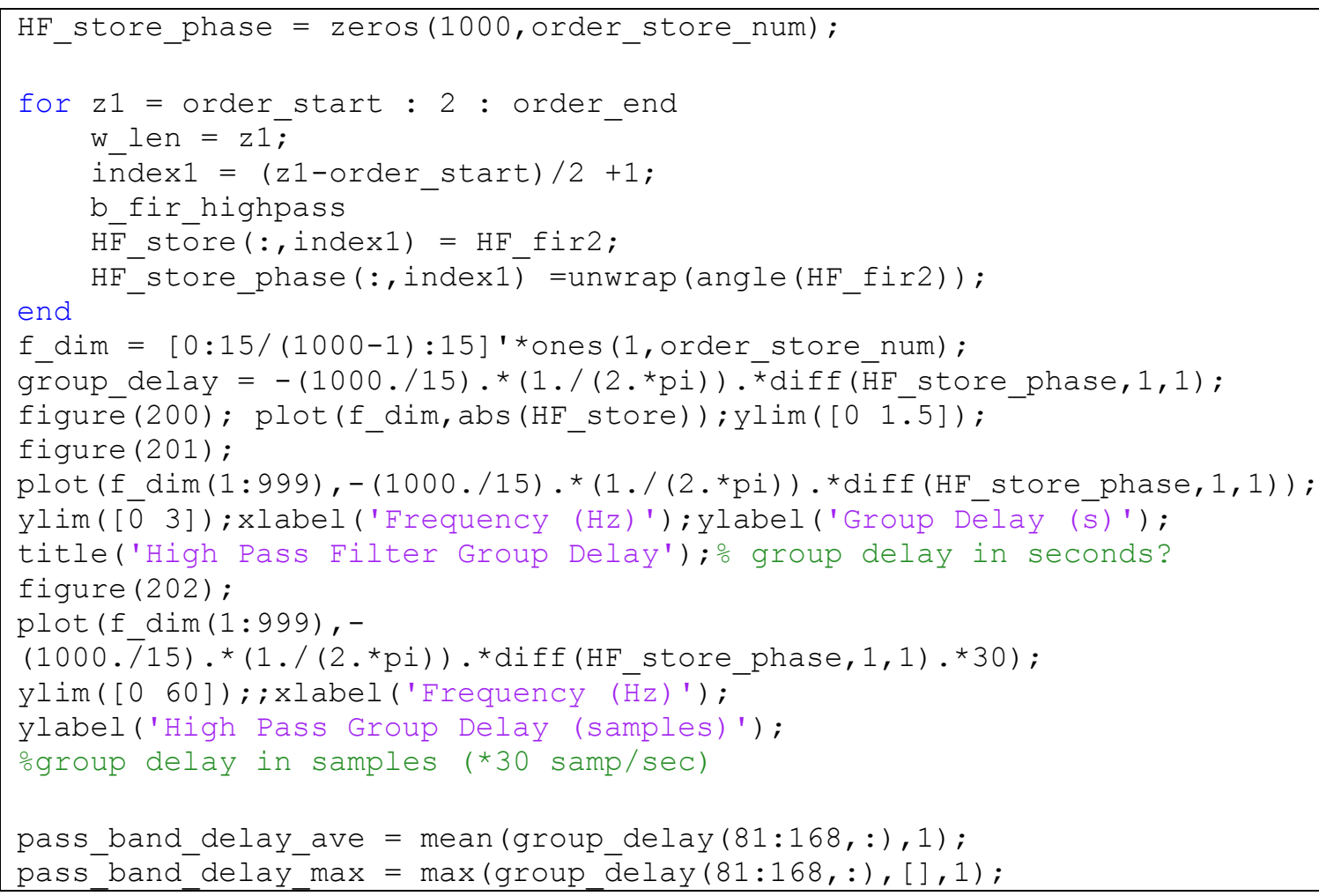

\section{b_iir_bandpass_length.m}

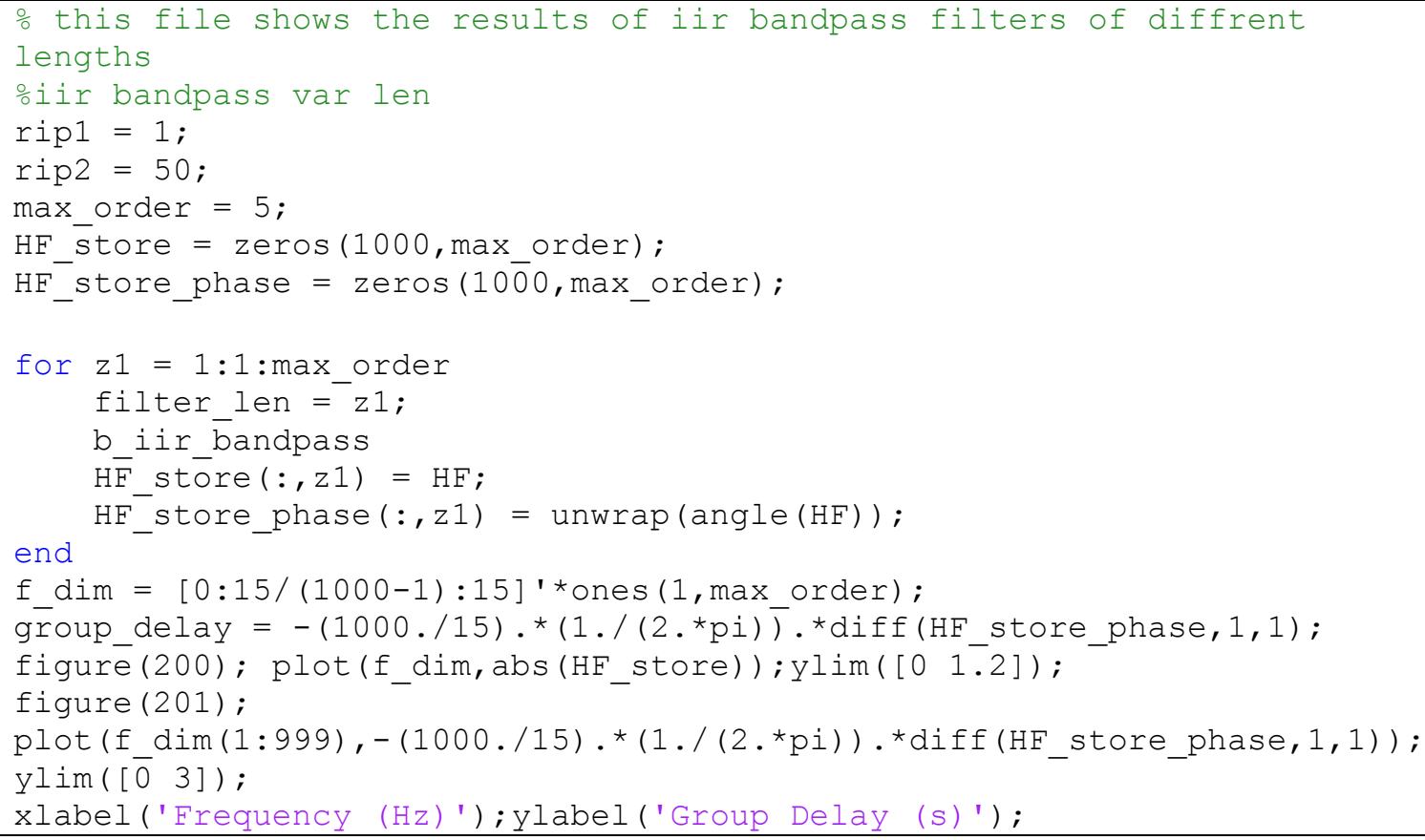

\section{Page 84}




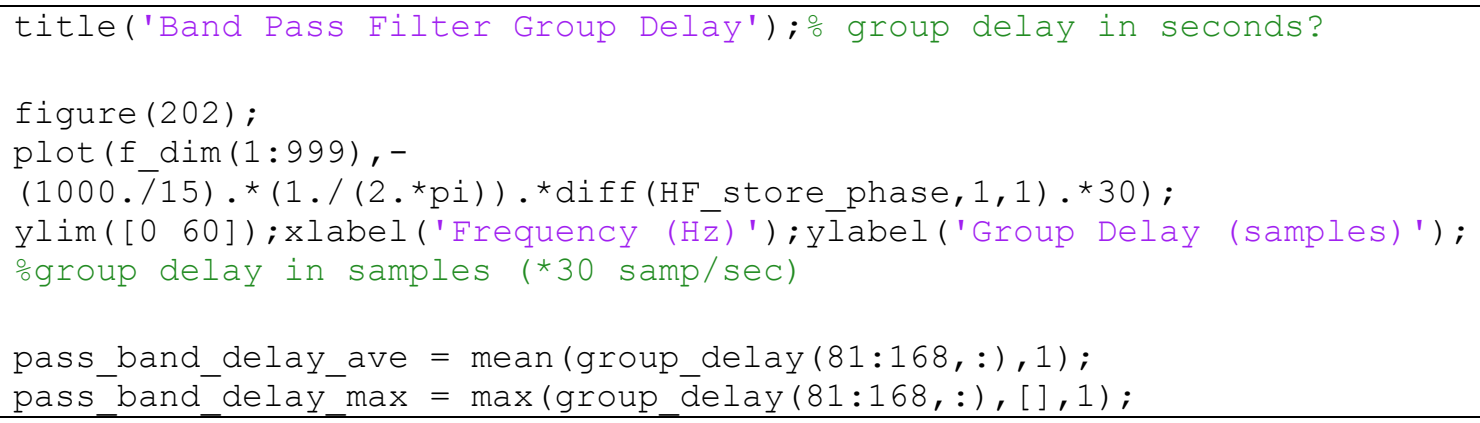

\section{small_pix_gen.m:}

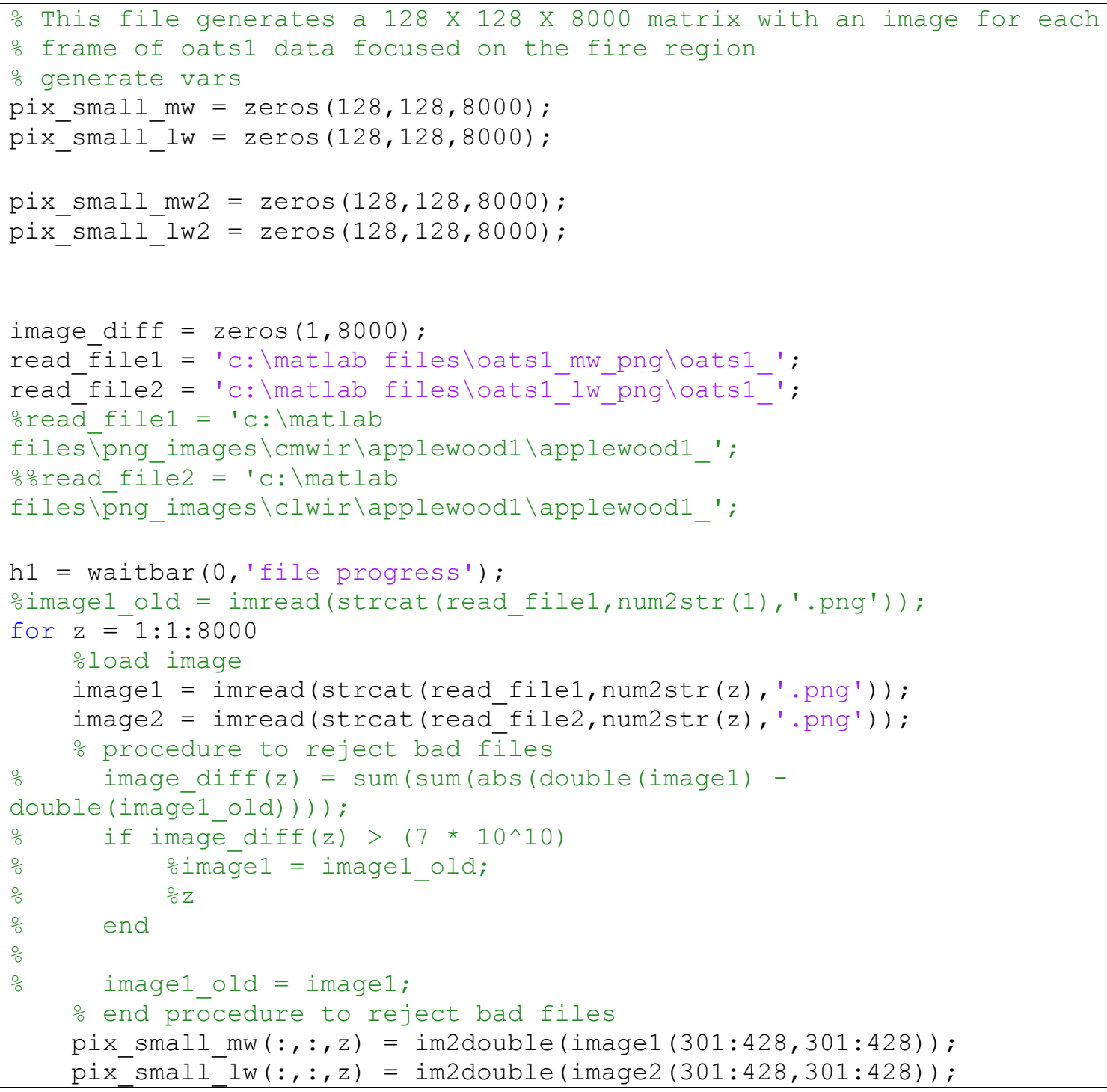

\section{Page 85}




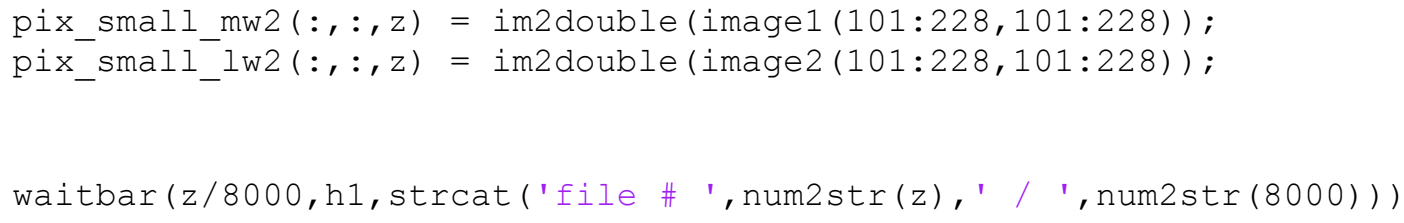

\section{edge_freq_data.m}

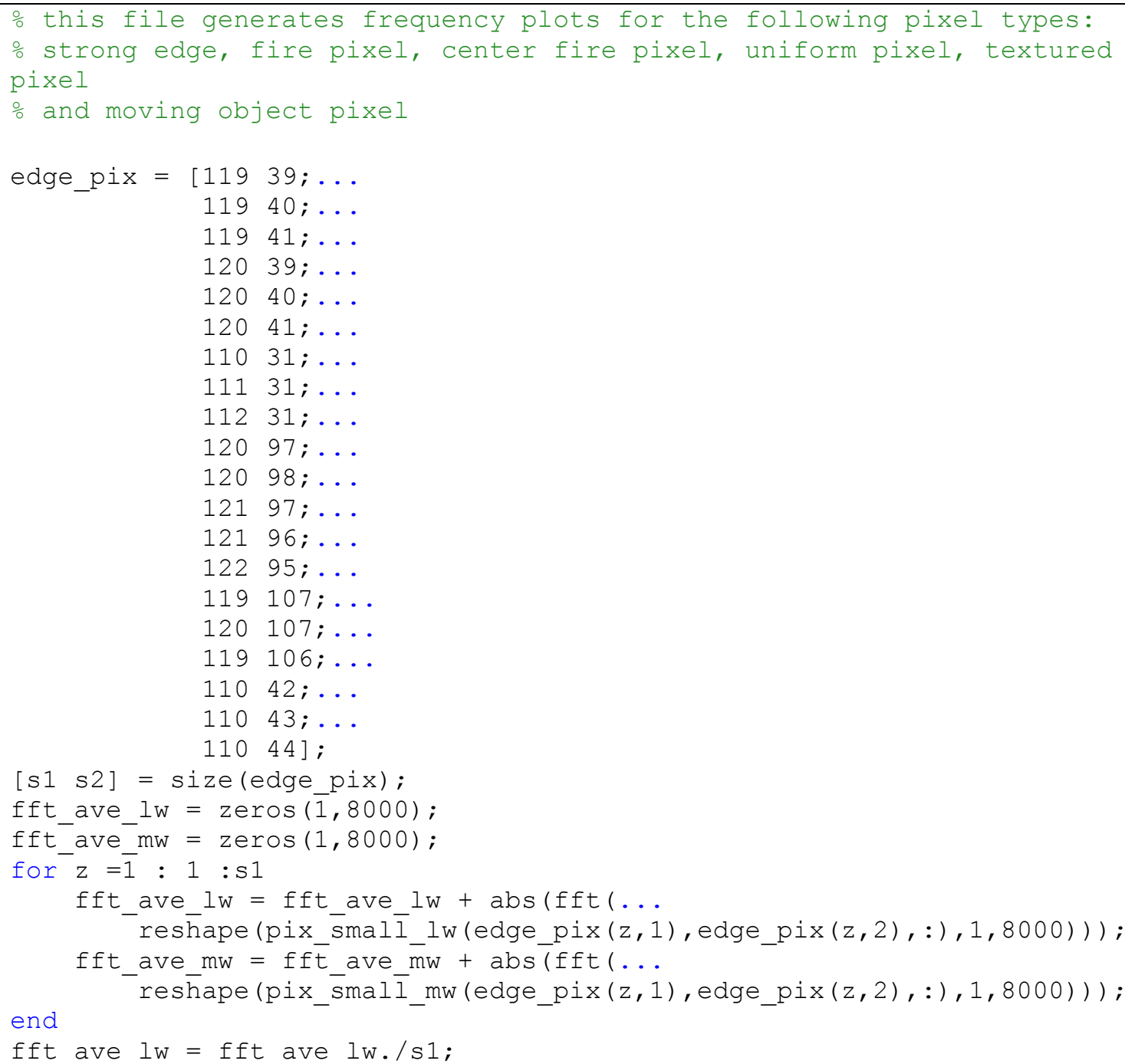

\section{Page 86}




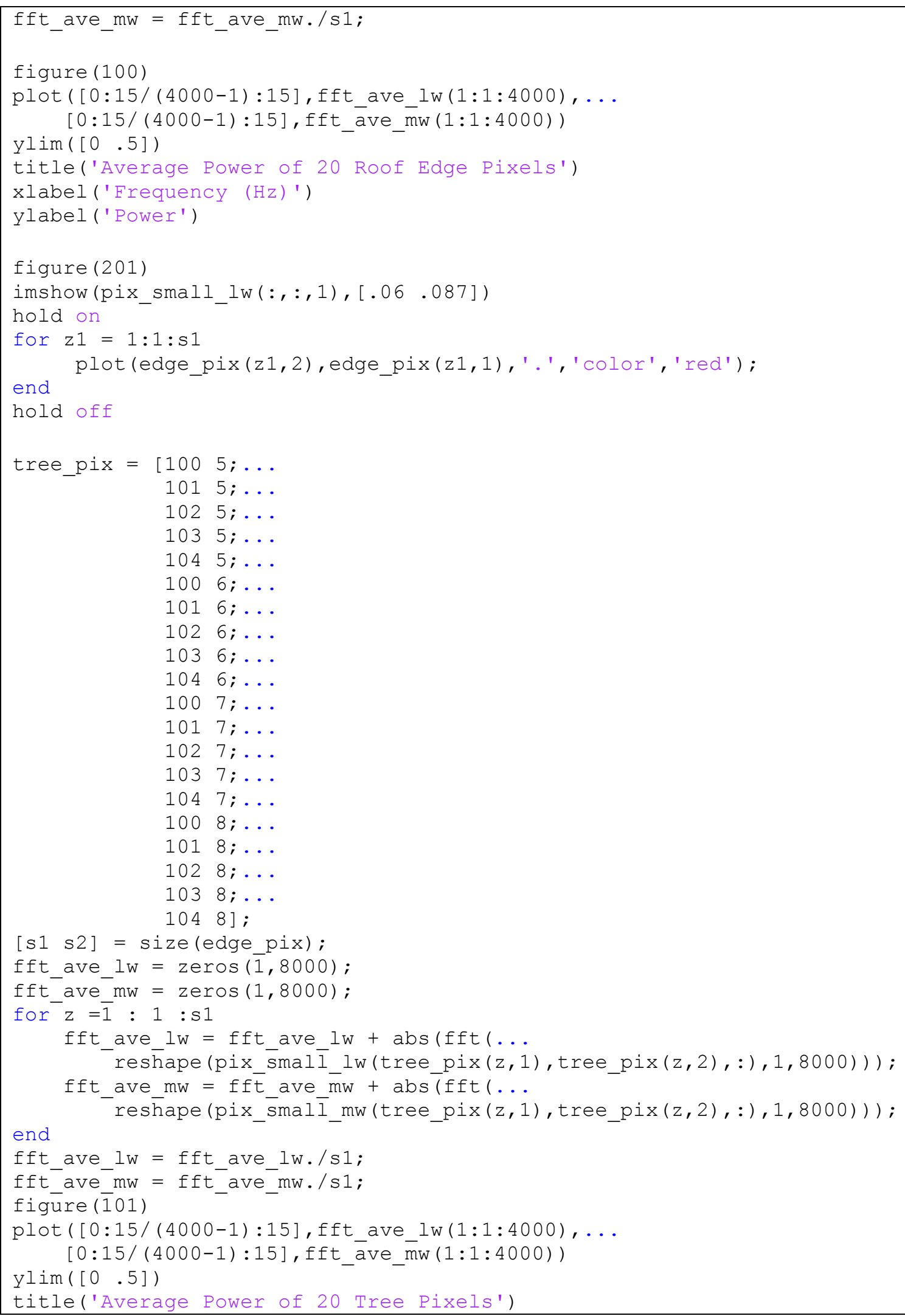

\section{Page 87}




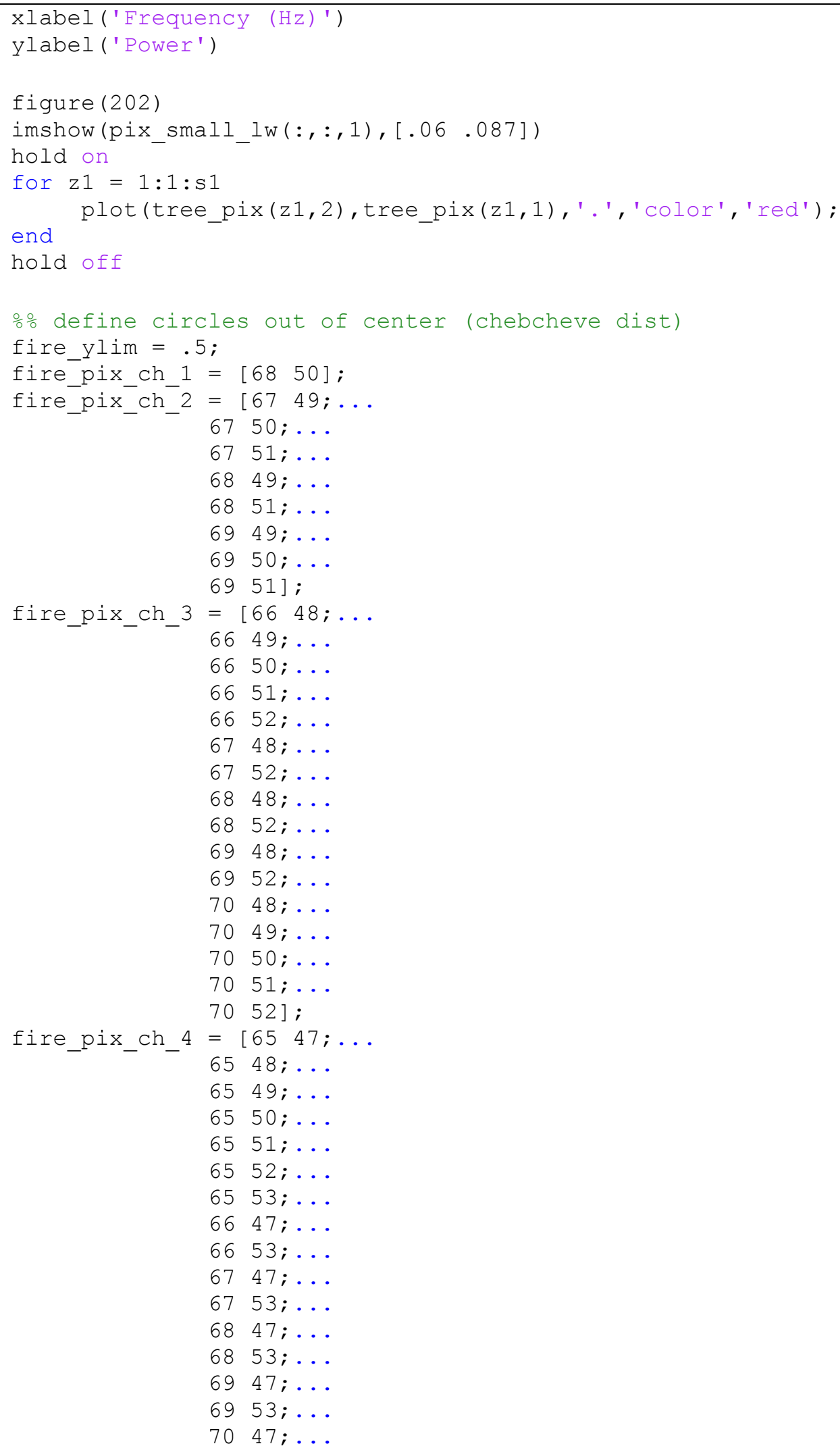

\section{Page 88}




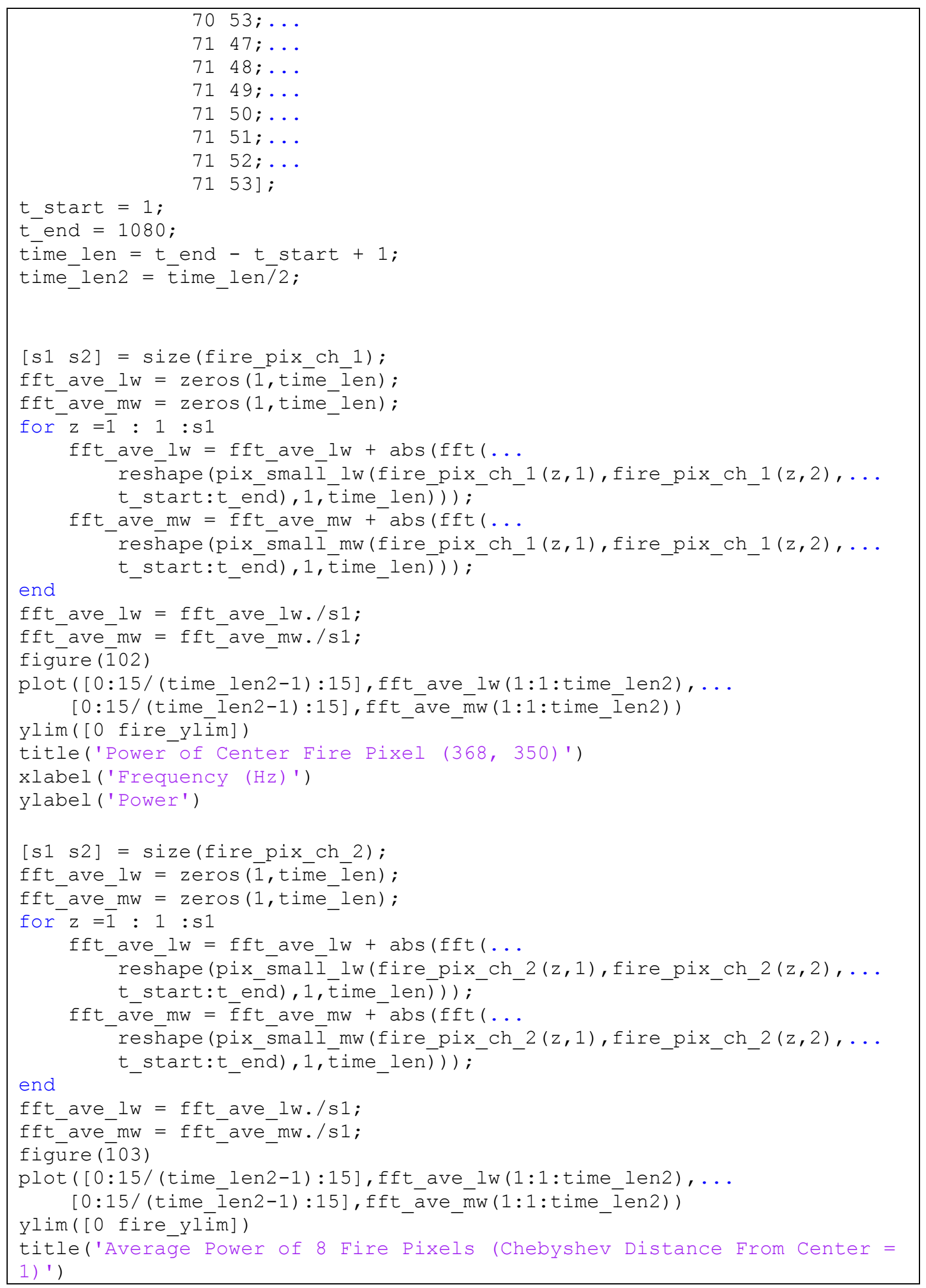

\section{Page 89}




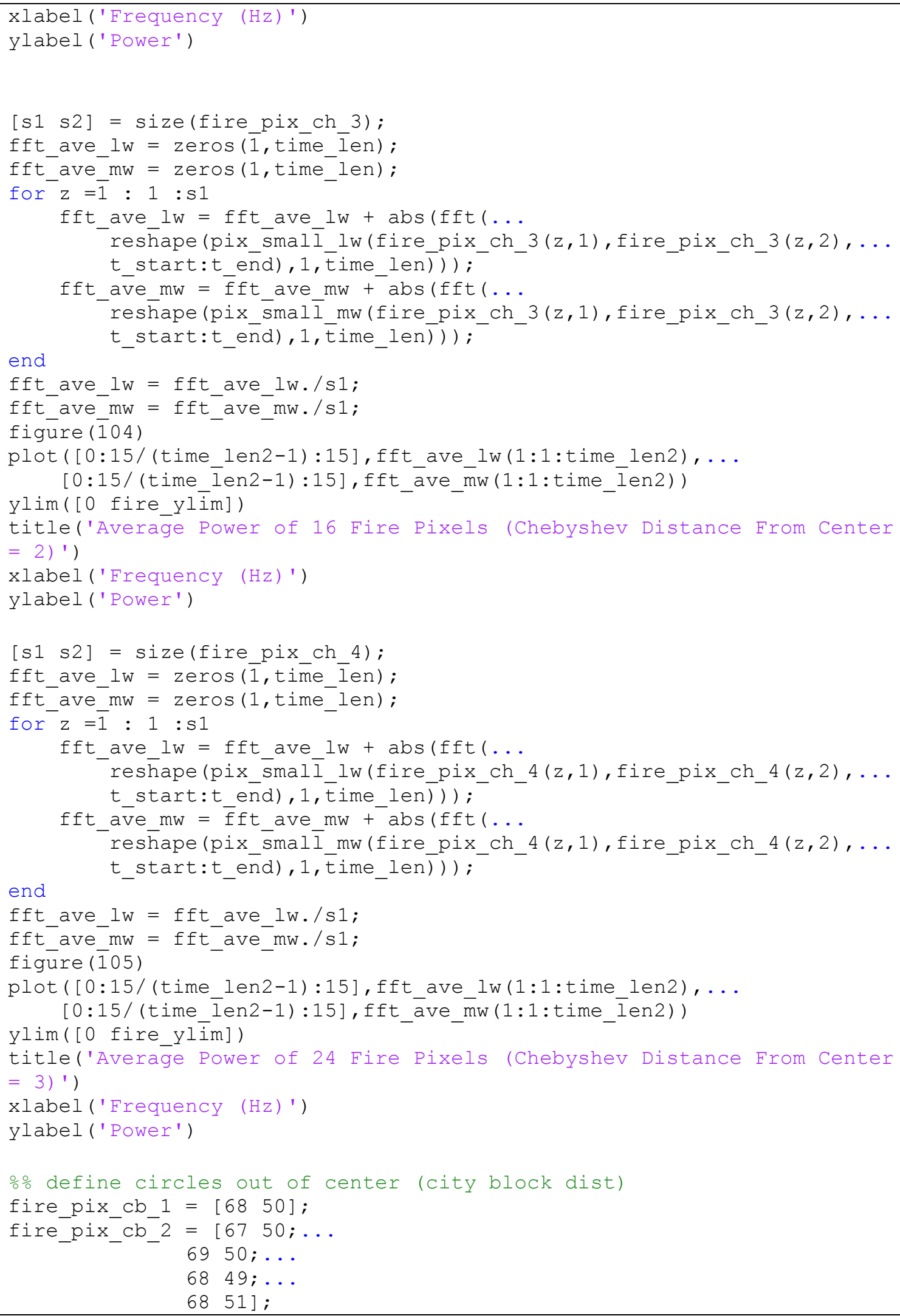




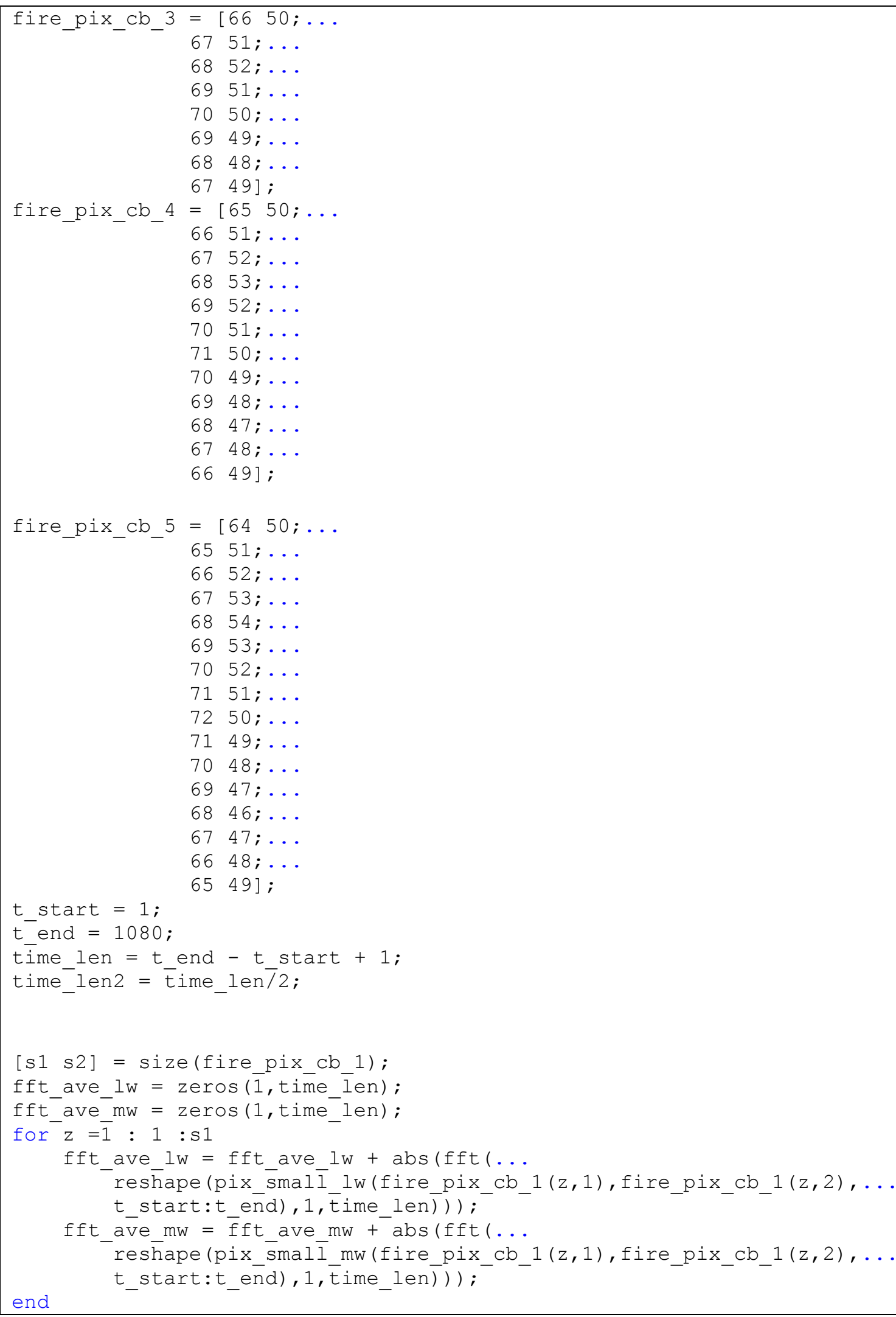

\section{Page 91}




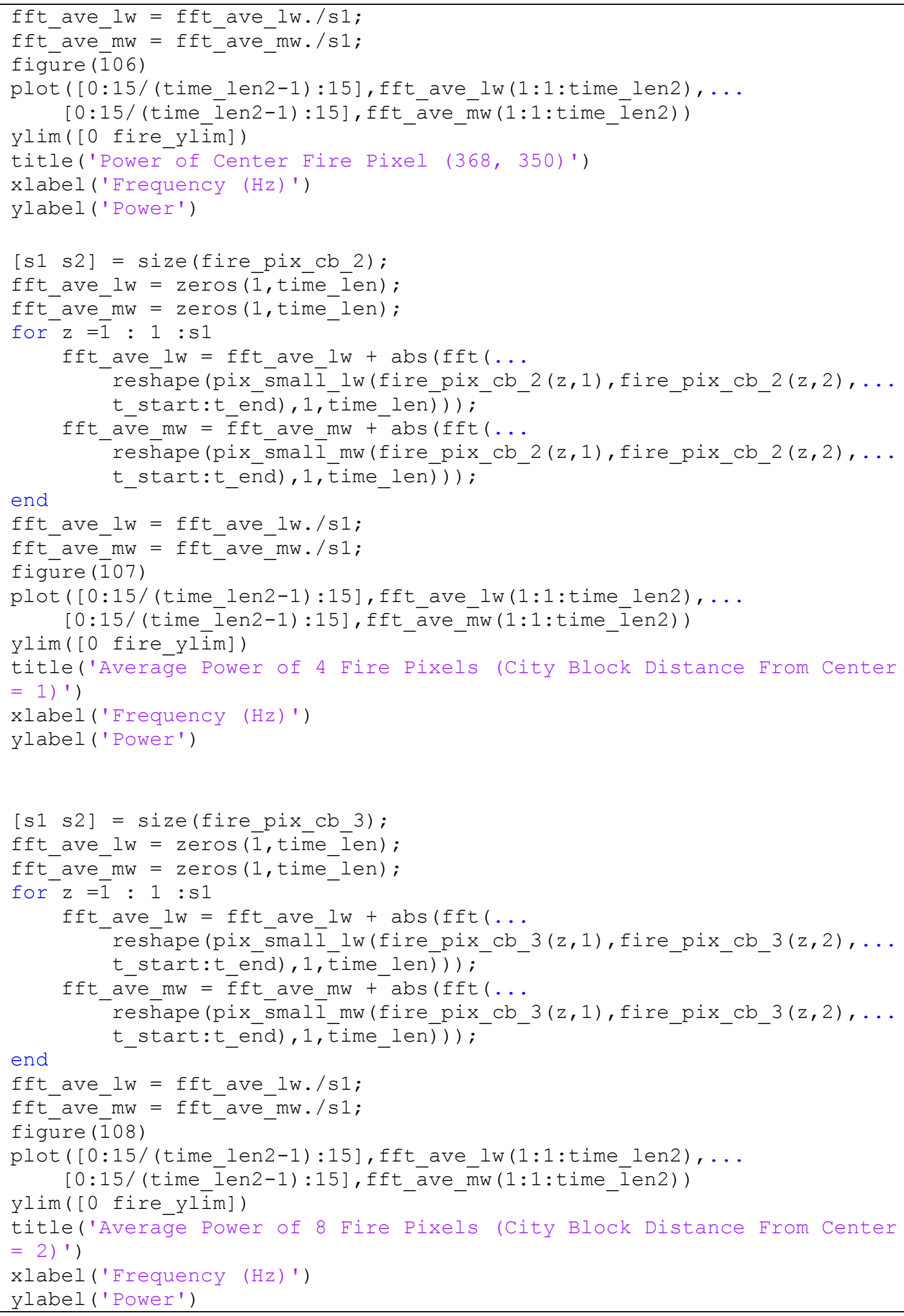




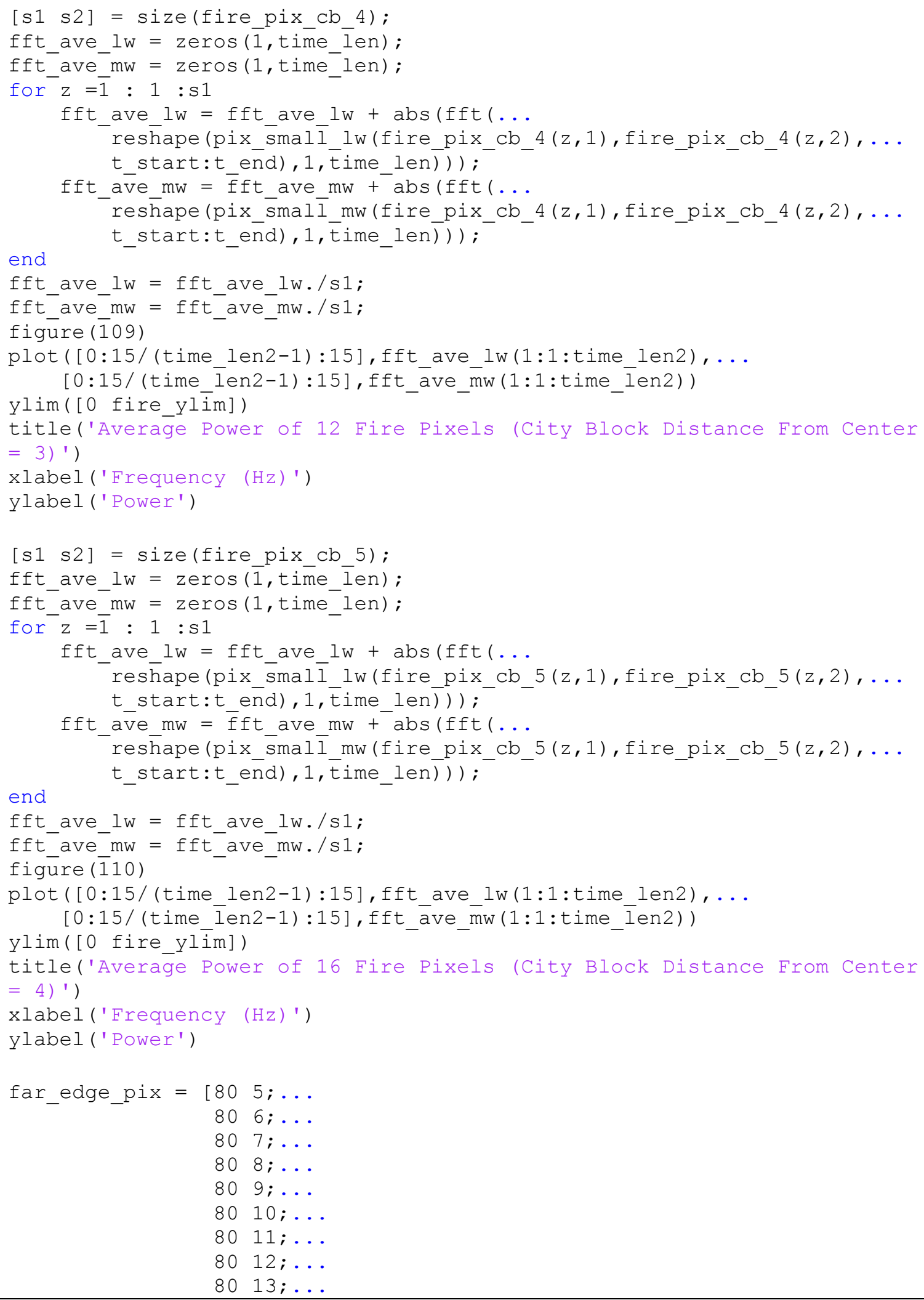

\section{Page 93}




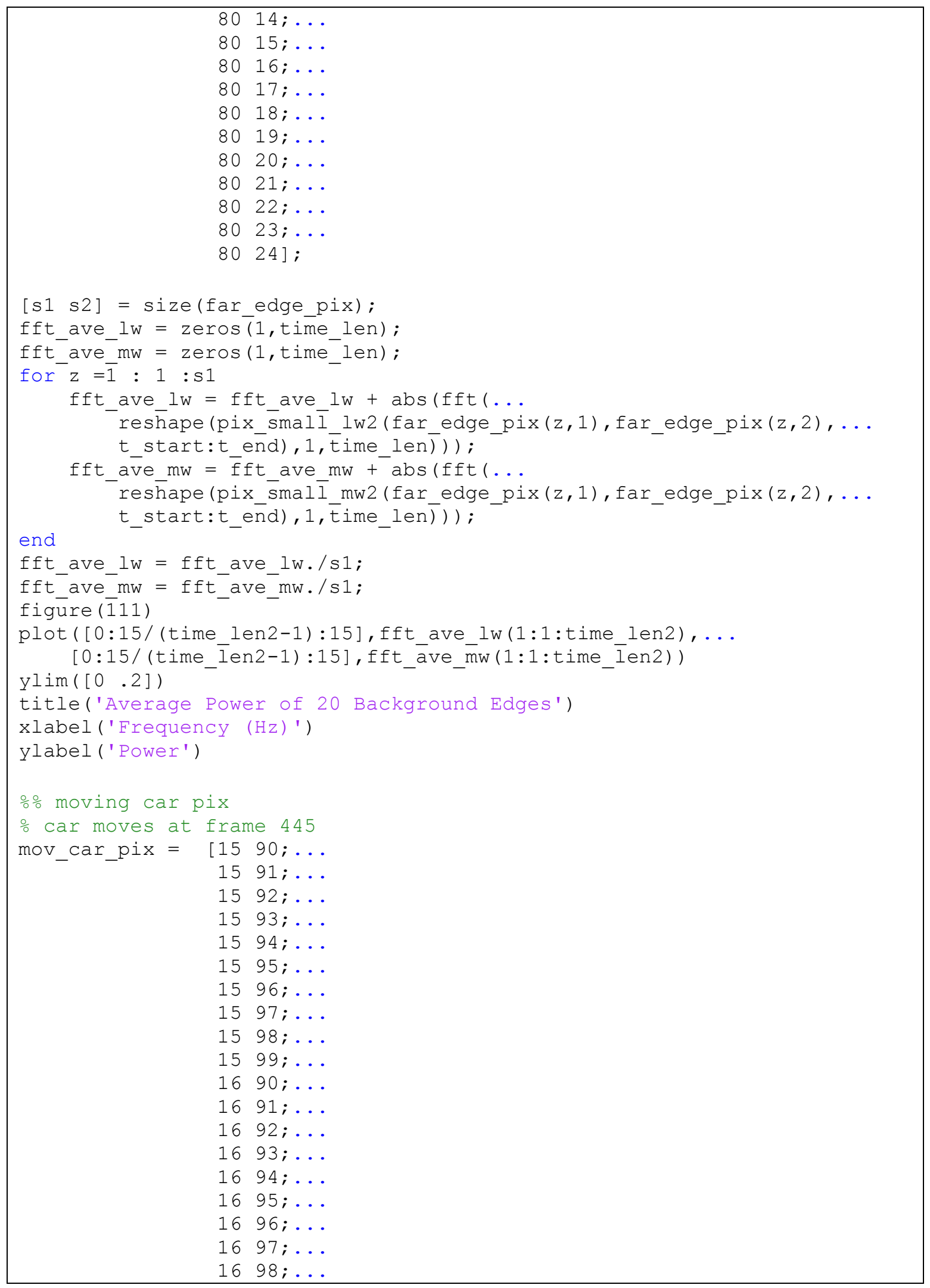

Page 94 


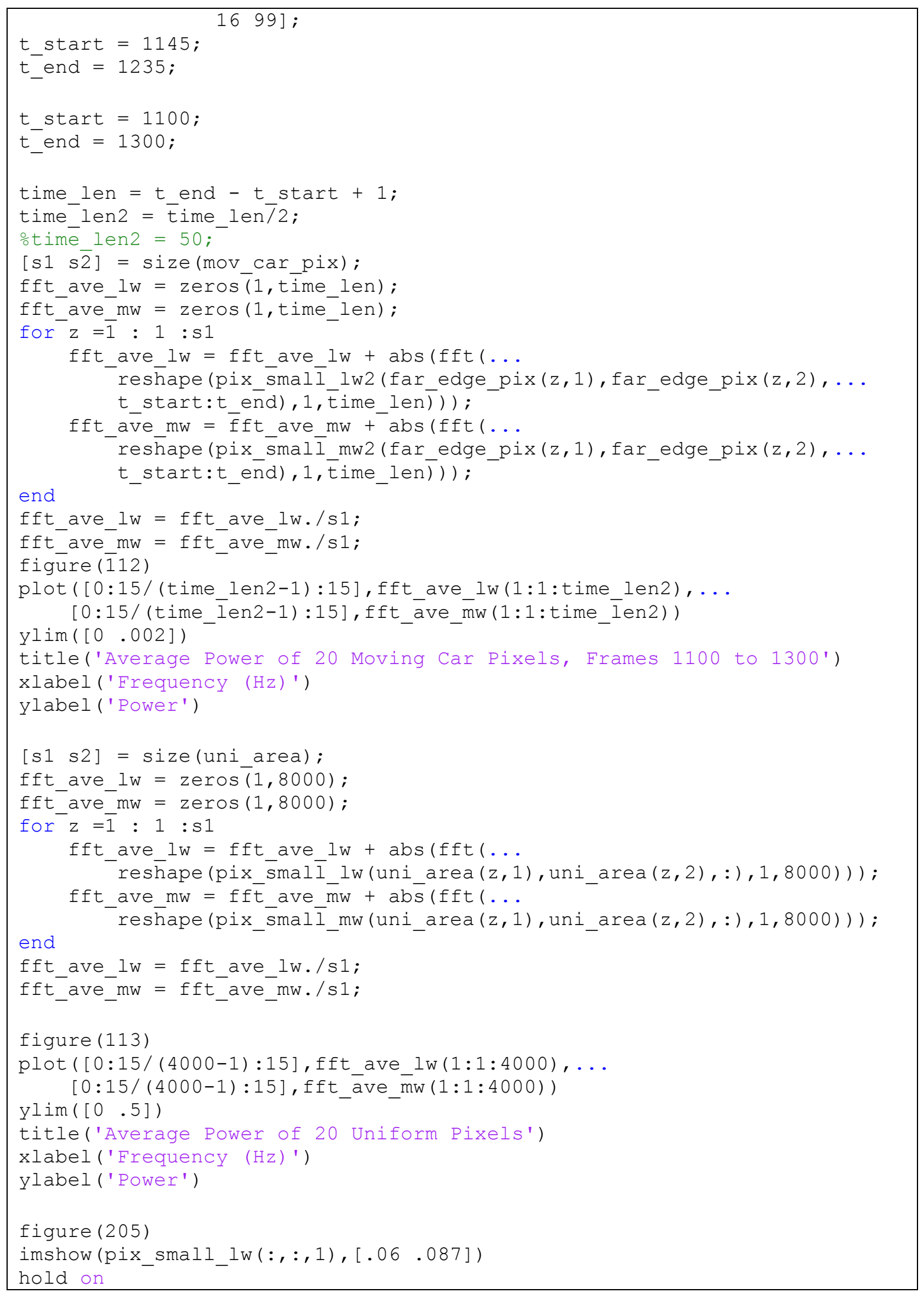

\section{Page 95}




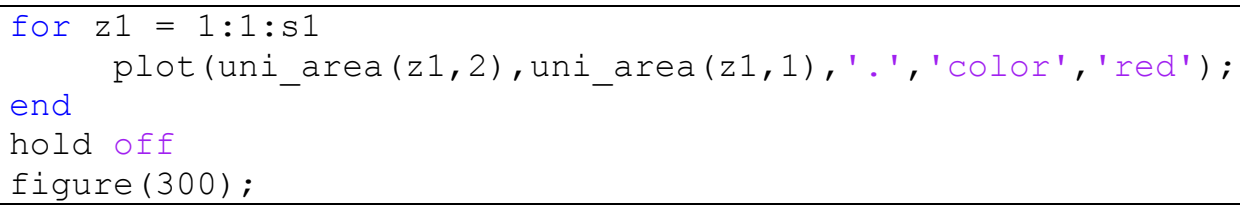

\section{b_fir_filter_test_dual.m:}

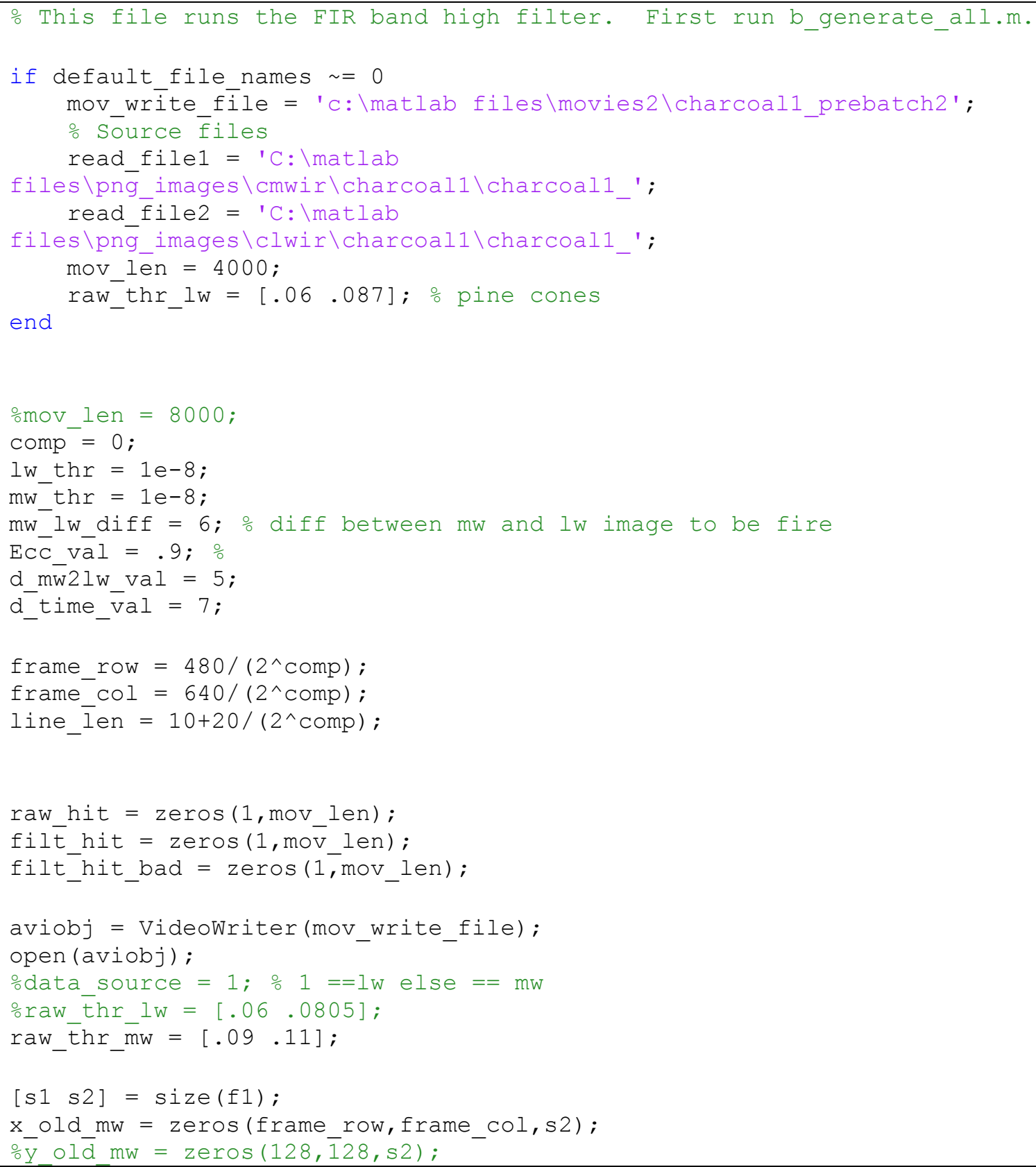




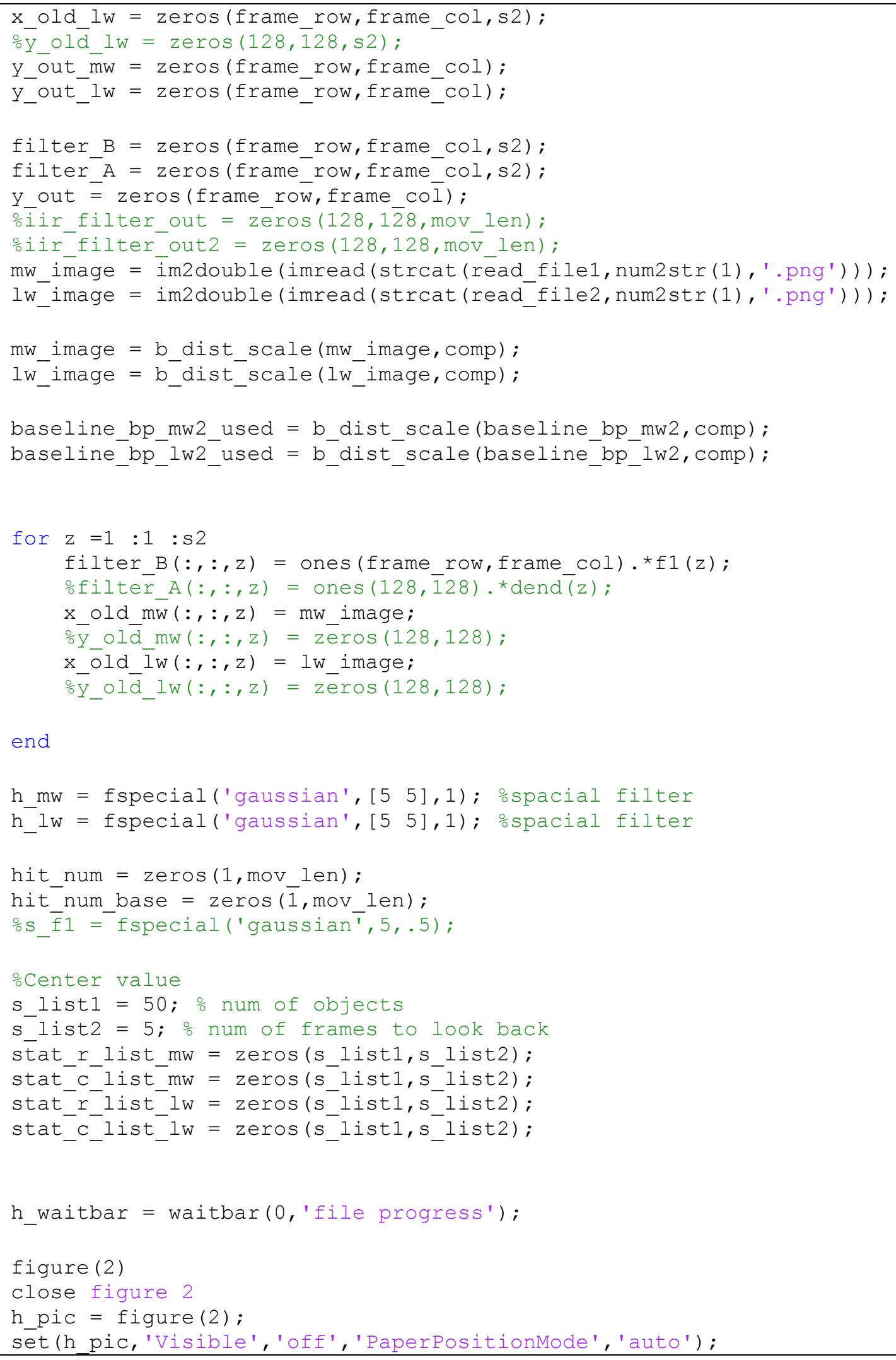

\section{Page 97}


figure (1)

close figure 1

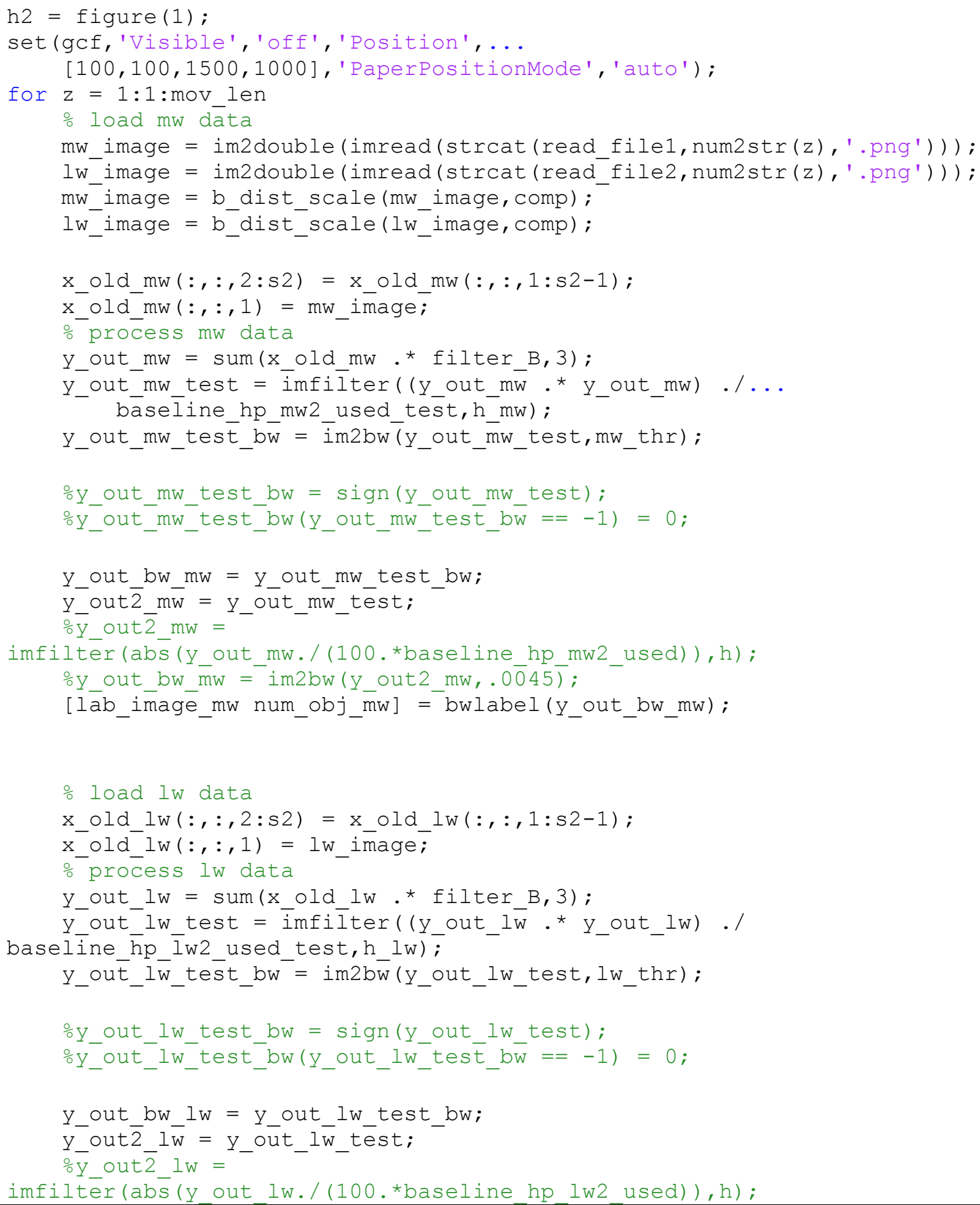

\section{Page 98}




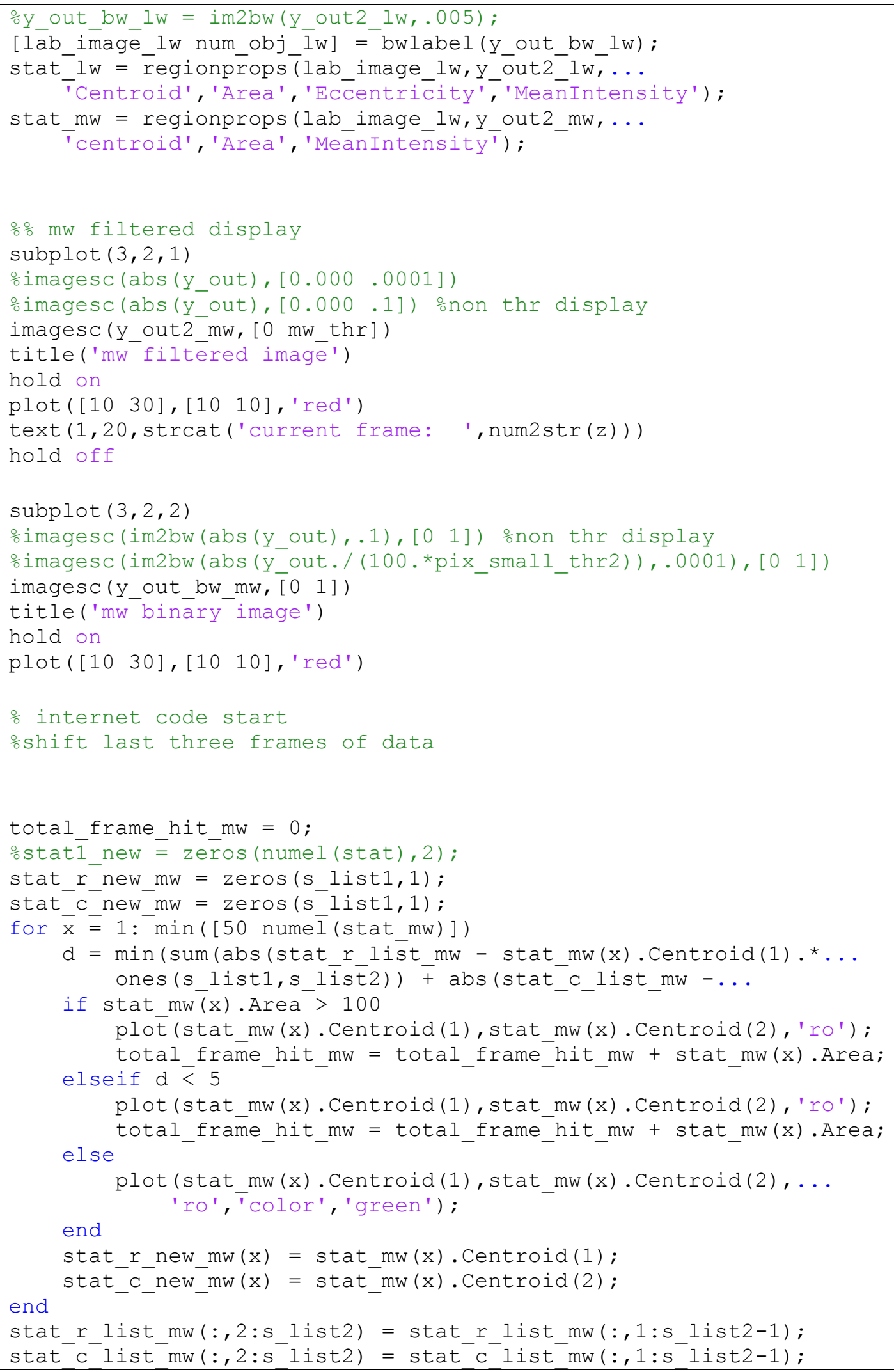

\section{Page 99}




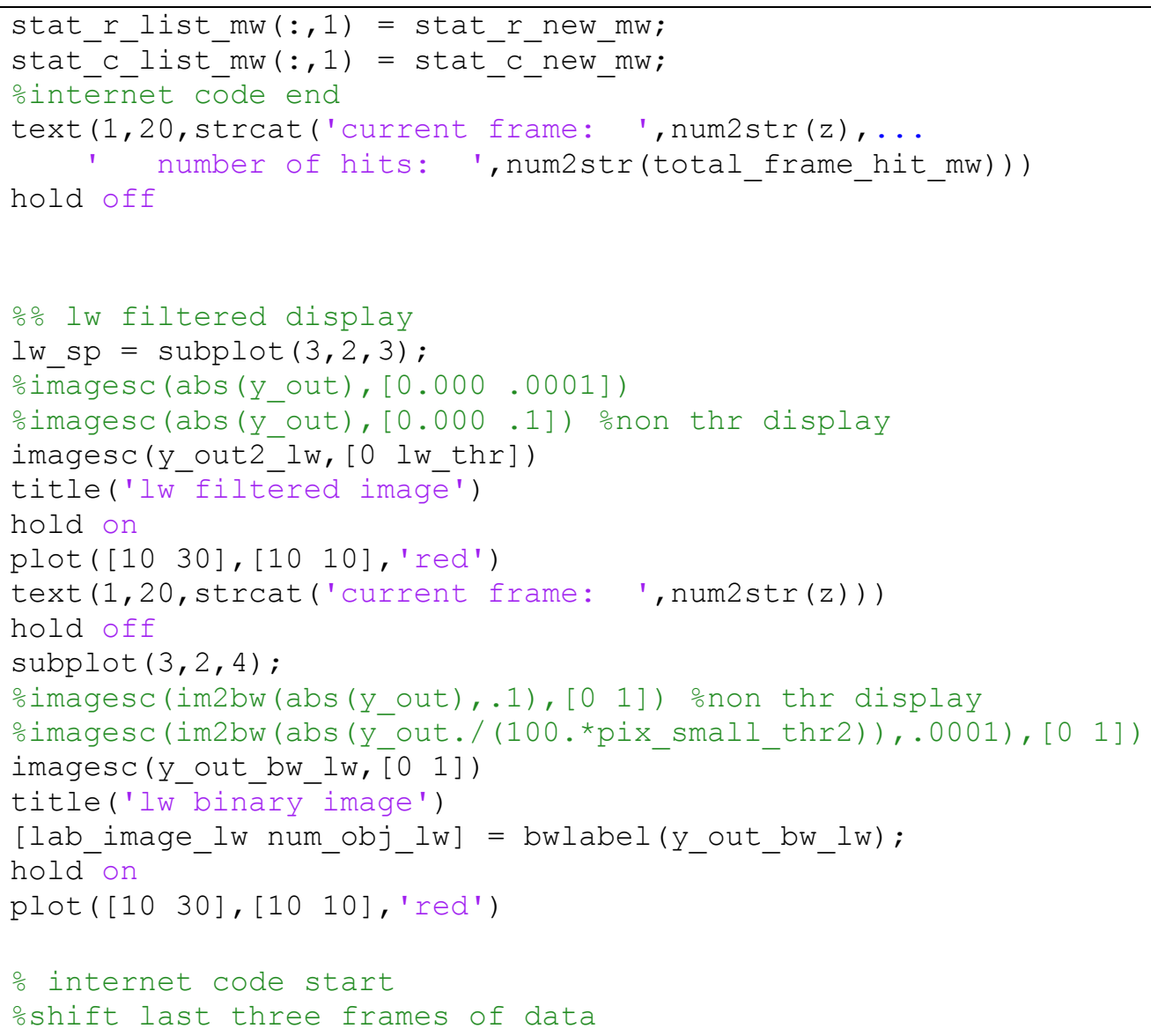

\section{Page 100}




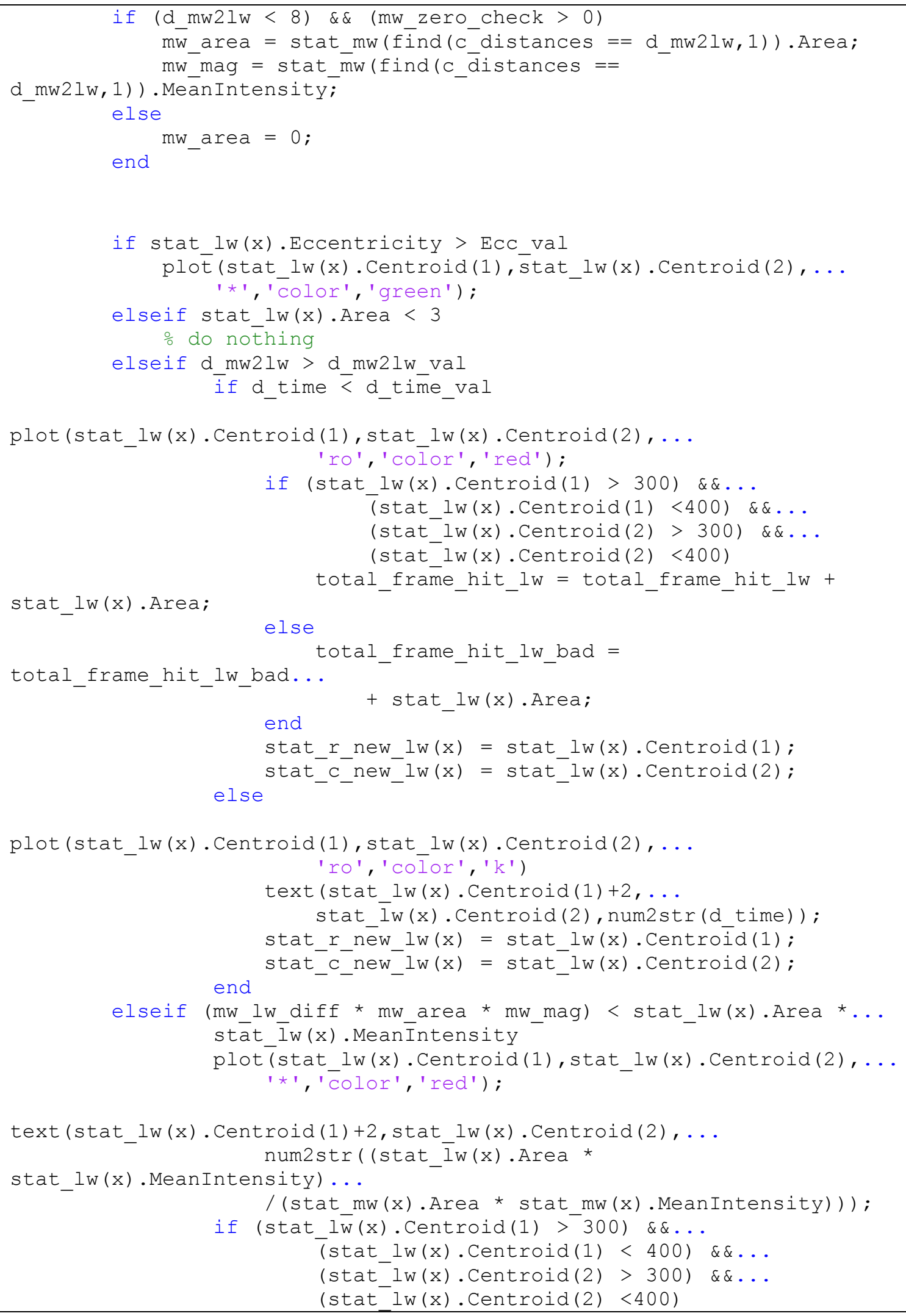




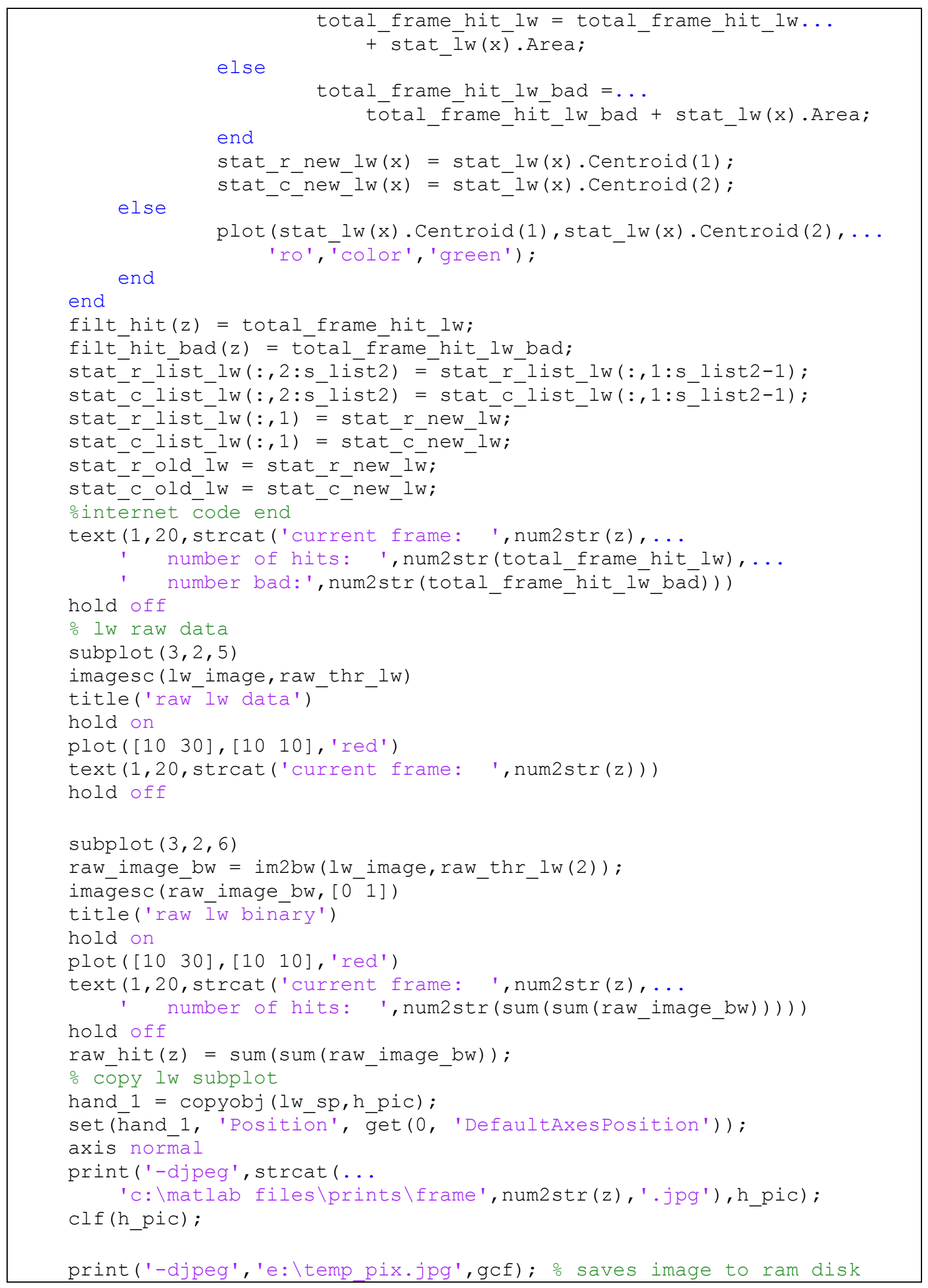

\section{Page 102}




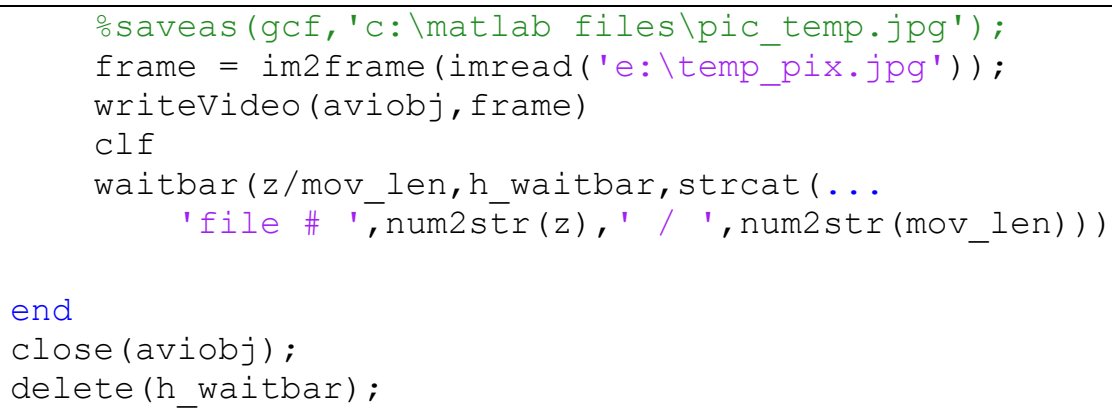

\section{b_iir_filter_test_dual.m:}

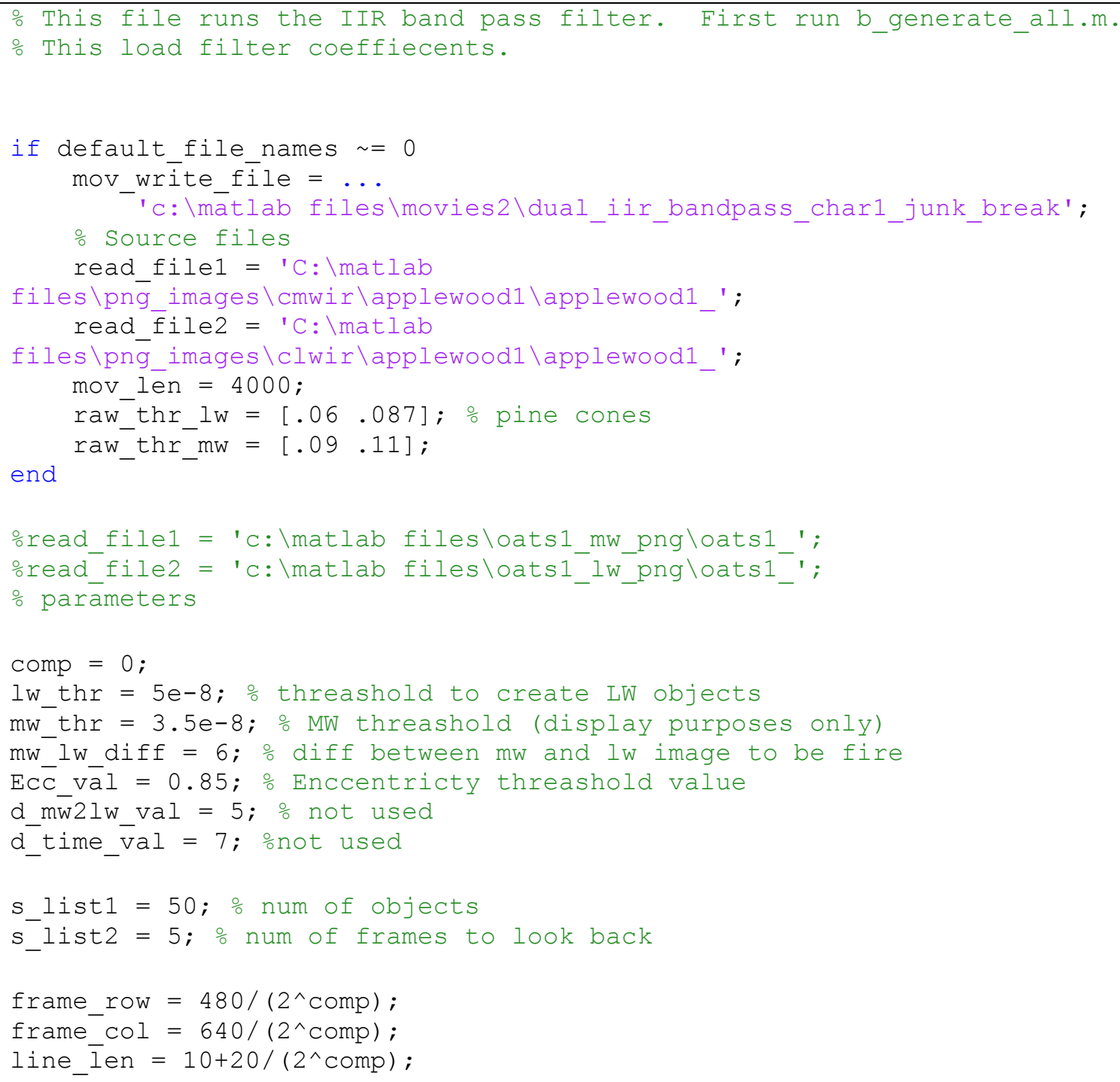

\section{Page 103}




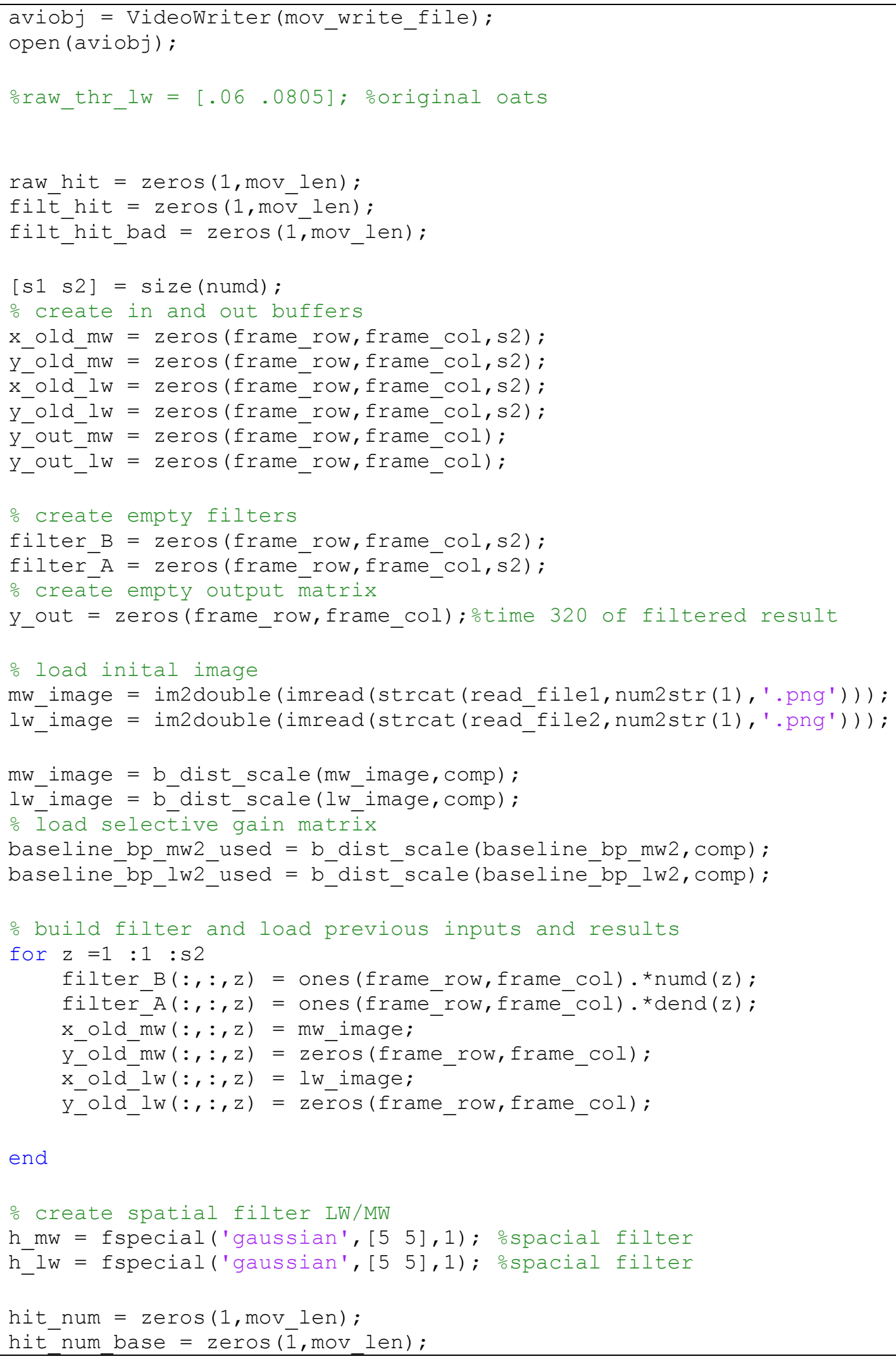

end

\% create spatial filter LW/MW

h_mw = fspecial('gaussian', [5 5],1); ospacial filter

$\mathrm{h}^{-} \mathrm{l} \mathrm{w}=$ fspecial ('gaussian', [5 5],1); \%spacial filter

hit num $=$ zeros $(1$, mov len);

hit num base $=\operatorname{zeros}(\overline{1}, \mathrm{mov} l e n)$;

\section{Page 104}




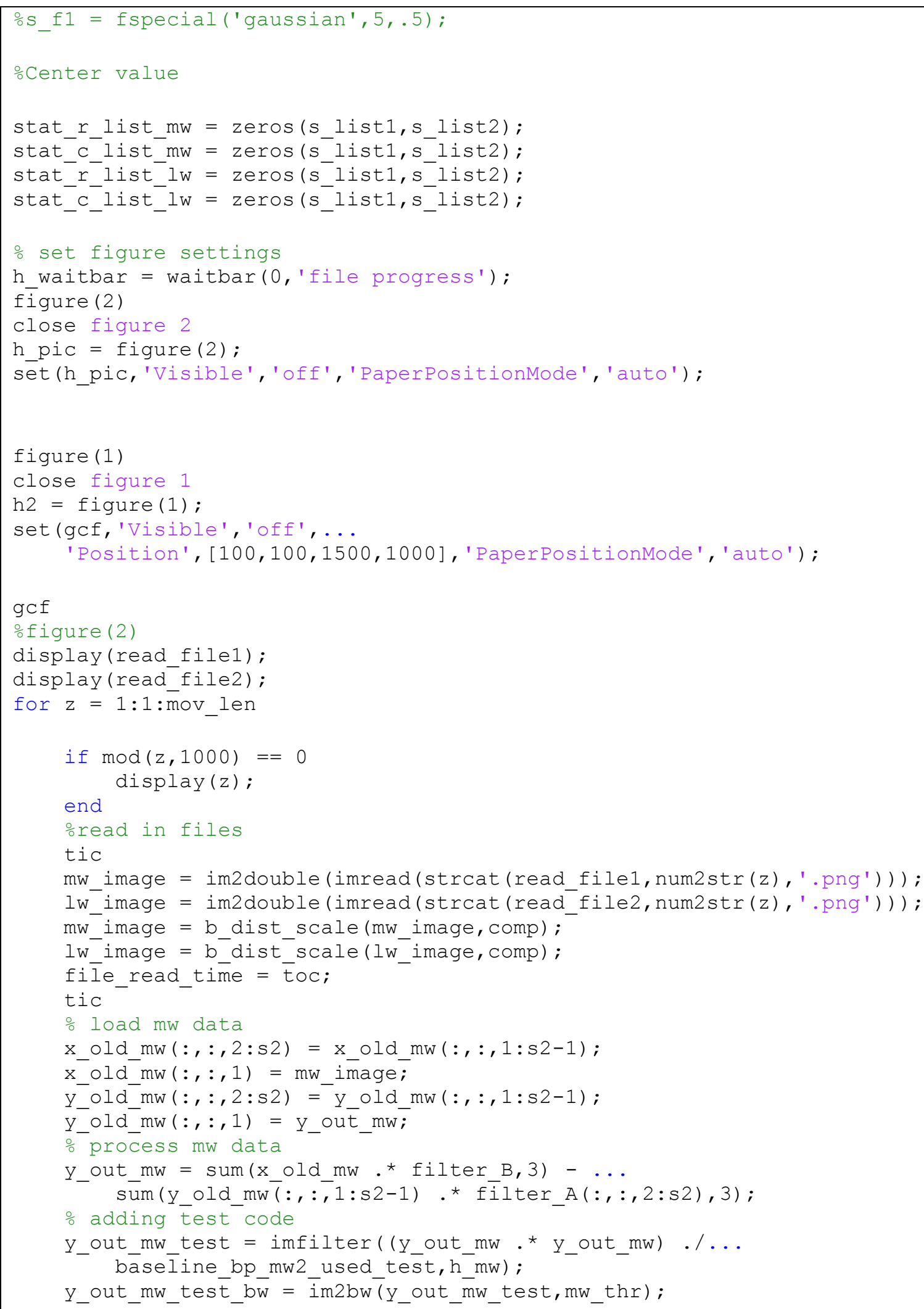

\section{Page 105}




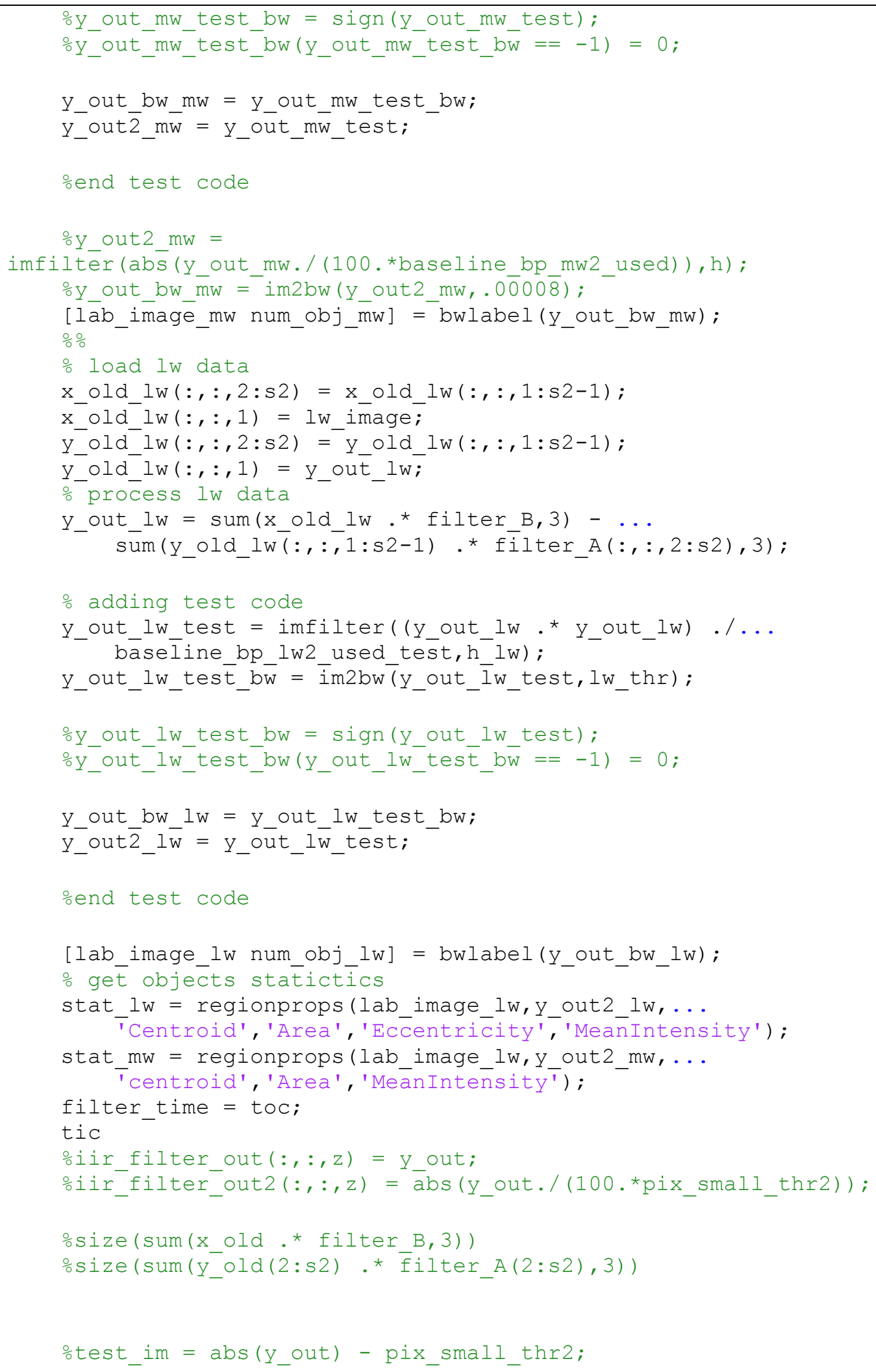




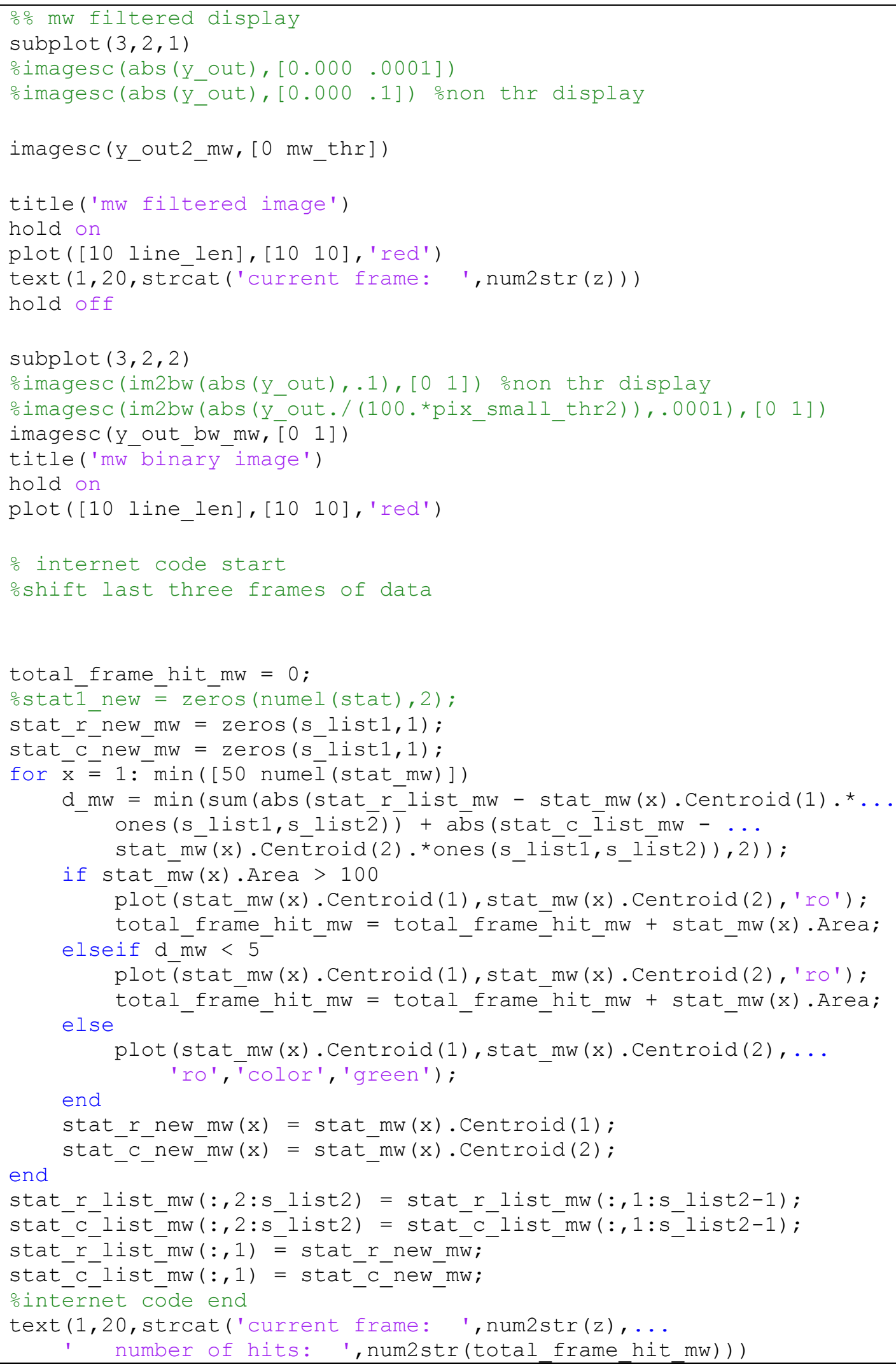




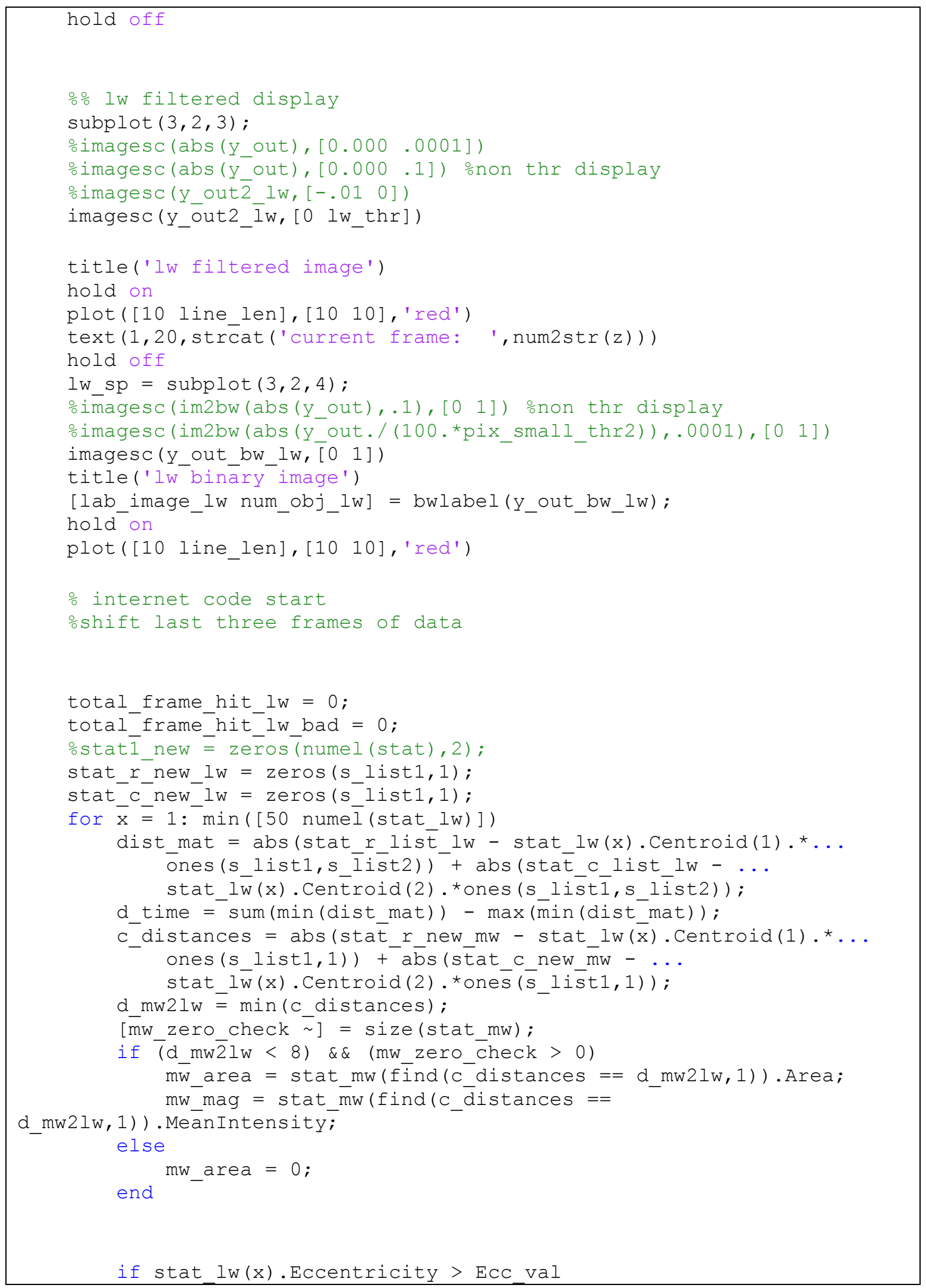

\section{Page 108}




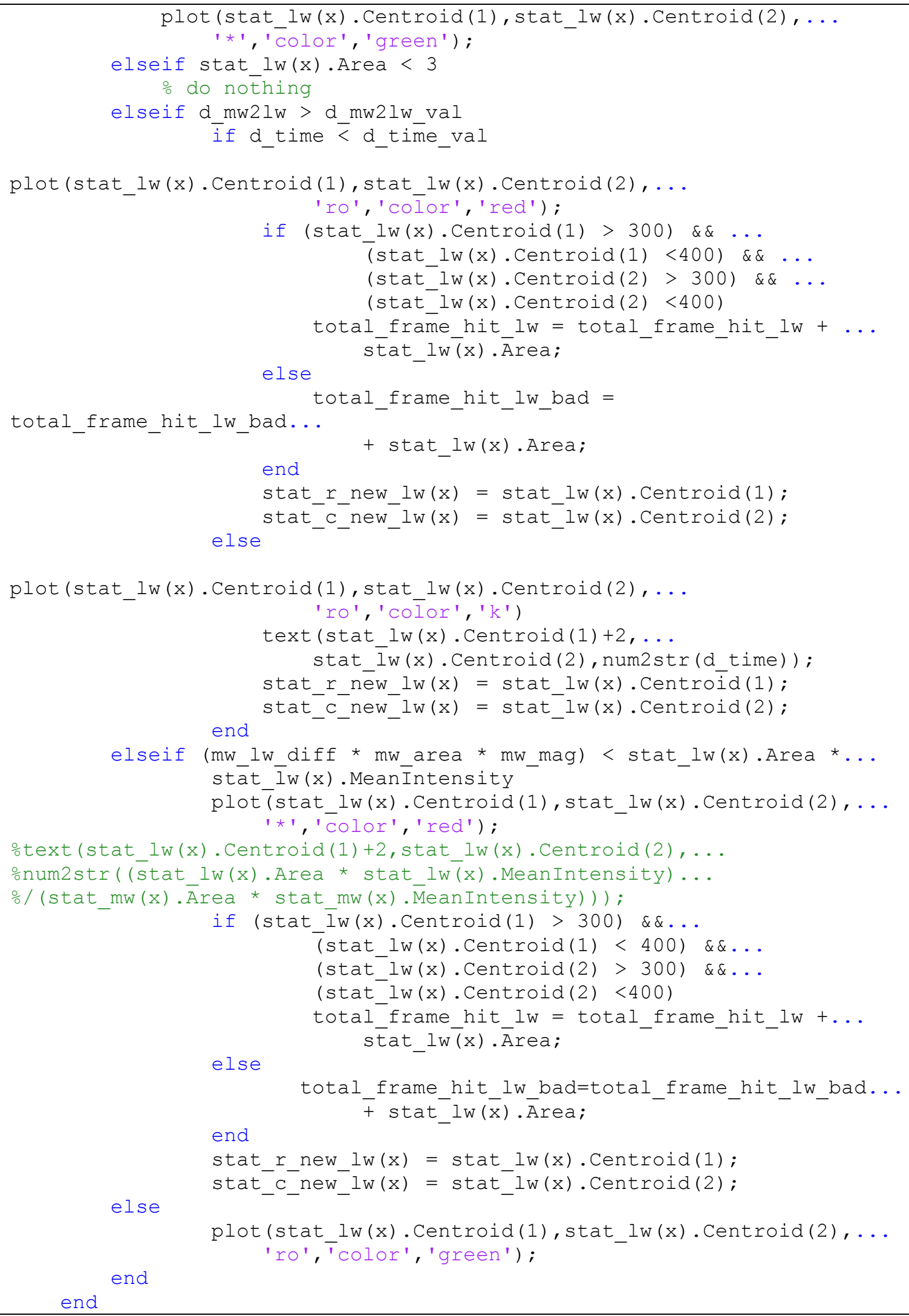

\section{Page 109}




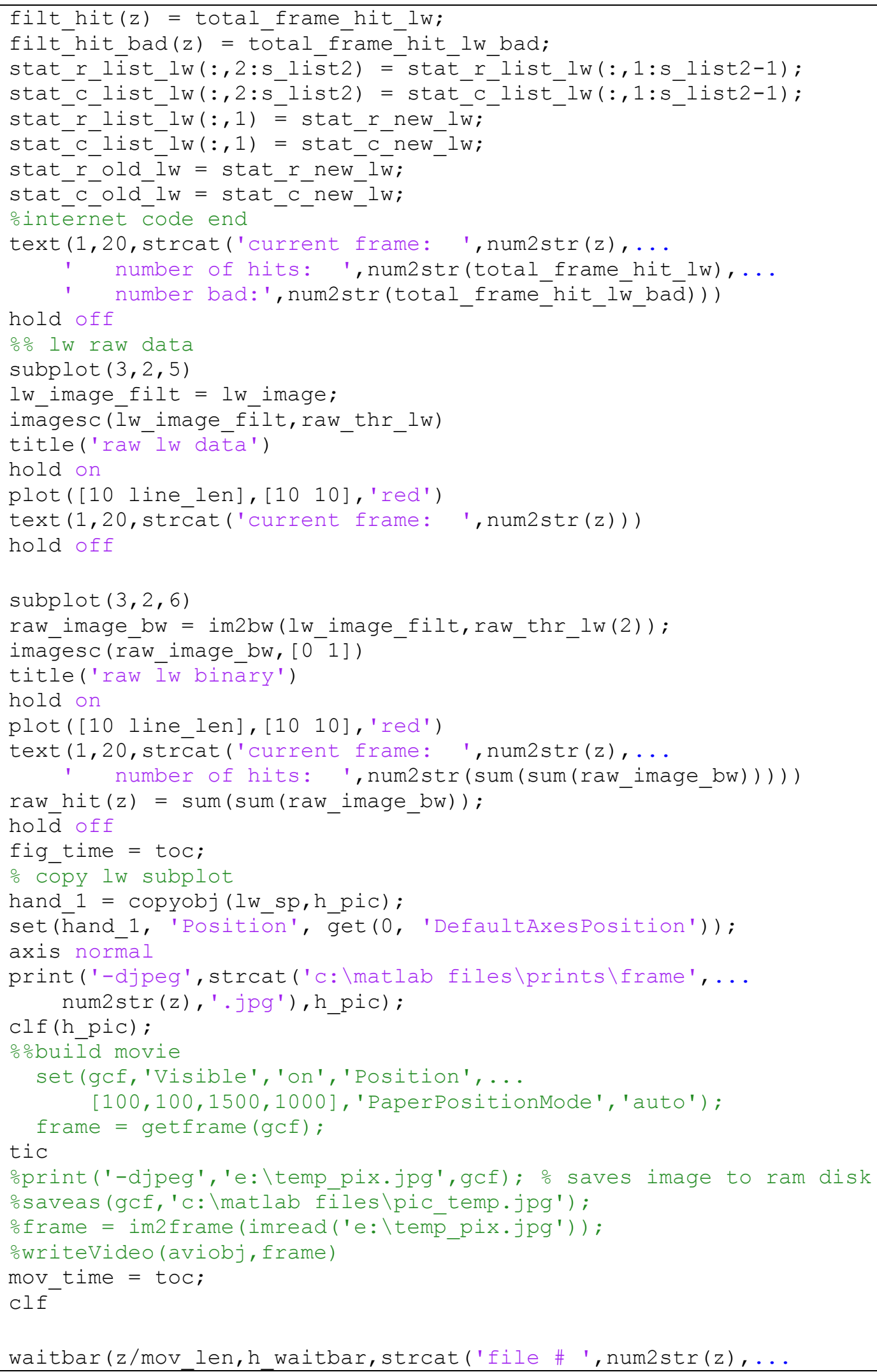

\section{Page 110}




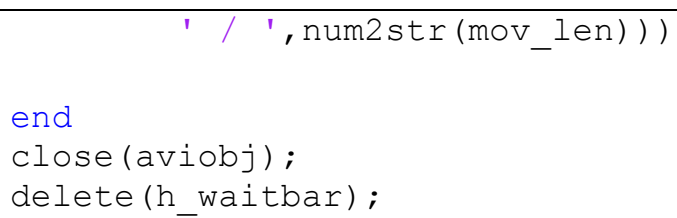

\section{b_batch_movie_gen.m:}

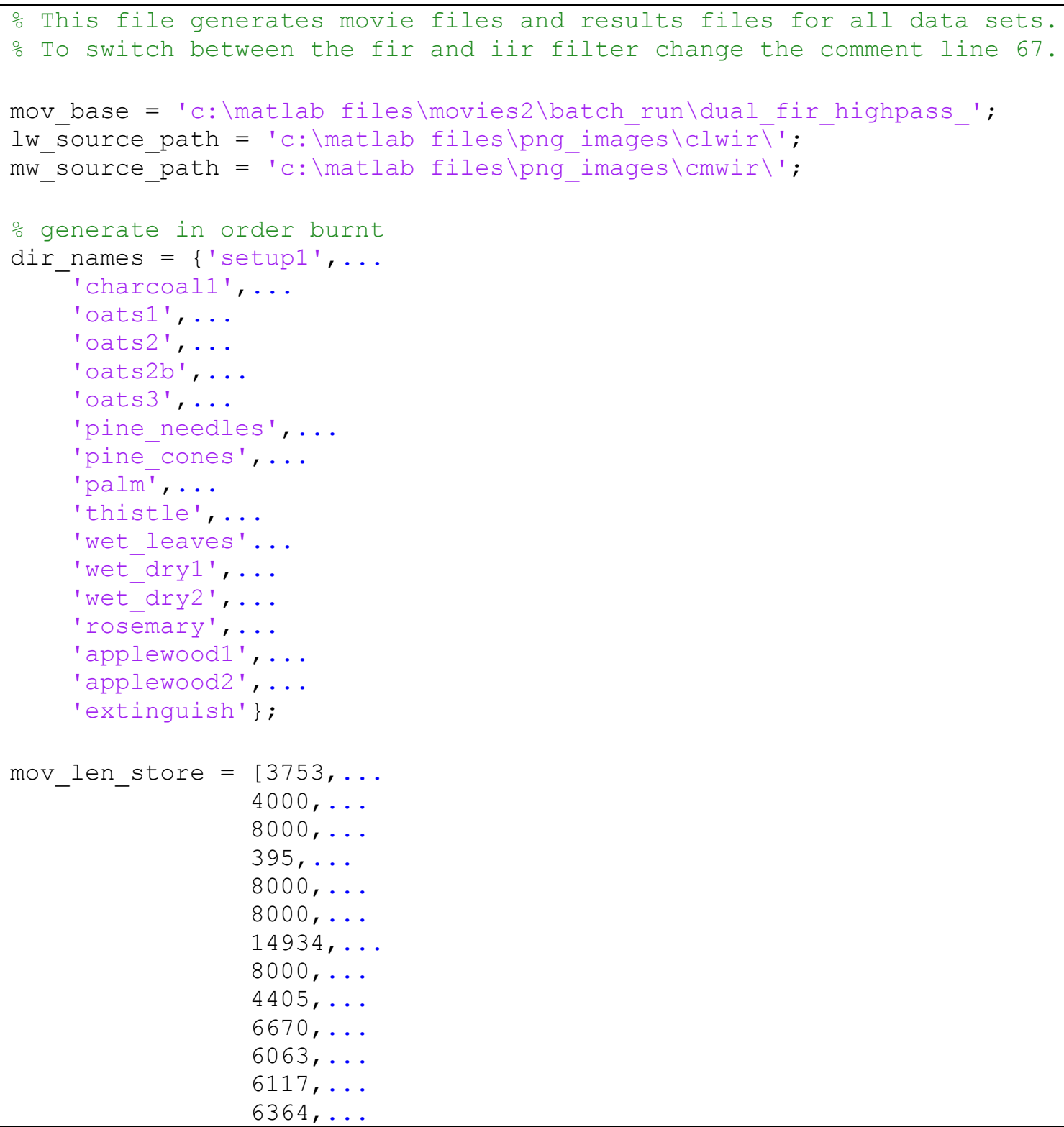




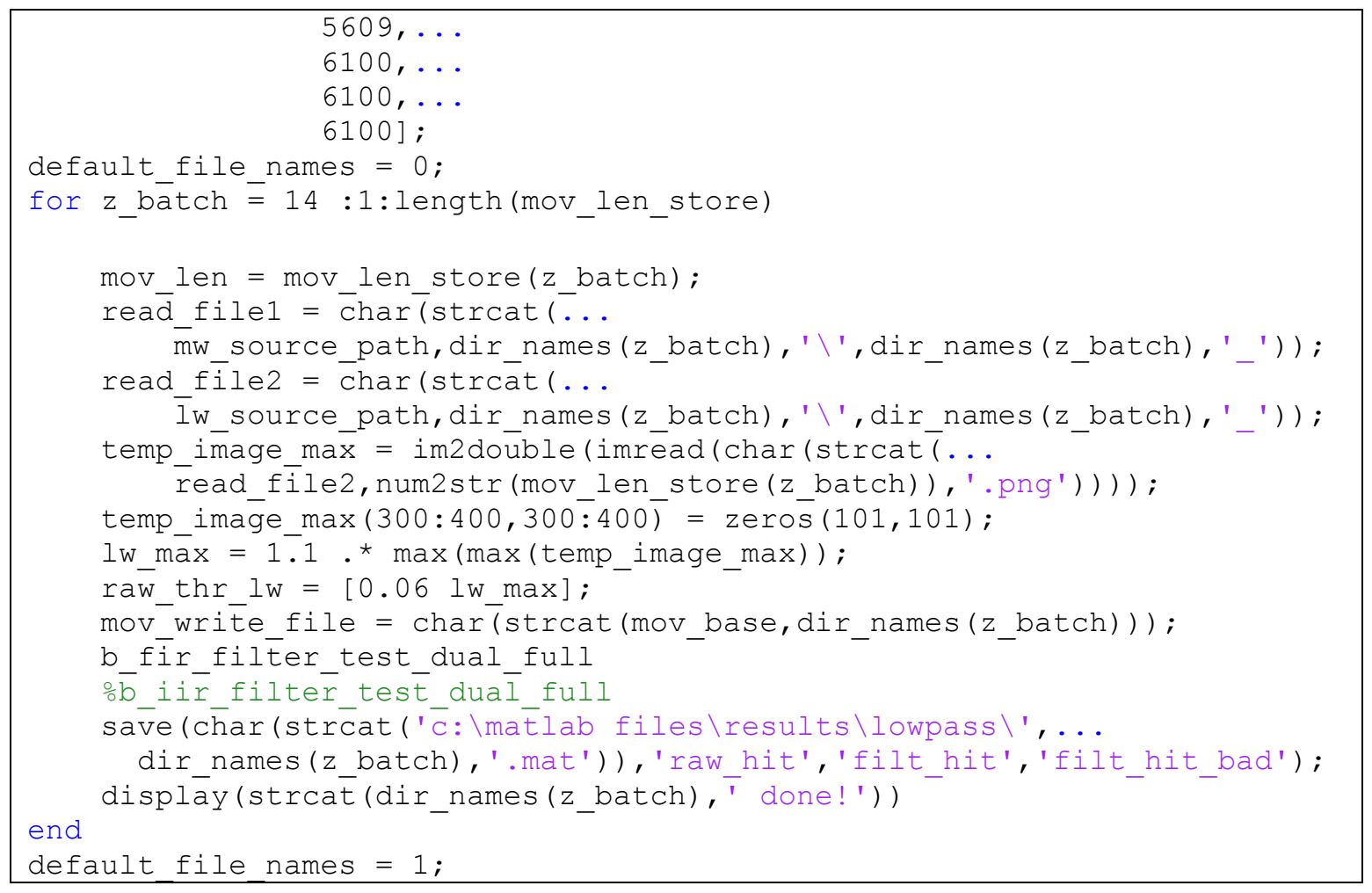

\section{b_results_plots.m:}

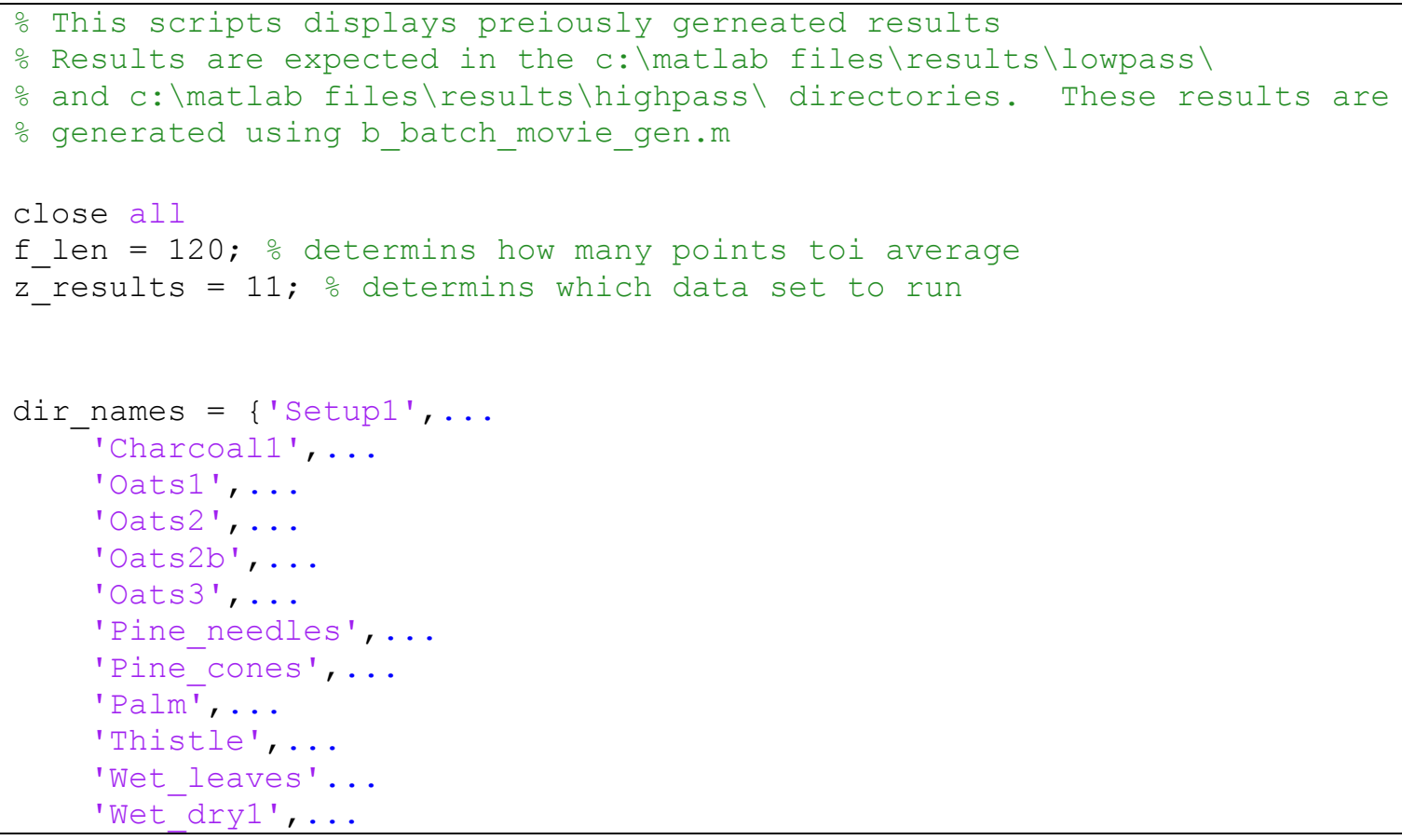




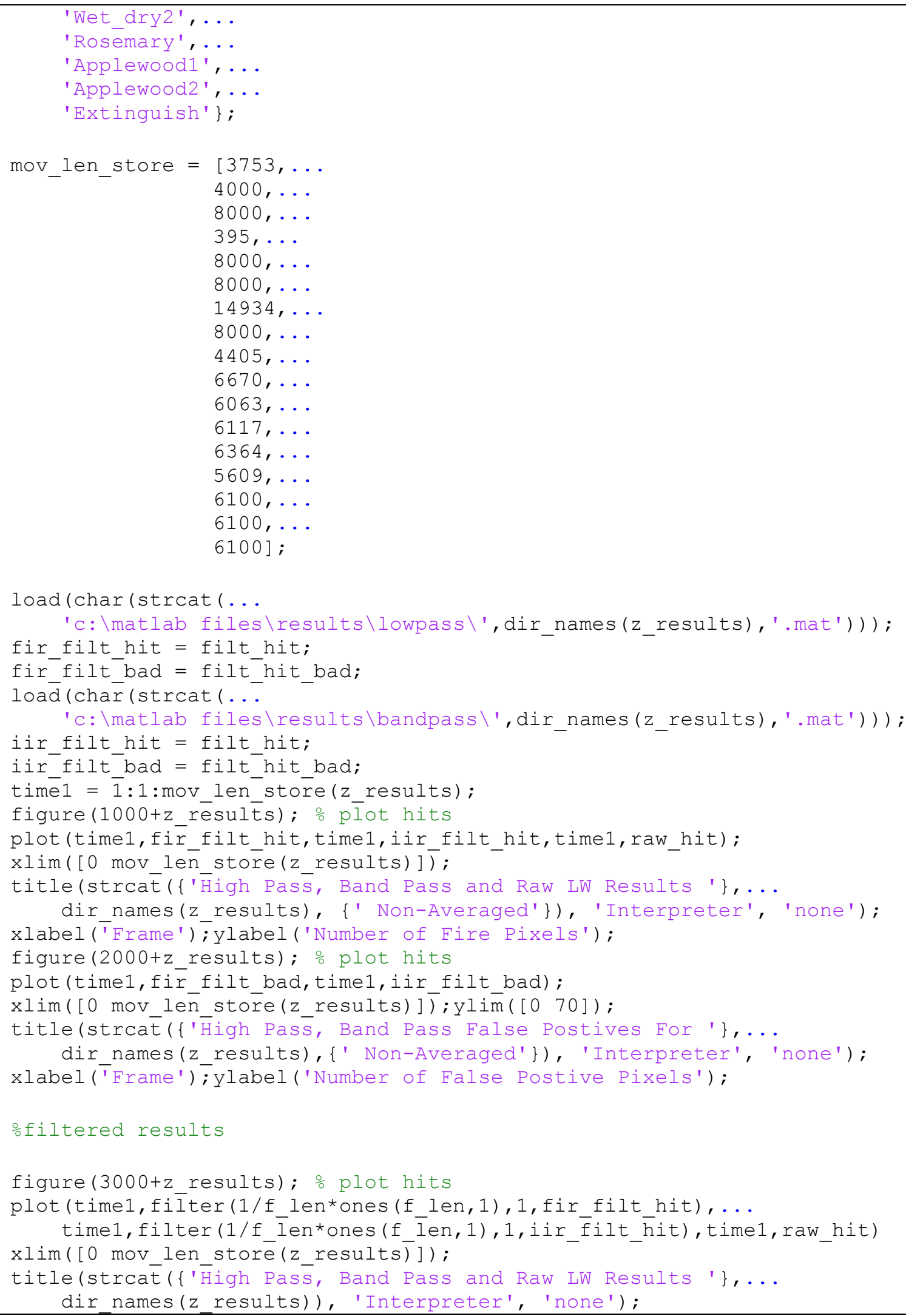

\section{Page 113}


xlabel('Frame'); ylabel('Number of Fire Pixels');

figure $(4000+z$ results); o plot hits

plot(time1, fil̄ter(1/f len*ones (f len,1), 1, fir filt bad),...

time1,filter(1/f_len*ones(f_len,1), 1,iir_filt_bad))

$x \lim \left(\left[0 \mathrm{mov} l e n \_s t o r \bar{e}\left(z \_r e s u l t s\right)\right]\right) ; y \lim \left(\left[\begin{array}{ll}0 & 3 \overline{5}\end{array}\right]\right) ;$

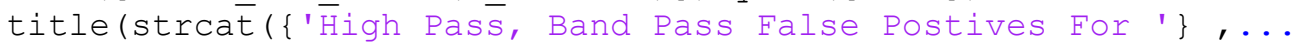
dir_names (z_results)), 'Interpreter', 'none');

xlabel ('Frame');

Page 114 